\title{
Pyridine dipyrrolide bismuth complexes: a transient Bi(II) complex which ring opens cyclic ethers.
}

\author{
Zoë R. Turner*
}

Chemistry Research Laboratory, Department of Chemistry, University of Oxford, Mansfield Road, Oxford, OX1 3TA, United Kingdom. Tel: +44(0) 1865 285157; E-mail: zoe.turner@chem.ox.ac.uk

\section{Table of Contents}

$\begin{array}{lll}\text { I. General considerations } & \text { S2 }\end{array}$

$\begin{array}{lll}\text { II. NMR spectra } & \text { S3 }\end{array}$

$\begin{array}{ll}\text { III. Crystallographic data } & \text { S26 }\end{array}$

$\begin{array}{ll}\text { IV. Electrochemical data } & \text { S70 }\end{array}$

$\begin{array}{lll}\text { V. References } & \text { S71 }\end{array}$ 


\section{General considerations}

All manipulations were carried out using standard Schlenk line or drybox techniques under an atmosphere of dinitrogen or argon. Protio solvents were degassed by sparging with dinitrogen, dried by passing through a column of activated sieves (pentane, hexane, toluene, benzene) and stored over potassium mirrors, or distilled from sodium metal (thf) and stored over activated $4 \AA$ molecular sieves, or distilled from sodium-potassium alloy (diethyl ether) and stored over a potassium mirror. Deuterated solvents were dried over potassium $\left(\mathrm{C}_{6} \mathrm{D}_{6}, \mathrm{C}_{7} \mathrm{D}_{8}\right)$ or $\mathrm{CaH}_{2}\left(\mathrm{C}_{4} \mathrm{D}_{8} \mathrm{O}\right)$, distilled under reduced pressure and freeze-pump-thaw degassed three times prior to use.

${ }^{1} \mathrm{H}$ NMR spectra were recorded at $298 \mathrm{~K}$, unless otherwise stated, on Bruker AVIII 400 nanobay or Bruker AVIII 500 spectrometers and ${ }^{13} \mathrm{C}\left\{{ }^{1} \mathrm{H}\right\}$ or ${ }^{13} \mathrm{C}$ NMR spectra on the same spectrometers at operating frequencies of 100 and $125 \mathrm{MHz}$ respectively. Two dimensional ${ }^{1} \mathrm{H}-{ }^{1} \mathrm{H}$ and ${ }^{13} \mathrm{C}-{ }^{1} \mathrm{H}$ correlation experiments were used, when necessary, to confirm ${ }^{1} \mathrm{H}$ and ${ }^{13} \mathrm{C}$ assignments. All NMR spectra were referenced internally to residual protio solvent $\left({ }^{1} \mathrm{H}\right)$ or solvent $\left({ }^{13} \mathrm{C}\right)$ resonances and are reported relative to tetramethylsilane ( $\delta=0 \mathrm{ppm}$ ). Chemical shifts are quoted in $\delta(\mathrm{ppm})$ and coupling constants in Hertz. Elemental analyses were carried out at London Metropolitan University.

2,6-pyridinedicarboxaldehyde; ${ }^{1}$

2,3,5,6-tetramethyl-1,4-bis(trimethylsilyl)-1,4-diaza2,5-cyclohexadiene (F), 2,5-dimethyl-1,4-bis(trimethylsilyl)-1,4-diaza-2,5-cyclohexadiene (G) or 1,1'-bis(trimethylsilyl)-1,1'-dihydro-4,4'-bipyridine $(\mathbf{H}){ }^{2}$ and $\left[\left({ }^{\mathrm{Mes}} \mathrm{Nacnac}\right) \mathrm{MgMg}\left({ }^{\mathrm{Mes}} \mathrm{Nacnac}\right)\right]^{3}$ (where $\left.{ }^{\text {Mes }} \mathrm{Nacnac}=\left[\left(2,4,6-\mathrm{Mes}-\mathrm{C}_{6} \mathrm{H}_{3}-\mathrm{NCMe}\right)_{2} \mathrm{CH}\right]^{-}\right)$were prepared accorded to reported literature procedures. $\mathrm{BiCl}_{3}$ and $\mathrm{BiBr}_{3}$ were sublimed prior to use. $\mathrm{H}_{2}{ }^{\mathrm{Mes}, \mathrm{Ph}} \mathrm{L}$ was prepared according to a modified literature procedure ${ }^{4-6}$ and has been recently reported by Milsmann et al. in full detail. ${ }^{7}$ Single crystals of $\mathrm{H}_{2}{ }^{\mathrm{Mes}, \mathrm{Ph}} \mathrm{L}$ and $\mathrm{H}_{2}{ }^{\mathrm{Mes}, \mathrm{Ph}} \mathrm{L} \cdot \mathrm{Et}_{2} \mathrm{O}$ were obtained by pentane diffusion into an ethanol solution and from diethyl ether respectively at room temperature. 

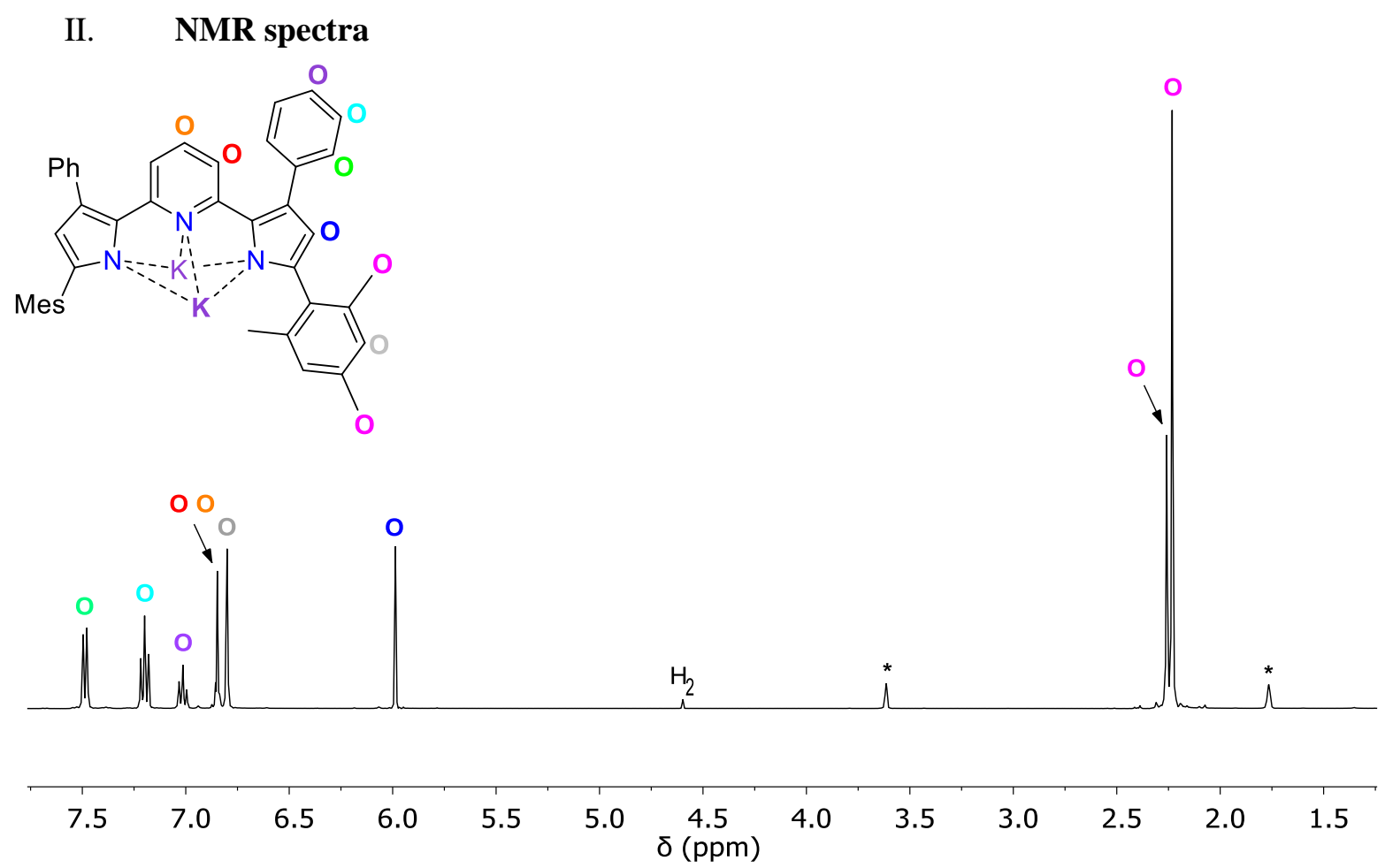

Figure S1: ${ }^{1} \mathrm{H}$ NMR spectrum $\left(\mathrm{C}_{4} \mathrm{D}_{8} \mathrm{O}, 500 \mathrm{MHz}, 298 \mathrm{~K}\right)$ of $\mathrm{K}_{2}{ }^{\mathrm{Mes}, \mathrm{Ph}} \mathrm{L}(\mathbf{1}) . *$ denotes residual protio solvent fraction of $\mathrm{C}_{4} \mathrm{D}_{8} \mathrm{O}$.

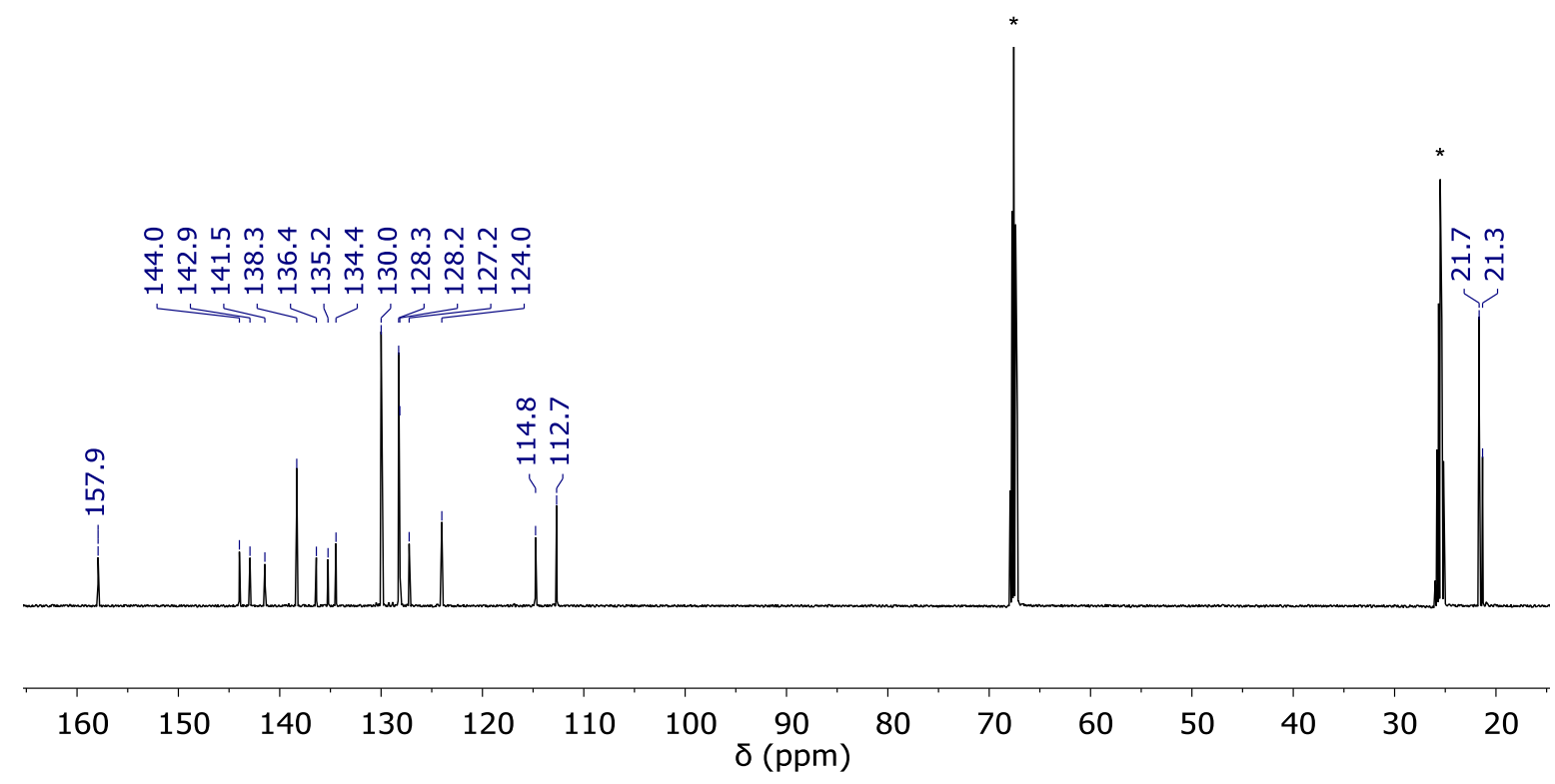

Figure S2: ${ }^{13} \mathrm{C}\left\{{ }^{1} \mathrm{H}\right\}$ NMR spectrum $\left(\mathrm{C}_{4} \mathrm{D}_{8} \mathrm{O}, 125 \mathrm{MHz}, 298 \mathrm{~K}\right)$ of $\mathrm{K}_{2}{ }^{\mathrm{Mes}, \mathrm{Ph}} \mathrm{L}(\mathbf{1}) . *$ denotes $\mathrm{C}_{4} \mathrm{D}_{8} \mathrm{O}$. 

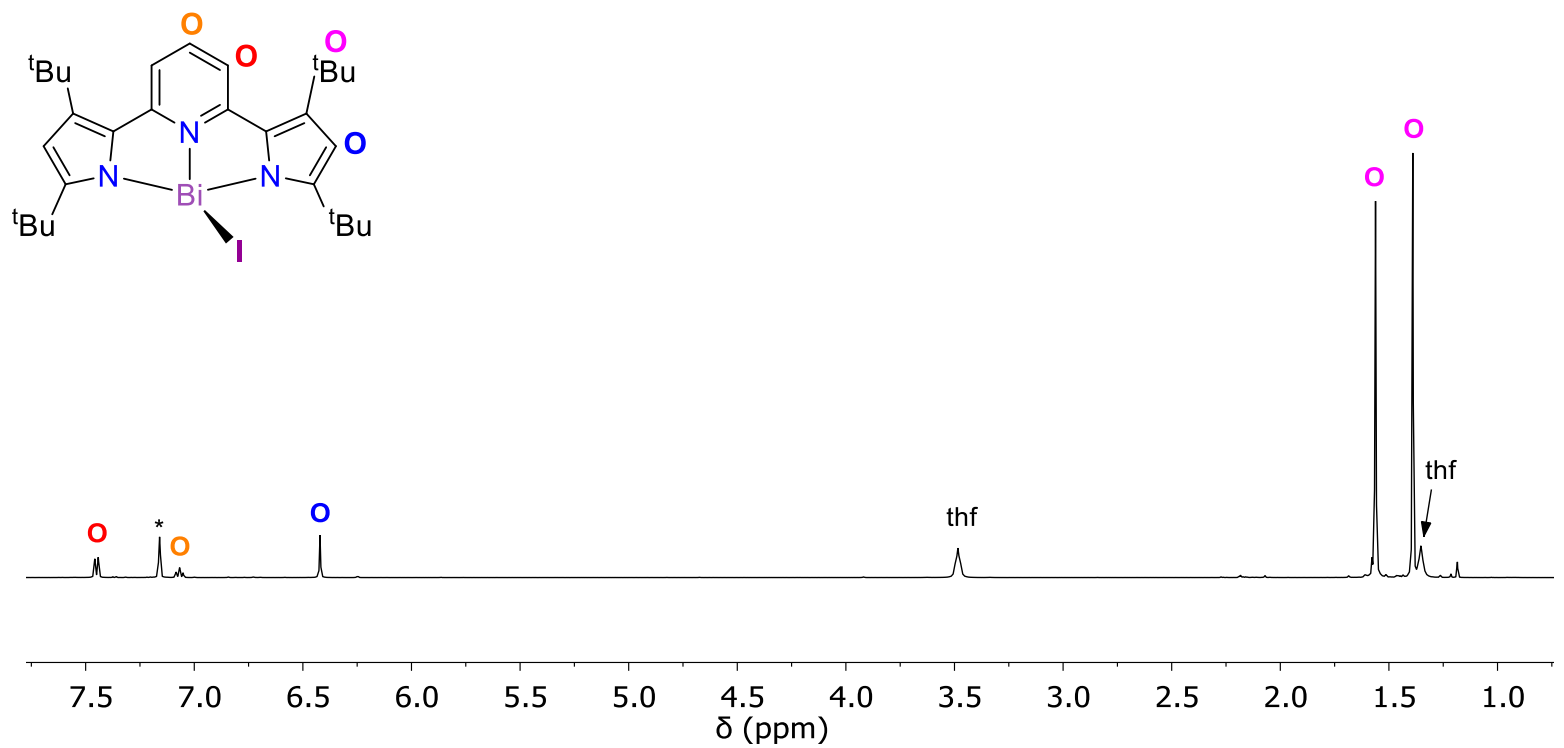

Figure S3: ${ }^{1} \mathrm{H}$ NMR spectrum $\left(\mathrm{C}_{6} \mathrm{D}_{6}, 500 \mathrm{MHz}, 298 \mathrm{~K}\right)$ of $\left({ }^{\mathrm{t}}{ }^{\mathrm{B} u}{ }^{{ }_{\mathrm{B} u}} \mathrm{~L}\right) \mathrm{BiI}(\mathbf{2}) . *$ denotes residual protio solvent fraction of $\mathrm{C}_{6} \mathrm{D}_{6}$.

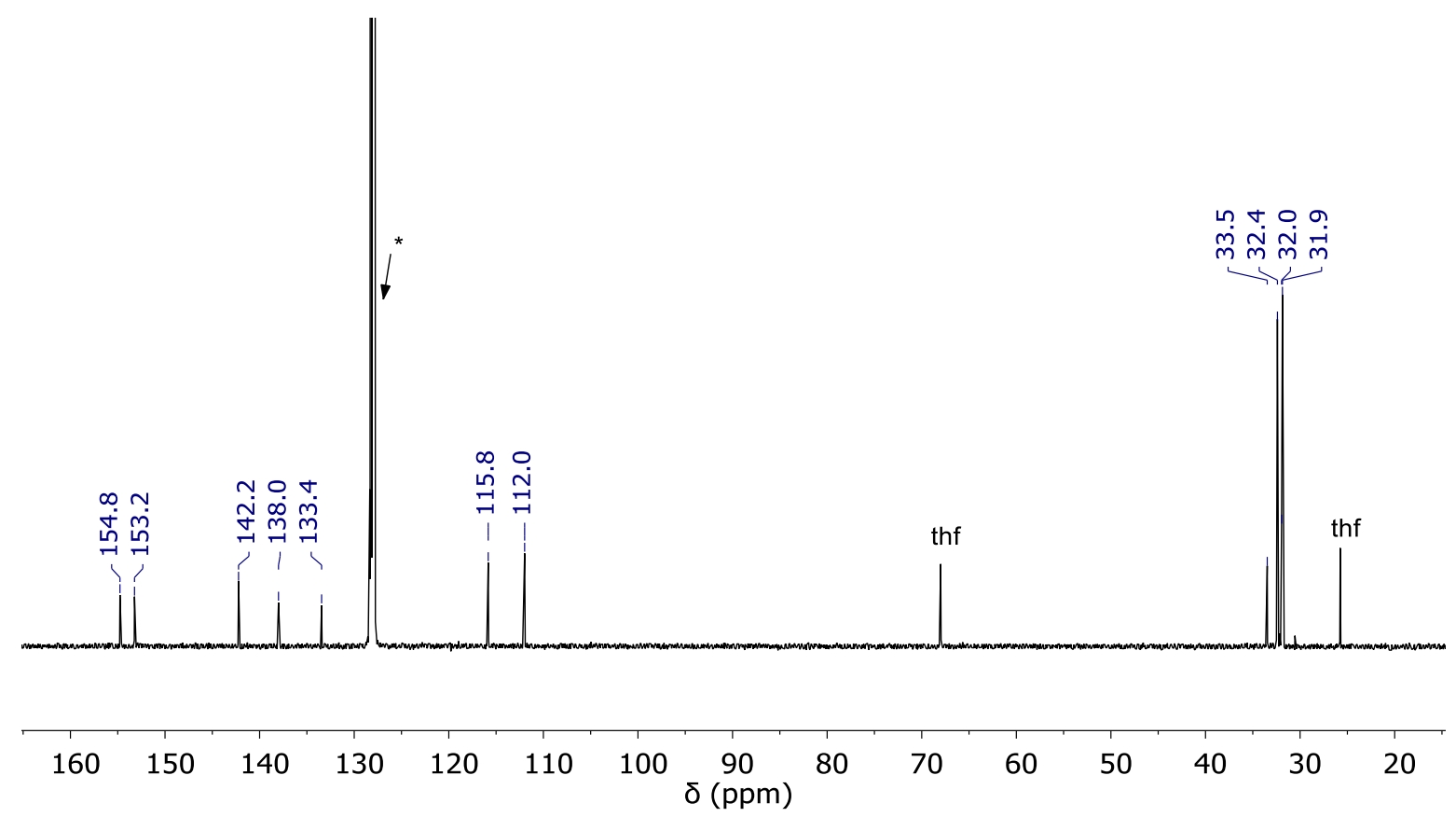

Figure S4: ${ }^{13} \mathrm{C}\left\{{ }^{1} \mathrm{H}\right\}$ NMR spectrum $\left(\mathrm{C}_{6} \mathrm{D}_{6}, 125 \mathrm{MHz}, 298 \mathrm{~K}\right)$ of $\left({ }^{\mathrm{t} B u}{ }^{\mathrm{t}}{ }^{\mathrm{Bu}} \mathrm{L}\right) \mathrm{BiI}(\mathbf{2}) . *$ denotes $\mathrm{C}_{6} \mathrm{D}_{6}$. 

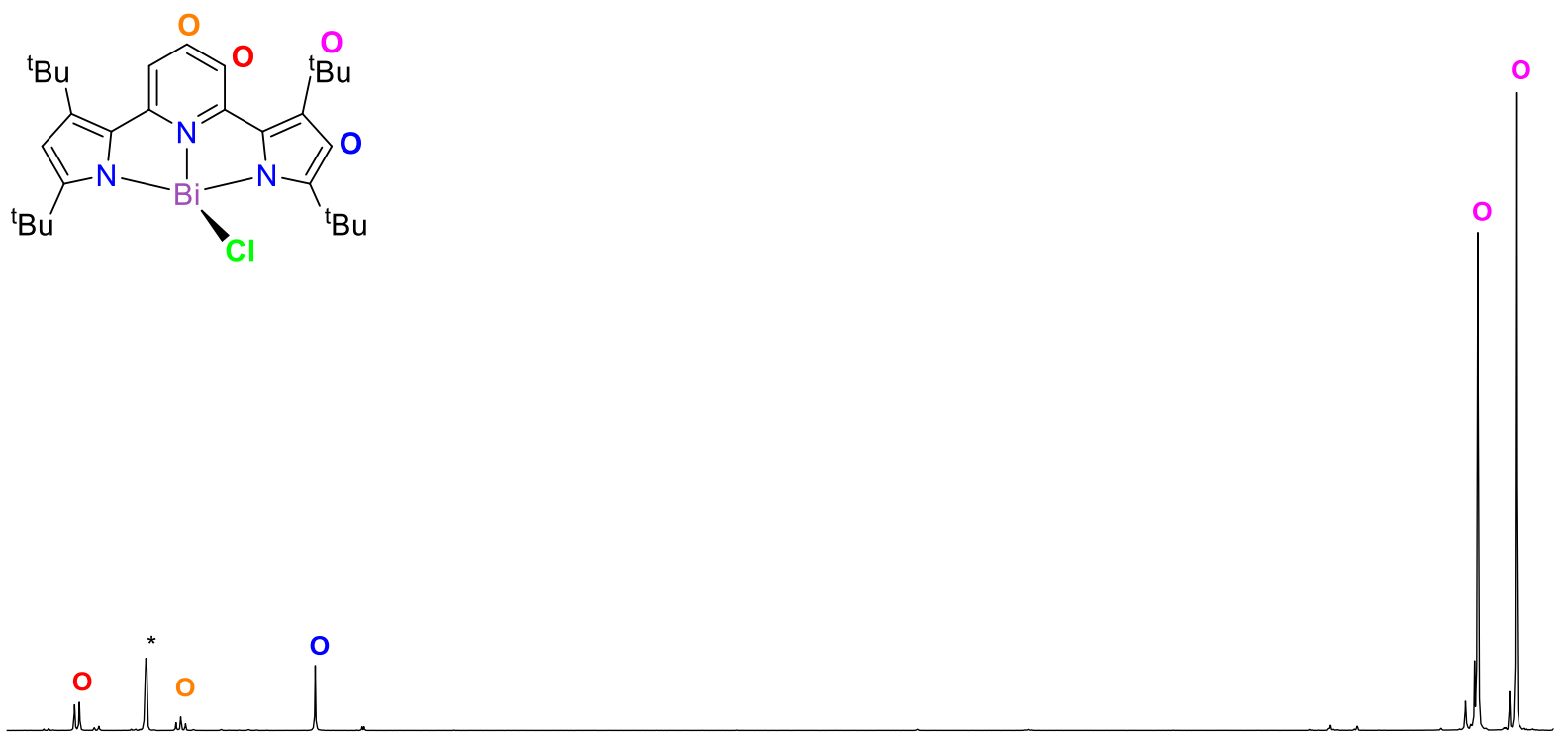

\begin{tabular}{lllllllllllll}
\hline 7.5 & 7.0 & 6.5 & 6.0 & 5.5 & 5.0 & $\begin{array}{c}4.5 \\
\delta(\mathrm{ppm})\end{array}$ & 4.0 & 3.5 & 3.0 & 2.5 & 2.0 & 1.5
\end{tabular}

Figure S5: ${ }^{1} \mathrm{H}$ NMR spectrum $\left(\mathrm{C}_{6} \mathrm{D}_{6}, 500 \mathrm{MHz}, 298 \mathrm{~K}\right)$ of $\left({ }^{\mathrm{t} B u},{ }^{\mathrm{t}} \mathrm{Bu}\right) \mathrm{BiCl}(\mathbf{3}) . *$ denotes residual protio solvent fraction of $\mathrm{C}_{6} \mathrm{D}_{6}$.

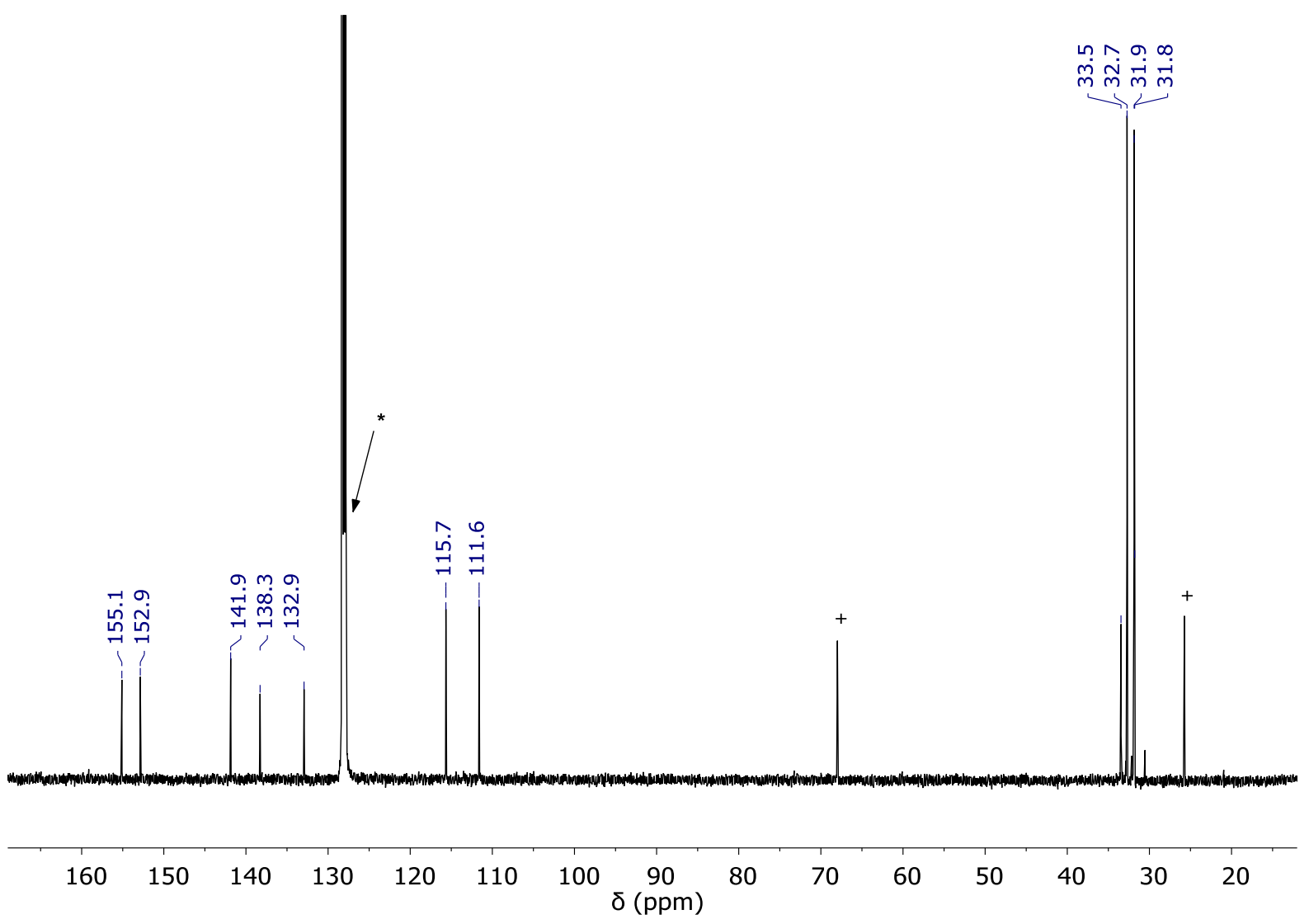

Figure S6: ${ }^{13} \mathrm{C}\left\{{ }^{1} \mathrm{H}\right\}$ NMR spectrum $\left(\mathrm{C}_{6} \mathrm{D}_{6}, 125 \mathrm{MHz}, 298 \mathrm{~K}\right)$ of $\left({ }^{\mathrm{t} B u}{ }^{\mathrm{B}}{ }^{\mathrm{Bu}} \mathrm{L}\right) \mathrm{BiCl}(\mathbf{3})$. + denotes free thf. * denotes $\mathrm{C}_{6} \mathrm{D}_{6}$. 


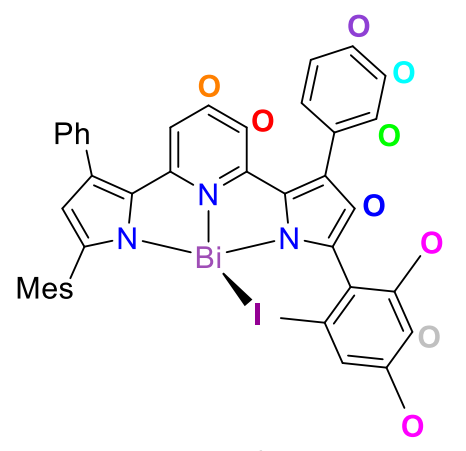

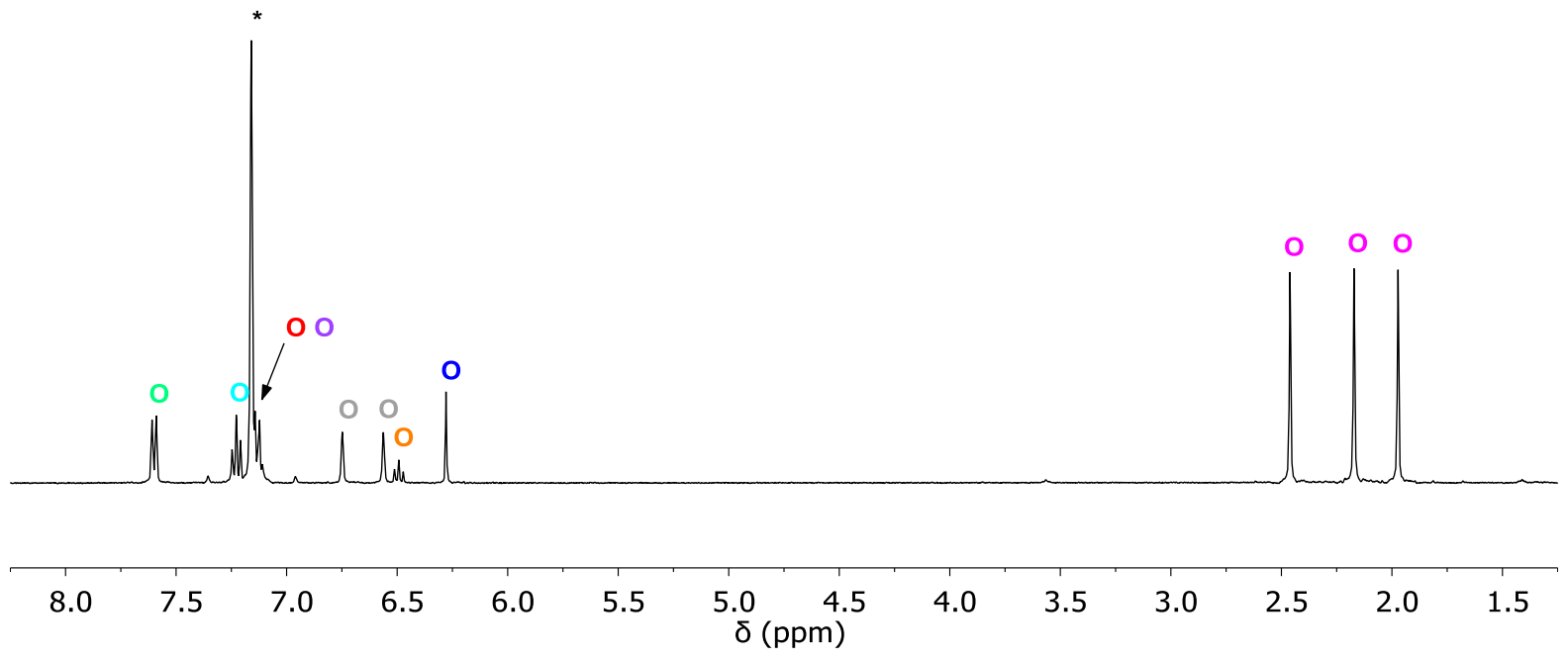

Figure S7: ${ }^{1} \mathrm{H}$ NMR spectrum $\left(\mathrm{C}_{6} \mathrm{D}_{6}, 400 \mathrm{MHz}, 298 \mathrm{~K}\right)$ of $\left({ }^{\mathrm{Mes}, \mathrm{Ph}} \mathrm{L}\right) \mathrm{BiI}(\mathbf{4}) . *$ denotes residual protio solvent fraction of $\mathrm{C}_{6} \mathrm{D}_{6}$.

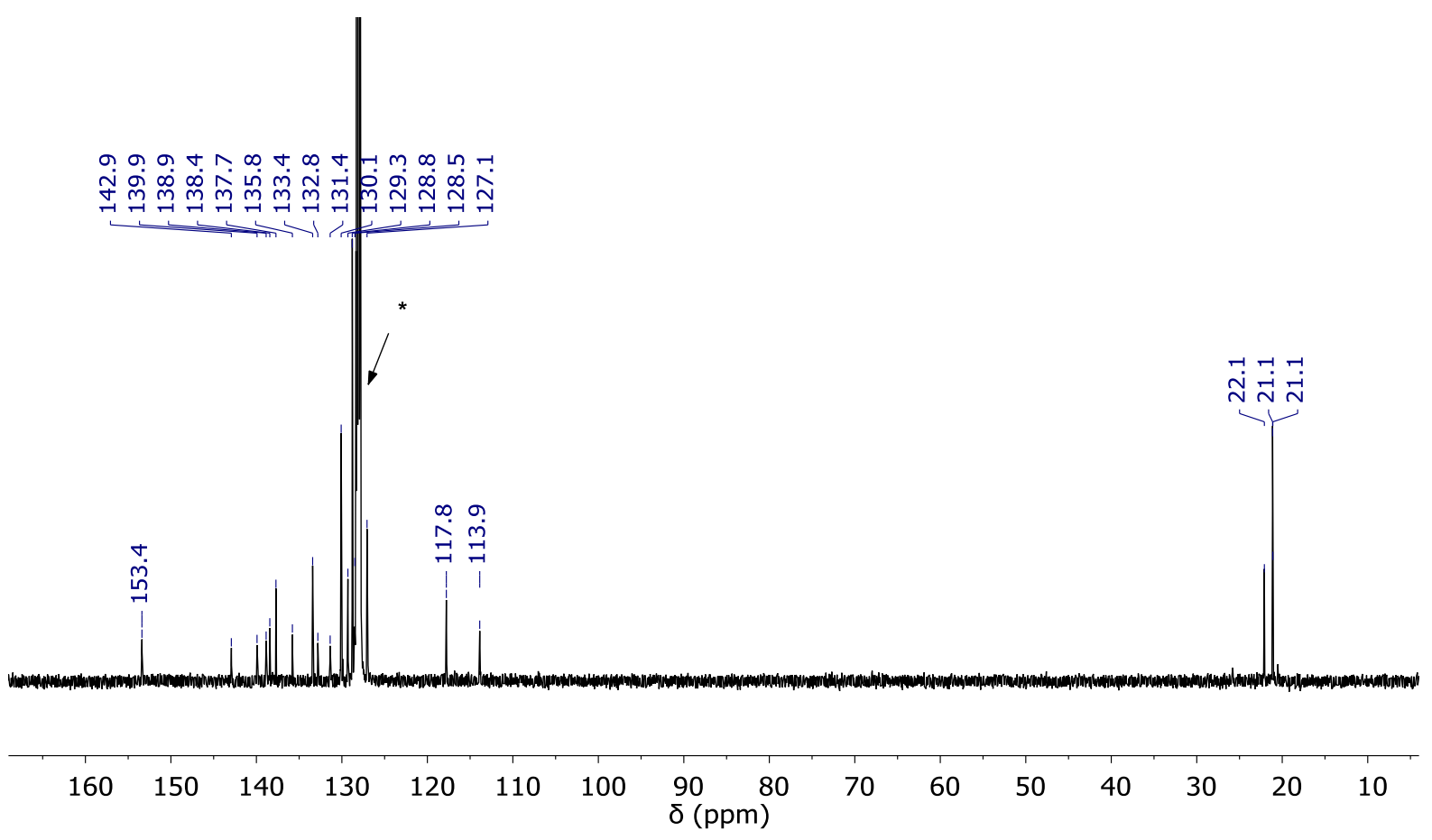

Figure S8: ${ }^{13} \mathrm{C}\left\{{ }^{1} \mathrm{H}\right\}$ NMR spectrum $\left(\mathrm{C}_{6} \mathrm{D}_{6}, 125 \mathrm{MHz}, 298 \mathrm{~K}\right)$ of $\left({ }^{\mathrm{Mes}, P h} \mathrm{~L}\right) \mathrm{BiI}(\mathbf{4})$. + denotes free thf. $*$ denotes $\mathrm{C}_{6} \mathrm{D}_{6}$. 


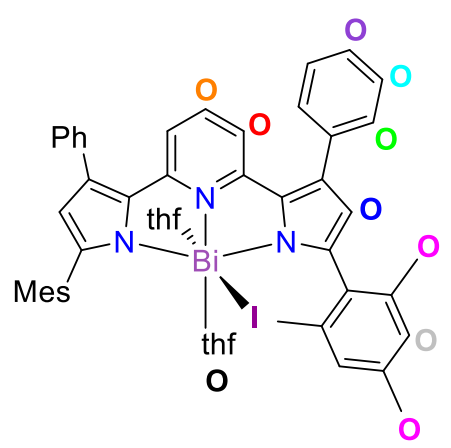

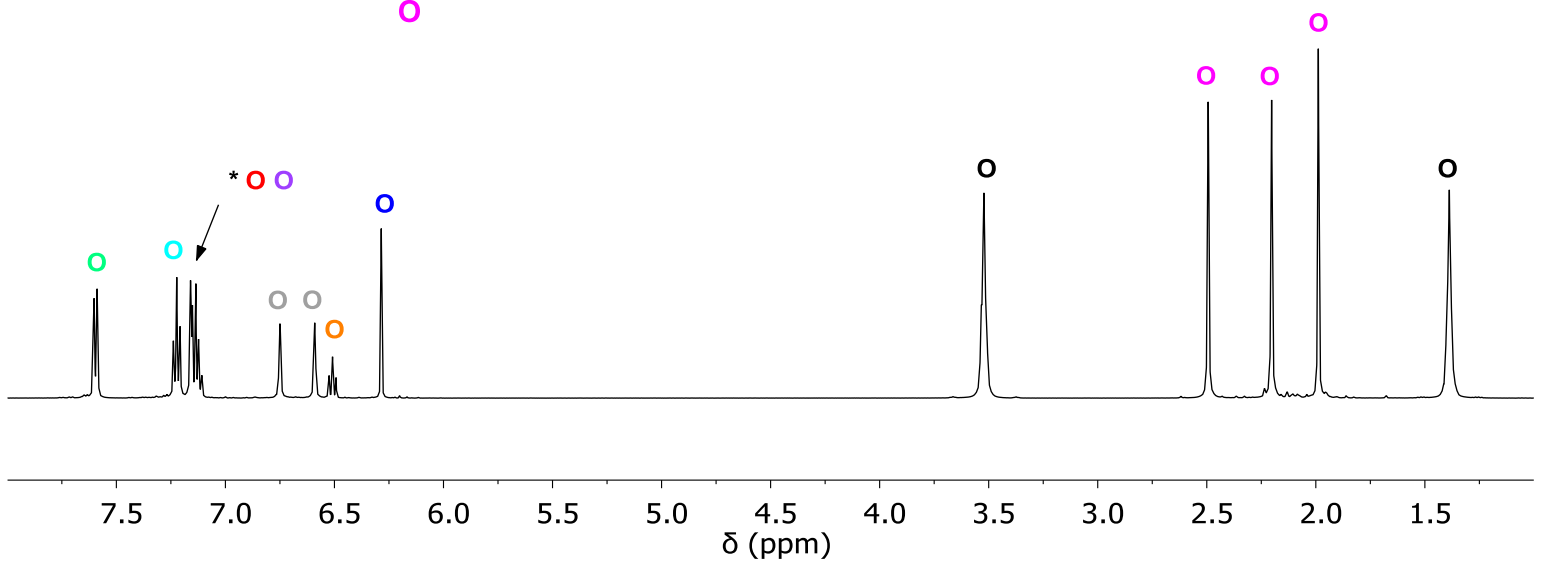

Figure S9: ${ }^{1} \mathrm{H}$ NMR spectrum $\left(\mathrm{C}_{6} \mathrm{D}_{6}, 500 \mathrm{MHz}, 298 \mathrm{~K}\right)$ of $\left({ }^{\mathrm{Mes}, \mathrm{Ph}} \mathrm{L}\right) \mathrm{BiI}(\mathrm{thf})_{2}\left(\mathbf{4} \cdot\right.$ thf $\left.\mathbf{f}_{2}\right) . *$ denotes residual protio solvent fraction of $\mathrm{C}_{6} \mathrm{D}_{6}$.

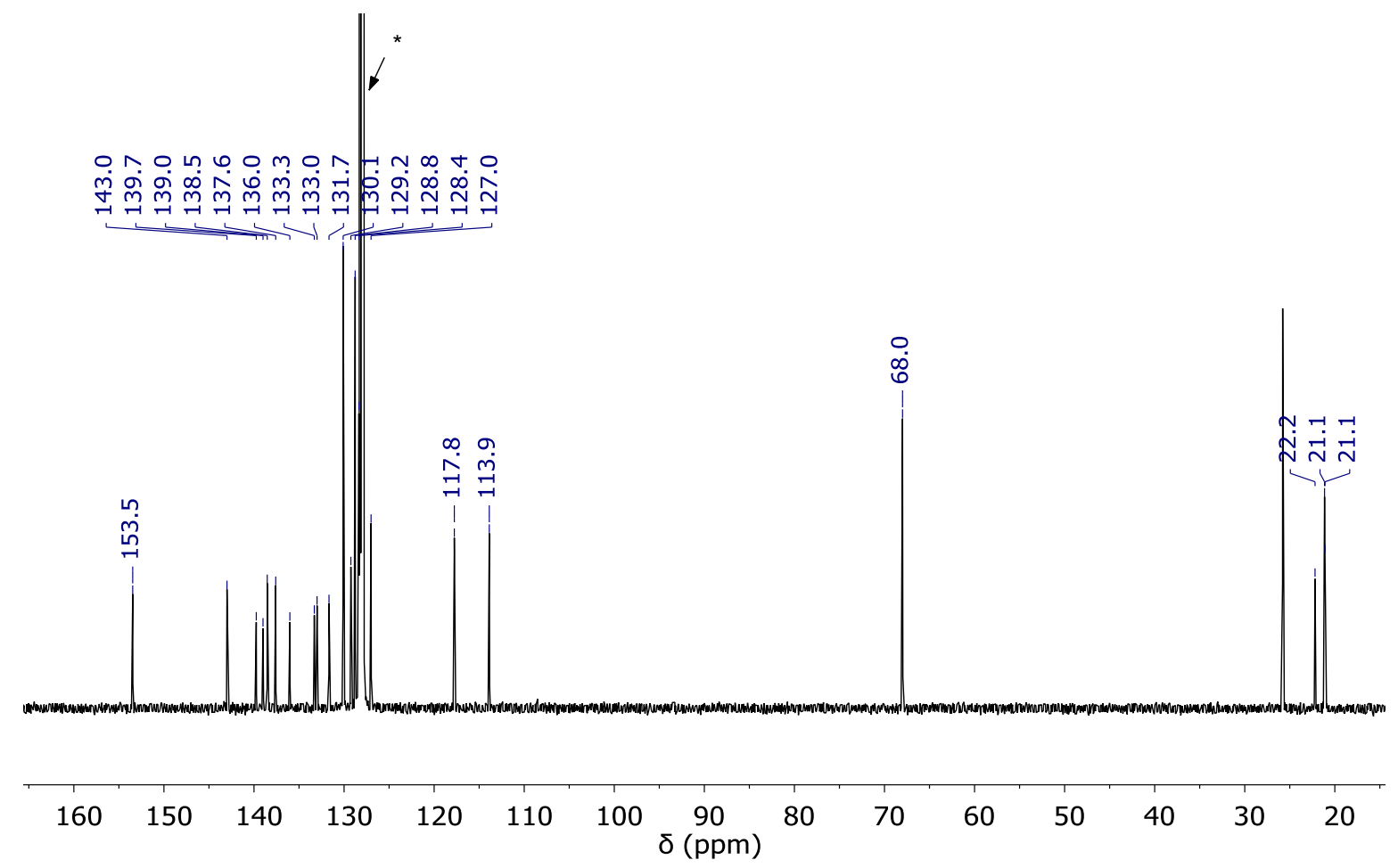

Figure S10: ${ }^{13} \mathrm{C}\left\{{ }^{1} \mathrm{H}\right\}$ NMR spectrum $\left(\mathrm{C}_{6} \mathrm{D}_{6}, 125 \mathrm{MHz}, 298 \mathrm{~K}\right)$ of $\left({ }^{\mathrm{Mes}, \mathrm{Ph}} \mathrm{L}\right) \mathrm{BiI}(\text { thf })_{2}$ (4- thf 2$)$. $*$ denotes $\mathrm{C}_{6} \mathrm{D}_{6}$. 


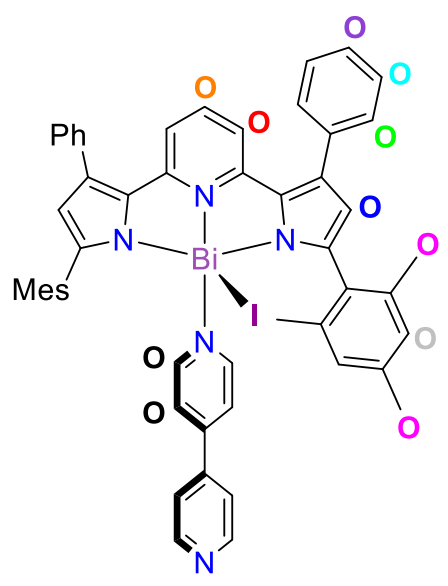

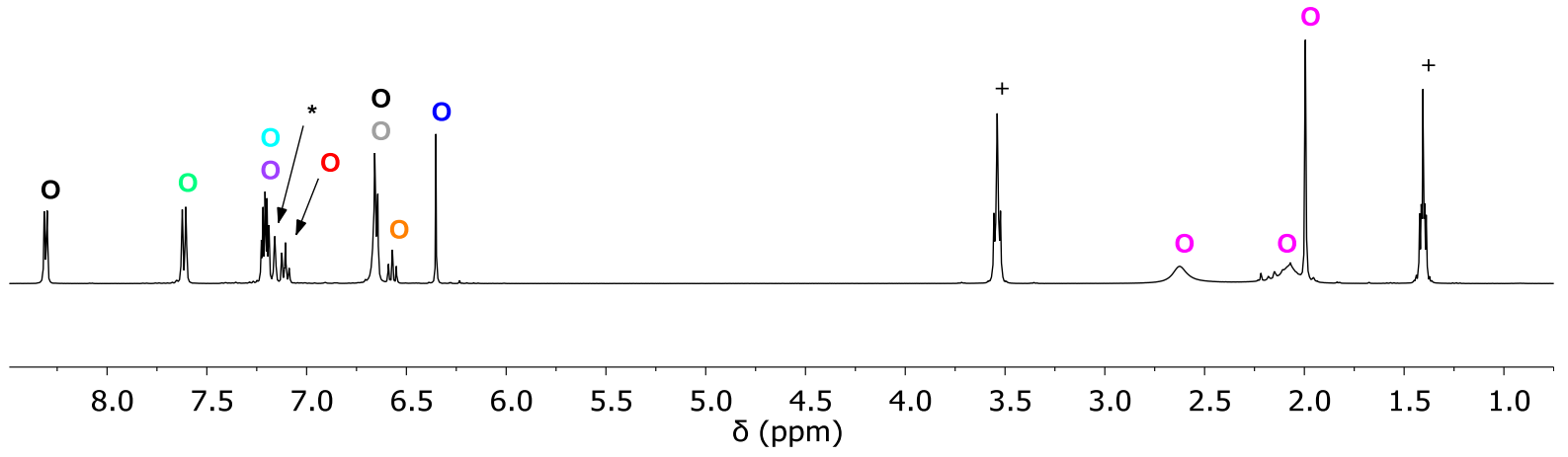

Figure S11: ${ }^{1} \mathrm{H}$ NMR spectrum $\left(\mathrm{C}_{6} \mathrm{D}_{6}, 500 \mathrm{MHz}, 298 \mathrm{~K}\right)$ of $\left({ }^{\mathrm{Mes}, \mathrm{Ph}} \mathrm{L}\right) \mathrm{BiI}(4,4$ '-bipy) $(\mathbf{4} \cdot \mathbf{4 , 4}$ '-bipy $) .+$ denotes thf. $*$ denotes residual protio solvent fraction of $\mathrm{C}_{6} \mathrm{D}_{6}$.

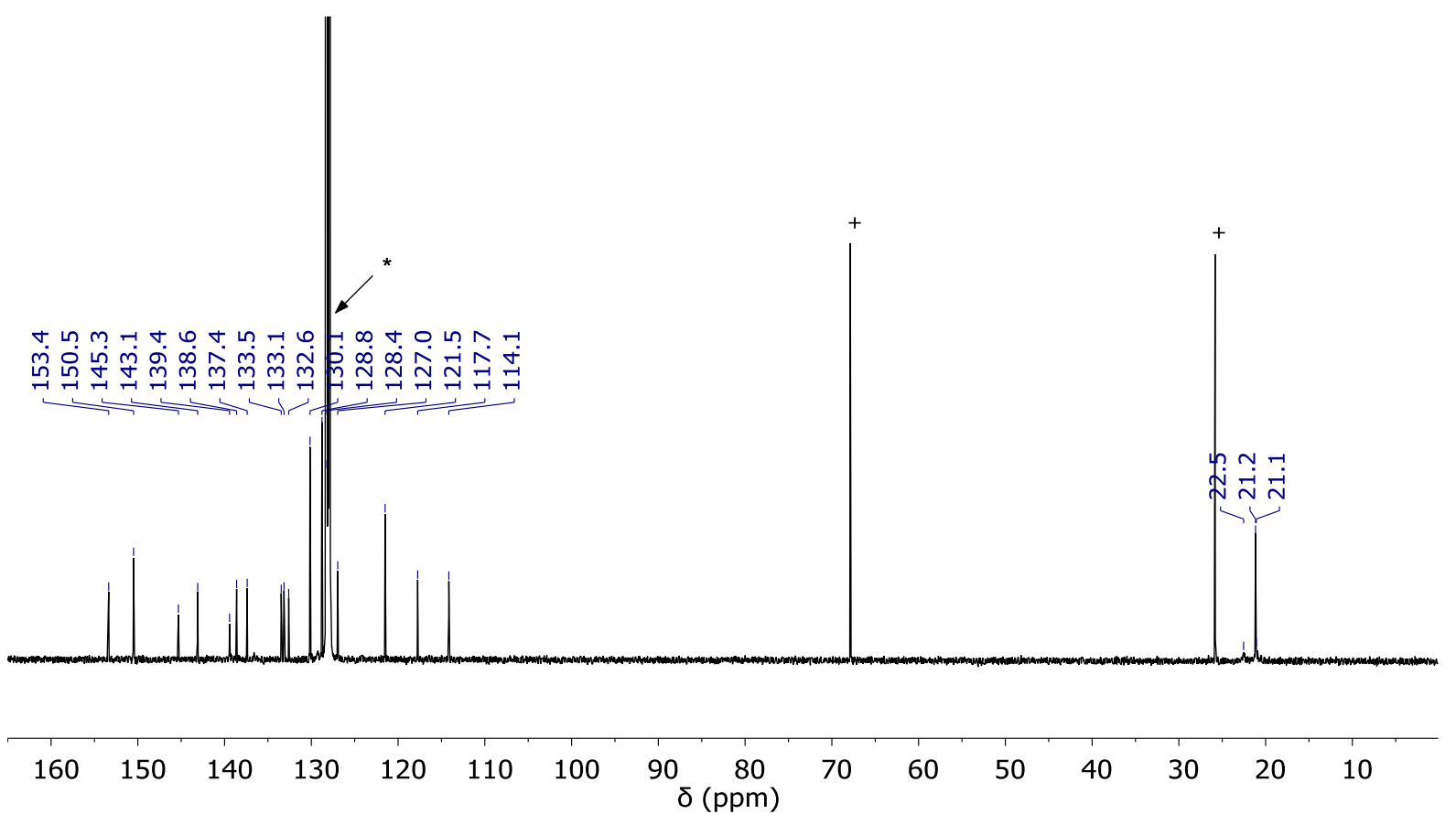

Figure S12: ${ }^{13} \mathrm{C}\left\{{ }^{1} \mathrm{H}\right\}$ NMR spectrum $\left(\mathrm{C}_{6} \mathrm{D}_{6}, 125 \mathrm{MHz}, 298 \mathrm{~K}\right)$ of $\left({ }^{\mathrm{Mes}, \mathrm{Ph}} \mathrm{L}\right) \mathrm{BiI}(4,4$ '-bipy $)(\mathbf{4} \cdot \mathbf{4 , 4}$ '-bipy $)$. + denotes thf. $*$ denotes $\mathrm{C}_{6} \mathrm{D}_{6}$. 


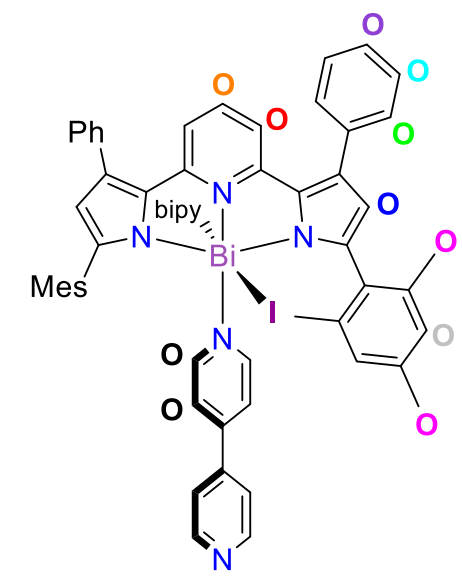
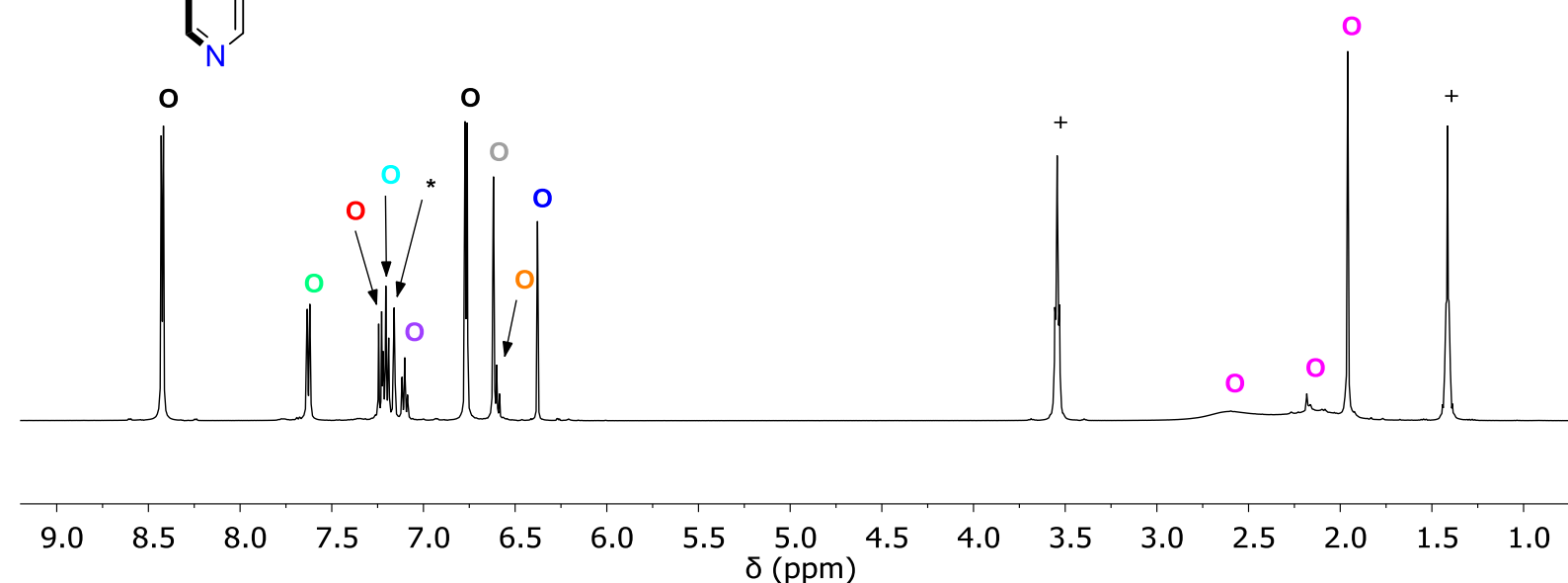

Figure S13: ${ }^{13} \mathrm{C}\left\{{ }^{1} \mathrm{H}\right\} \quad$ NMR spectrum $\left(\mathrm{C}_{6} \mathrm{D}_{6}, \quad 125 \mathrm{MHz}, \quad 298 \mathrm{~K}\right) \quad$ of $\quad\left({ }^{\mathrm{Mes}, \mathrm{Ph}} \mathrm{L}\right) \mathrm{BiI}(4,4 \text { '-bipy })_{2}$ $\left(\mathbf{4} \cdot\left(\mathbf{4}, \mathbf{4}^{\prime}-\text {-bipy }\right)_{2}\right) .+$ denotes thf. $*$ denotes $\mathrm{C}_{6} \mathrm{D}_{6}$.

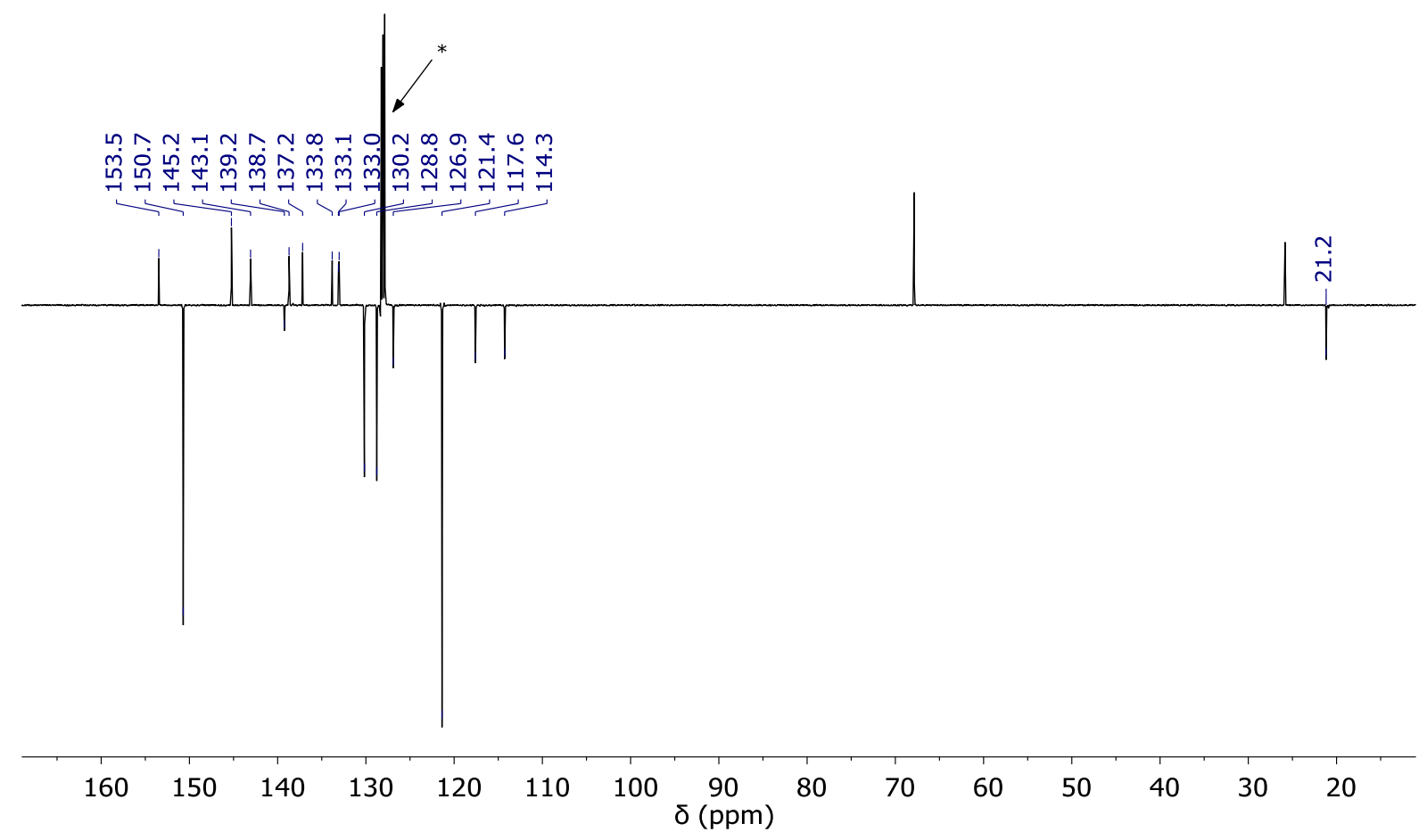

Figure S14: ${ }^{13} \mathrm{C}\left\{{ }^{1} \mathrm{H}\right\}$ APT (attached proton test) NMR spectrum $\left(\mathrm{C}_{6} \mathrm{D}_{6}, 125 \mathrm{MHz}, 298 \mathrm{~K}\right)$ of $\left({ }^{\text {Mes,Ph }} \mathrm{L}\right) \mathrm{BiI}\left(4,4^{\prime}\right.$-bipy) $\left(\mathbf{4} \cdot\left(\mathbf{4}, \mathbf{4}^{\prime} \text {-bipy }\right)_{2}\right) .+$ denotes thf. * denotes $\mathrm{C}_{6} \mathrm{D}_{6}$. 


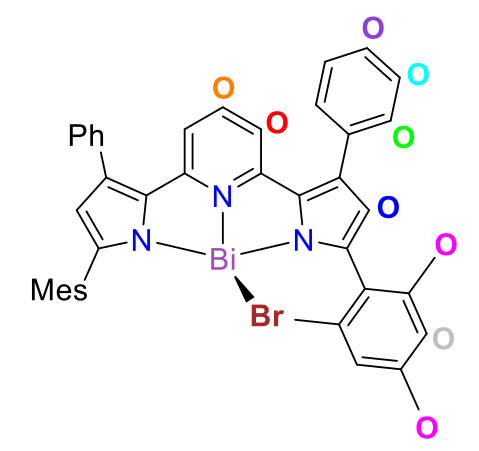
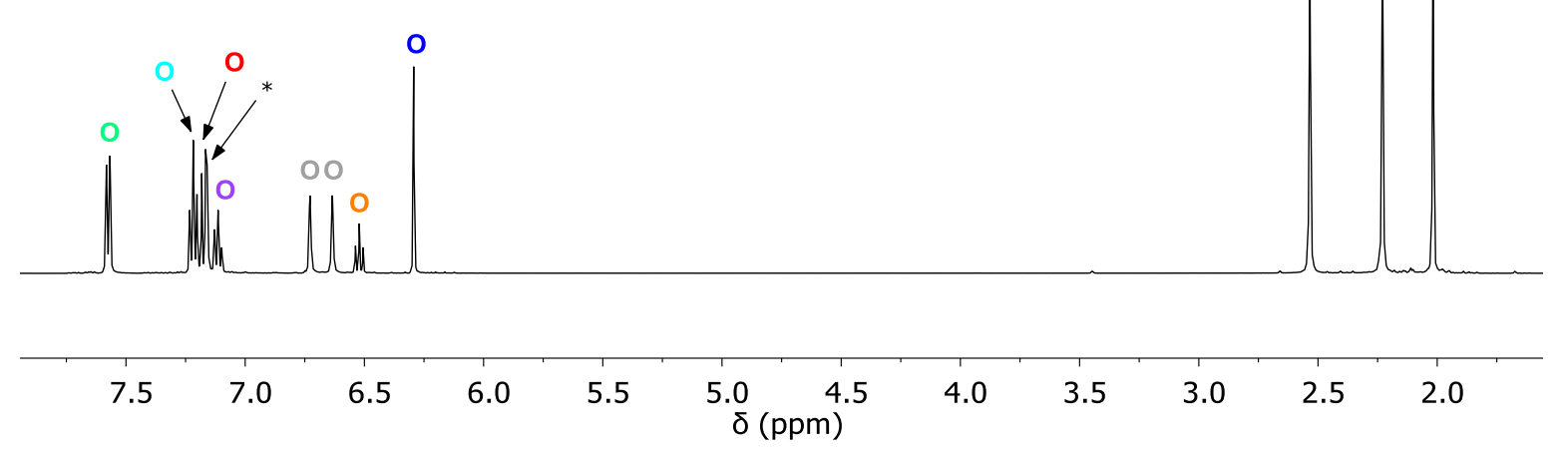

Figure S15: ${ }^{1} \mathrm{H}$ NMR spectrum $\left(\mathrm{C}_{6} \mathrm{D}_{6}, 500 \mathrm{MHz}, 298 \mathrm{~K}\right)$ of $\left({ }^{\mathrm{Mes}, \mathrm{Ph}} \mathrm{L}\right) \mathrm{BiBr}(\mathbf{5})$. * denotes residual protio solvent fraction of $\mathrm{C}_{6} \mathrm{D}_{6}$.

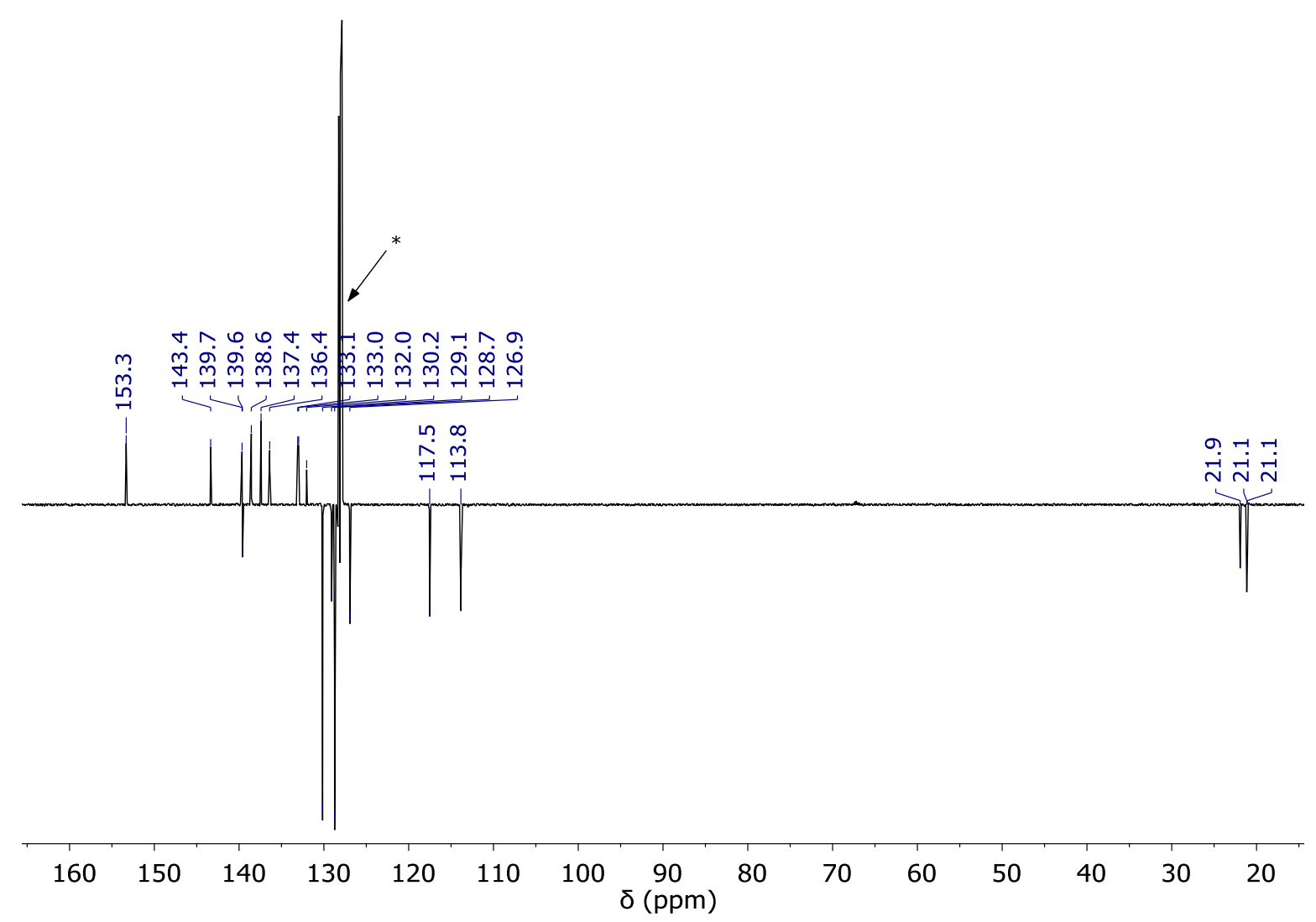

Figure S16: ${ }^{13} \mathrm{C}\left\{{ }^{1} \mathrm{H}\right\}$ APT (attached proton test) NMR spectrum $\left(\mathrm{C}_{6} \mathrm{D}_{6}, 125 \mathrm{MHz}, 298 \mathrm{~K}\right)$ of $\left({ }^{\mathrm{Mes}, \mathrm{Ph}} \mathrm{L}\right) \mathrm{BiBr}(\mathbf{5}) .{ }^{*}$ denotes $\mathrm{C}_{6} \mathrm{D}_{6}$. 


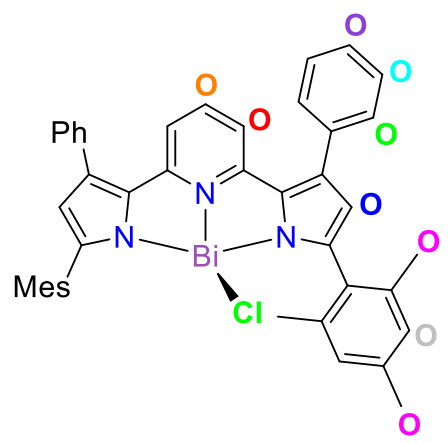

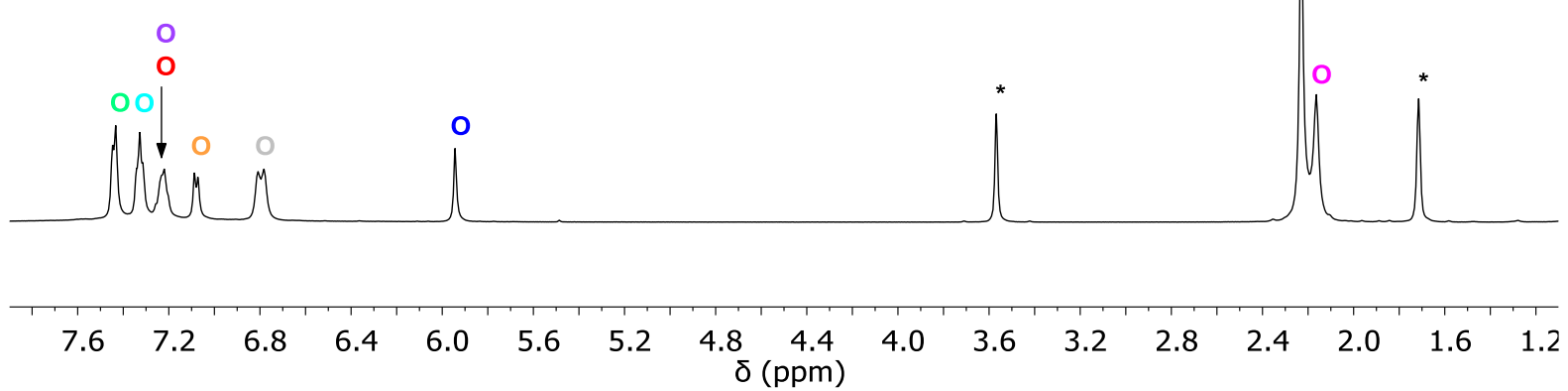

Figure S17: ${ }^{1} \mathrm{H}$ NMR spectrum $\left(\mathrm{C}_{4} \mathrm{D}_{8} \mathrm{O}, 500 \mathrm{MHz}, 298 \mathrm{~K}\right)$ of $\left({ }^{\mathrm{Mes}, \mathrm{Ph}} \mathrm{L}\right) \mathrm{BiCl}(\mathbf{6})$. * denotes residual protio solvent fraction of $\mathrm{C}_{4} \mathrm{D}_{8} \mathrm{O}$.

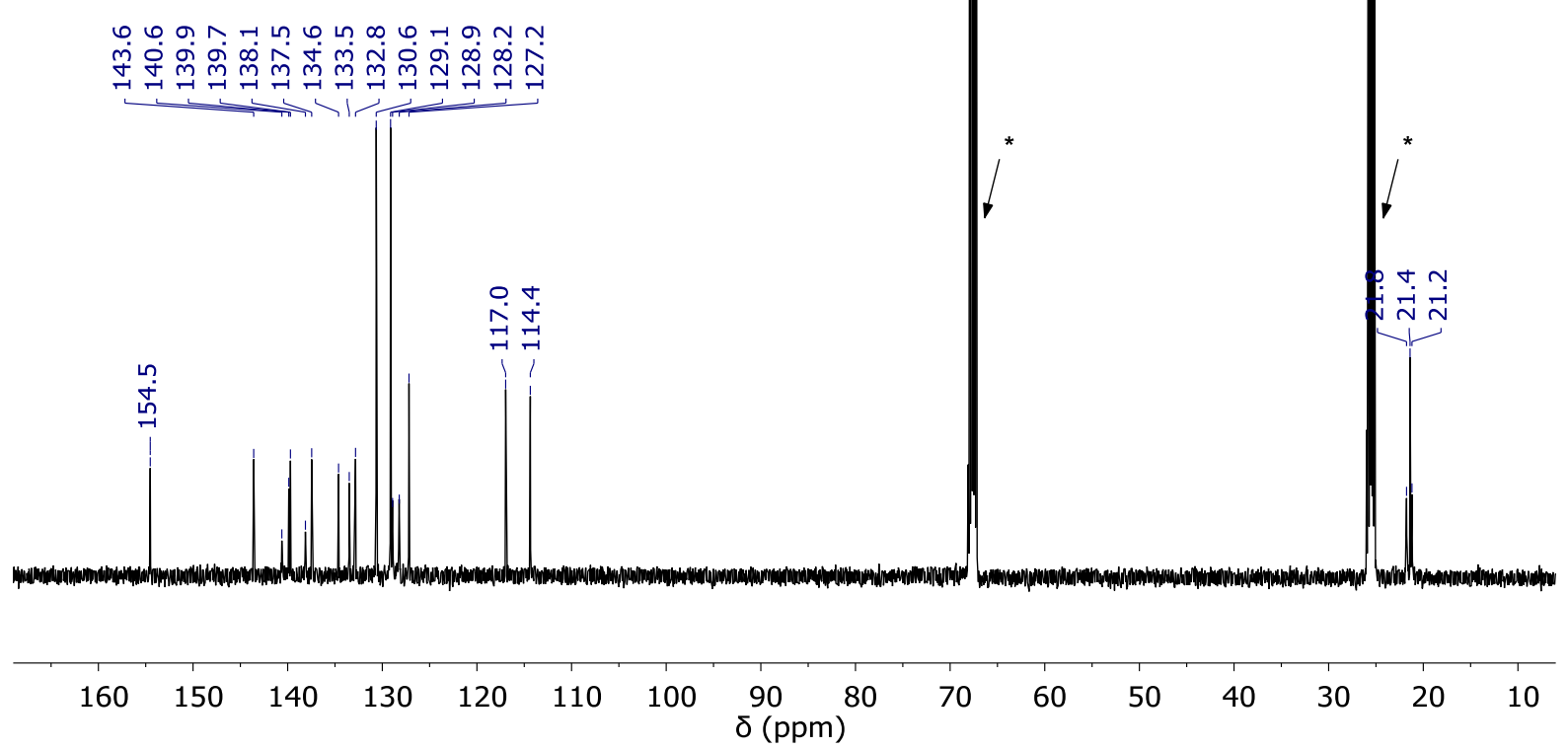

Figure S18: ${ }^{13} \mathrm{C}\left\{{ }^{1} \mathrm{H}\right\} \quad \mathrm{NMR}$ spectrum $\left(\mathrm{C}_{4} \mathrm{D}_{8} \mathrm{O}, \quad 125 \mathrm{MHz}, \quad 298 \mathrm{~K}\right)$ of $\left({ }^{\mathrm{Mes}, \mathrm{Ph}} \mathrm{L}\right) \mathrm{BiCl} \quad(6)$. $*$ denotes $\mathrm{C}_{4} \mathrm{D}_{8} \mathrm{O}$. 


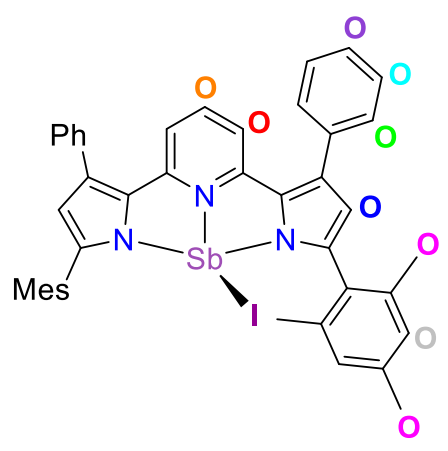
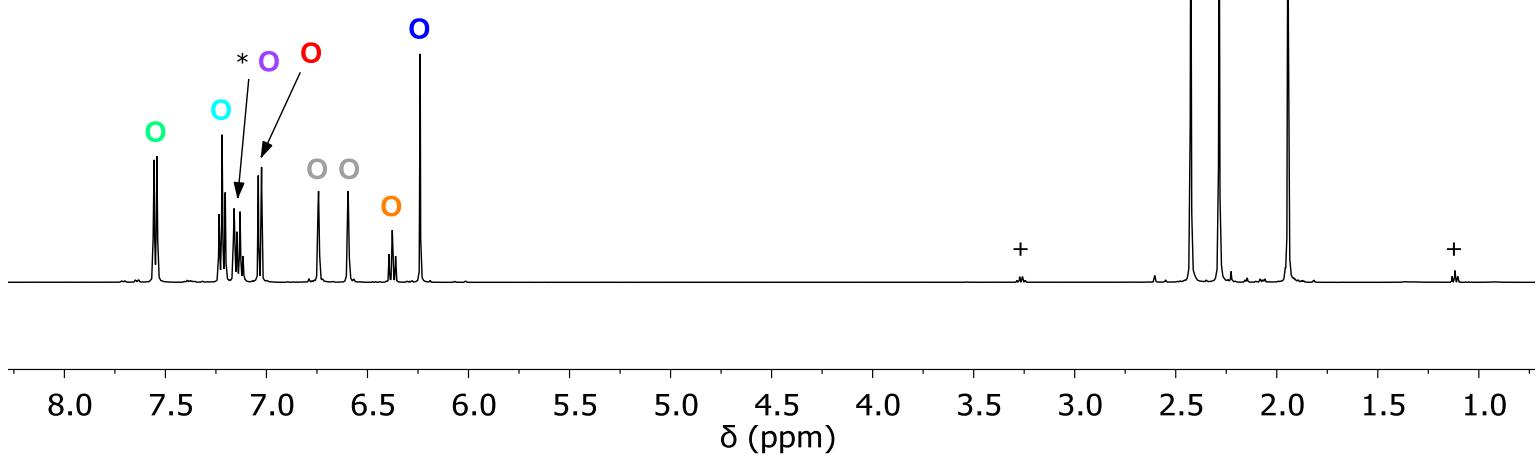

Figure S19: ${ }^{1} \mathrm{H}$ NMR spectrum $\left(\mathrm{C}_{6} \mathrm{D}_{6}, 500 \mathrm{MHz}, 298 \mathrm{~K}\right)$ of $\left({ }^{\mathrm{Mes}, \mathrm{Ph}} \mathrm{L}\right) \mathrm{SbI}(7) .+$ denotes residual $\mathrm{Et}_{2} \mathrm{O} .{ }^{*}$ denotes residual protio solvent fraction of $\mathrm{C}_{6} \mathrm{D}_{6}$.

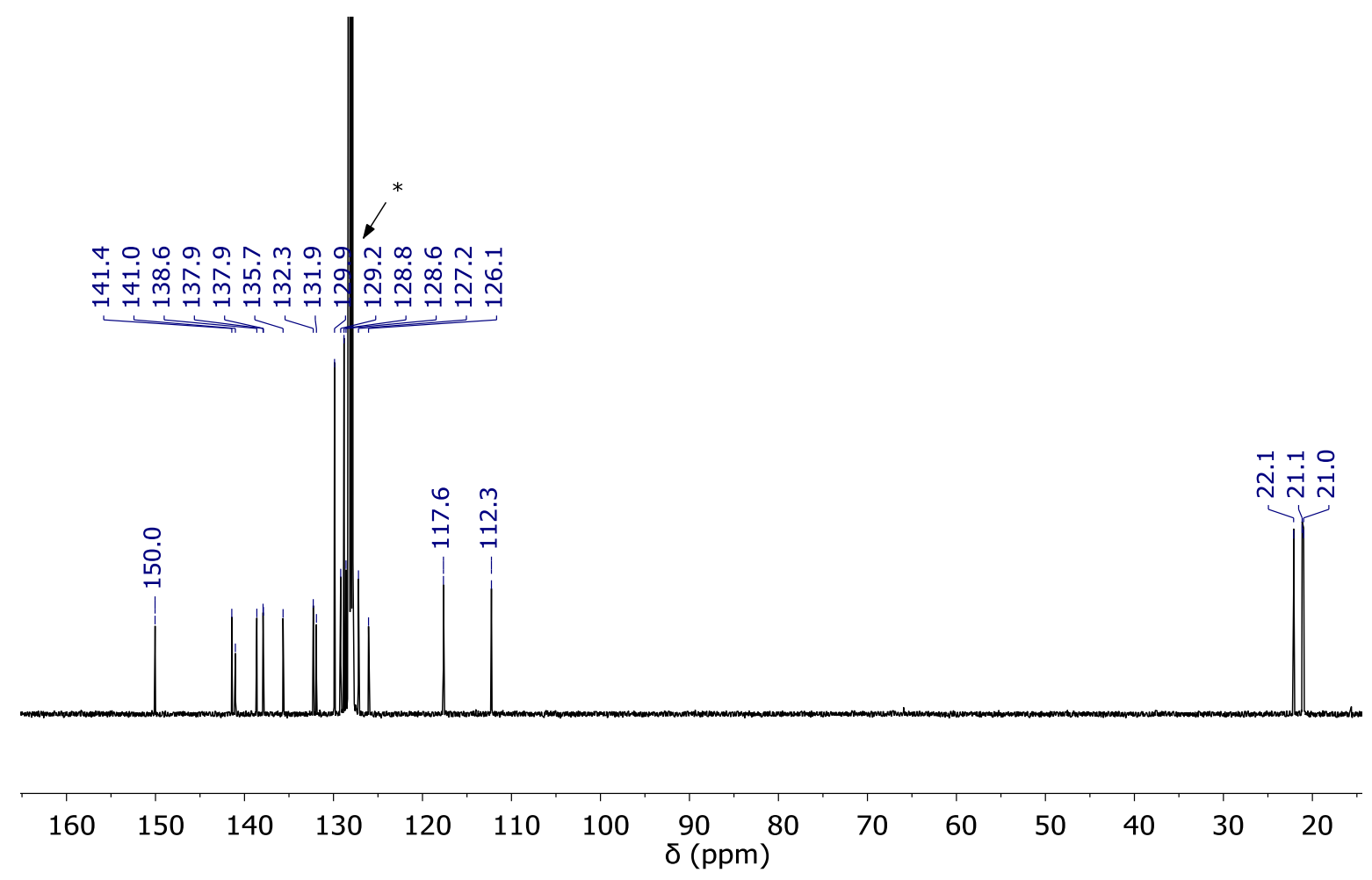

Figure S20: ${ }^{13} \mathrm{C}\left\{{ }^{1} \mathrm{H}\right\}$ NMR spectrum $\left(\mathrm{C}_{6} \mathrm{D}_{6}, 125 \mathrm{MHz}, 298 \mathrm{~K}\right)$ of $\left({ }^{\mathrm{Mes}, \mathrm{Ph}} \mathrm{L}\right) \mathrm{SbI}(\mathbf{7}) . *$ denotes $\mathrm{C}_{6} \mathrm{D}_{6}$. 

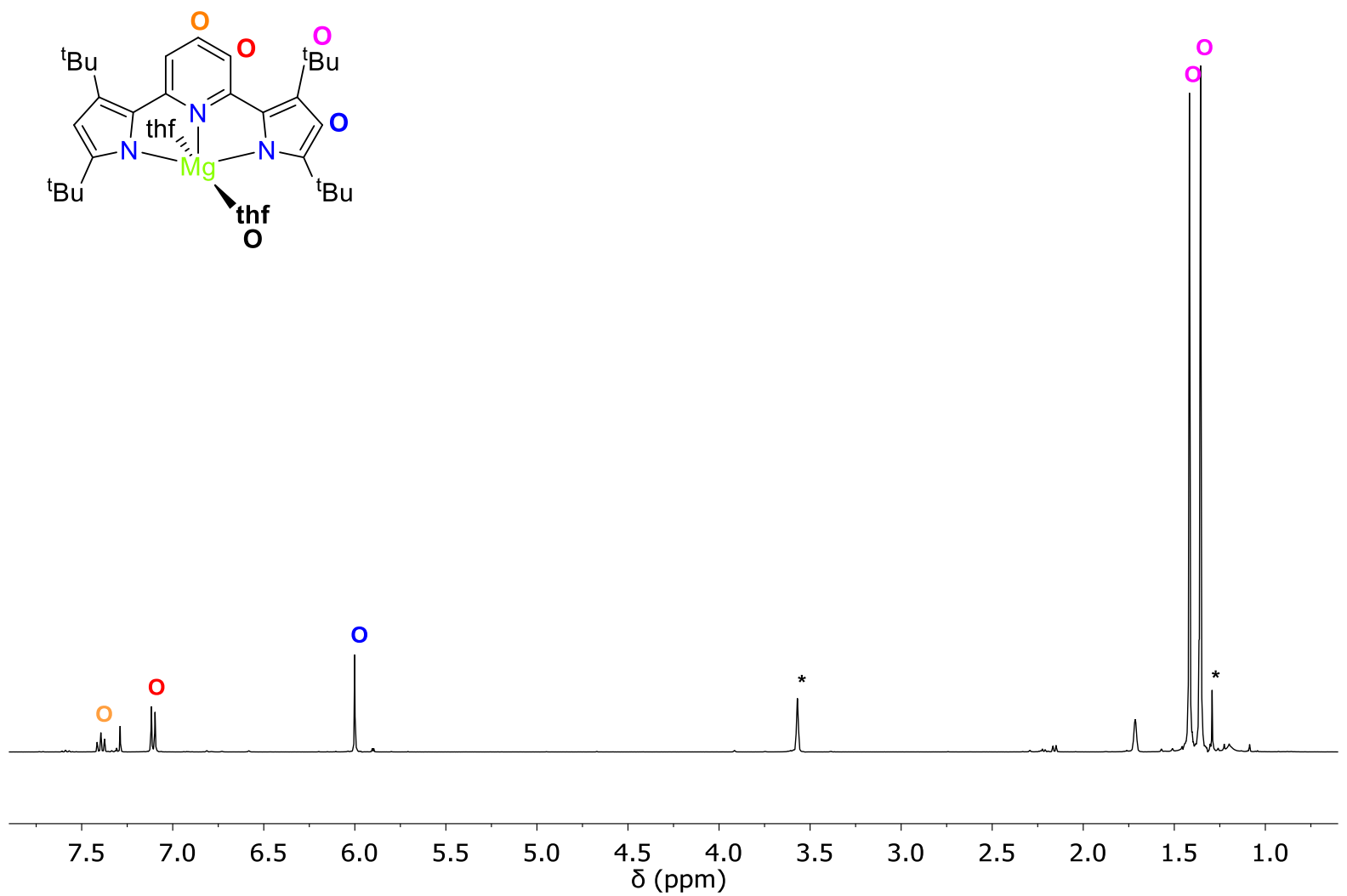

Figure S21: ${ }^{1} \mathrm{H}$ NMR spectrum $\left(\mathrm{C}_{4} \mathrm{D}_{8} \mathrm{O}, 500 \mathrm{MHz}, 298 \mathrm{~K}\right)$ of $\left({ }^{\mathrm{t} B u},{ }^{\mathrm{B} u} \mathrm{~L}\right) \mathrm{Mg}(\operatorname{thf})_{2}(\mathbf{8}) . *$ denotes residual protio solvent fraction of $\mathrm{C}_{4} \mathrm{D}_{8} \mathrm{O}$.

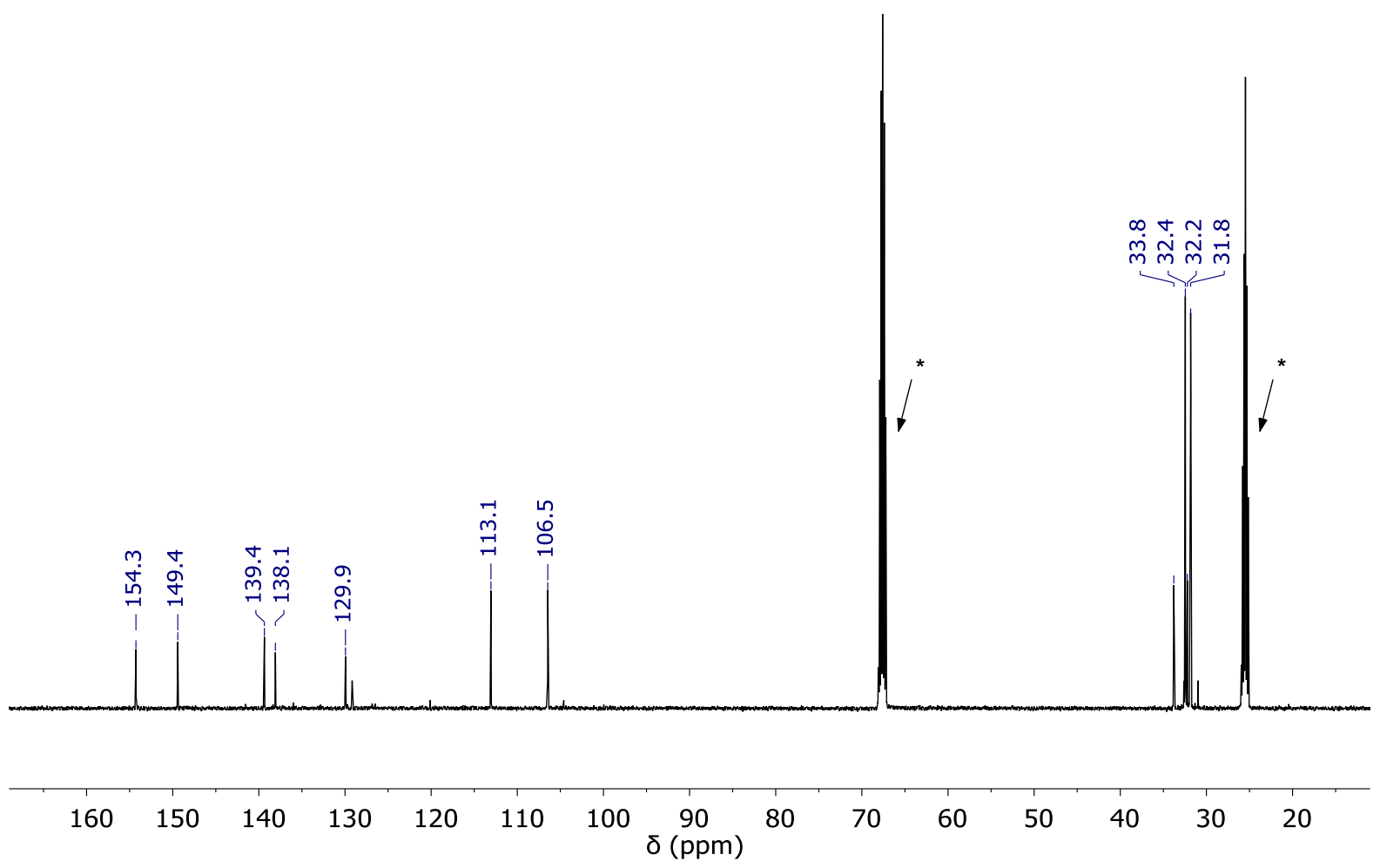

Figure S22: ${ }^{13} \mathrm{C}\left\{{ }^{1} \mathrm{H}\right\} \quad \mathrm{NMR}$ spectrum $\left(\mathrm{C}_{4} \mathrm{D}_{8} \mathrm{O}, \quad 125 \mathrm{MHz}, 298 \mathrm{~K}\right)$ of $\left({ }^{\mathrm{t} B u},{ }^{\mathrm{t}} \mathrm{Bu} \mathrm{L}\right) \mathrm{Mg}(\operatorname{thf})_{2} \quad$ (8). * denotes $\mathrm{C}_{4} \mathrm{D}_{8} \mathrm{O}$. 

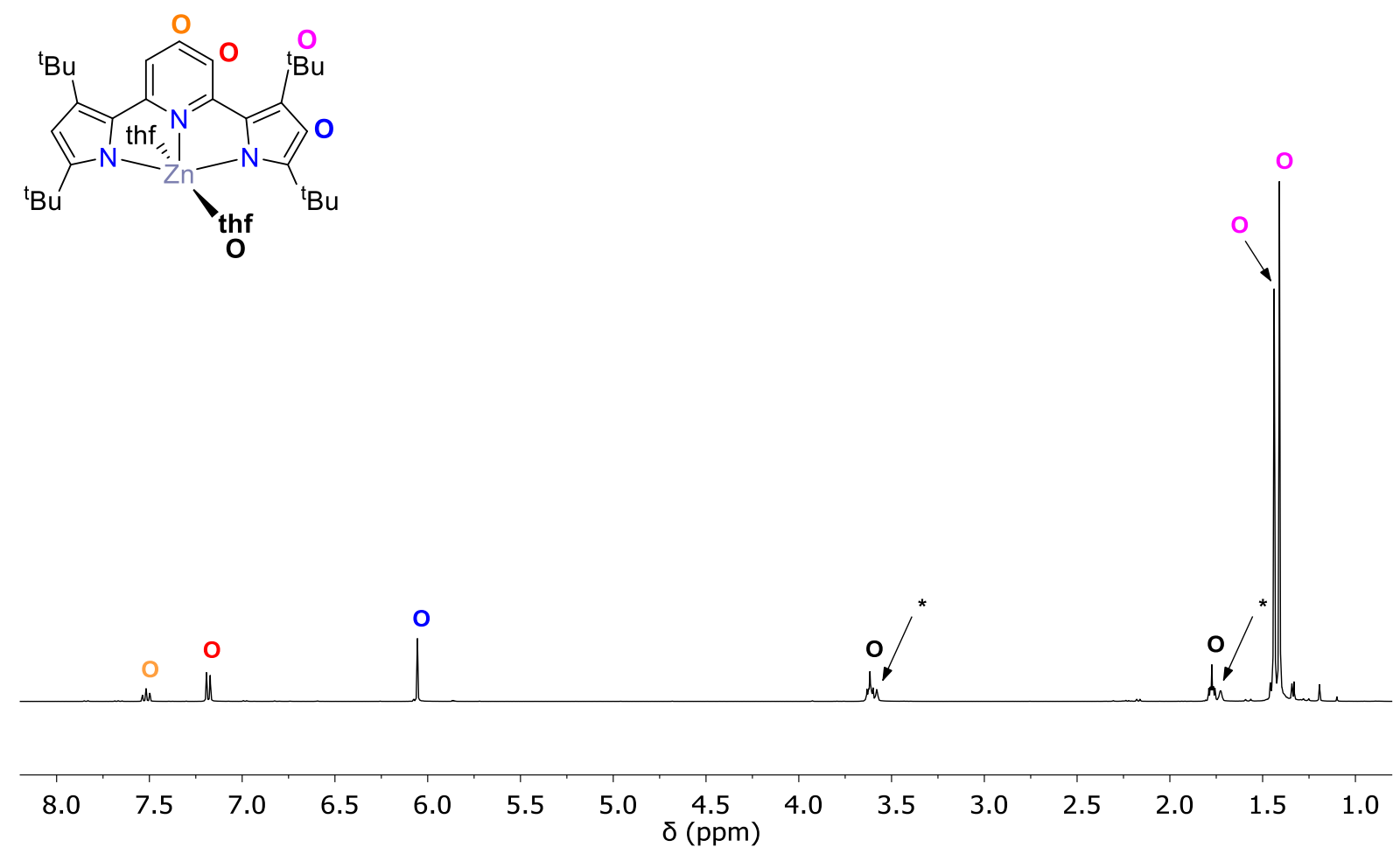

Figure S23: ${ }^{1} \mathrm{H}$ NMR spectrum $\left(\mathrm{C}_{4} \mathrm{D}_{8} \mathrm{O}, 500 \mathrm{MHz}, 298 \mathrm{~K}\right)$ of $\left({ }^{{ }^{\mathrm{B} u},{ }^{\mathrm{t}} \mathrm{Bu}} \mathrm{L}\right) \mathrm{Zn}(\operatorname{thf})_{2}(\mathbf{9}) . *$ denotes residual protio solvent fraction of $\mathrm{C}_{4} \mathrm{D}_{8} \mathrm{O}$.

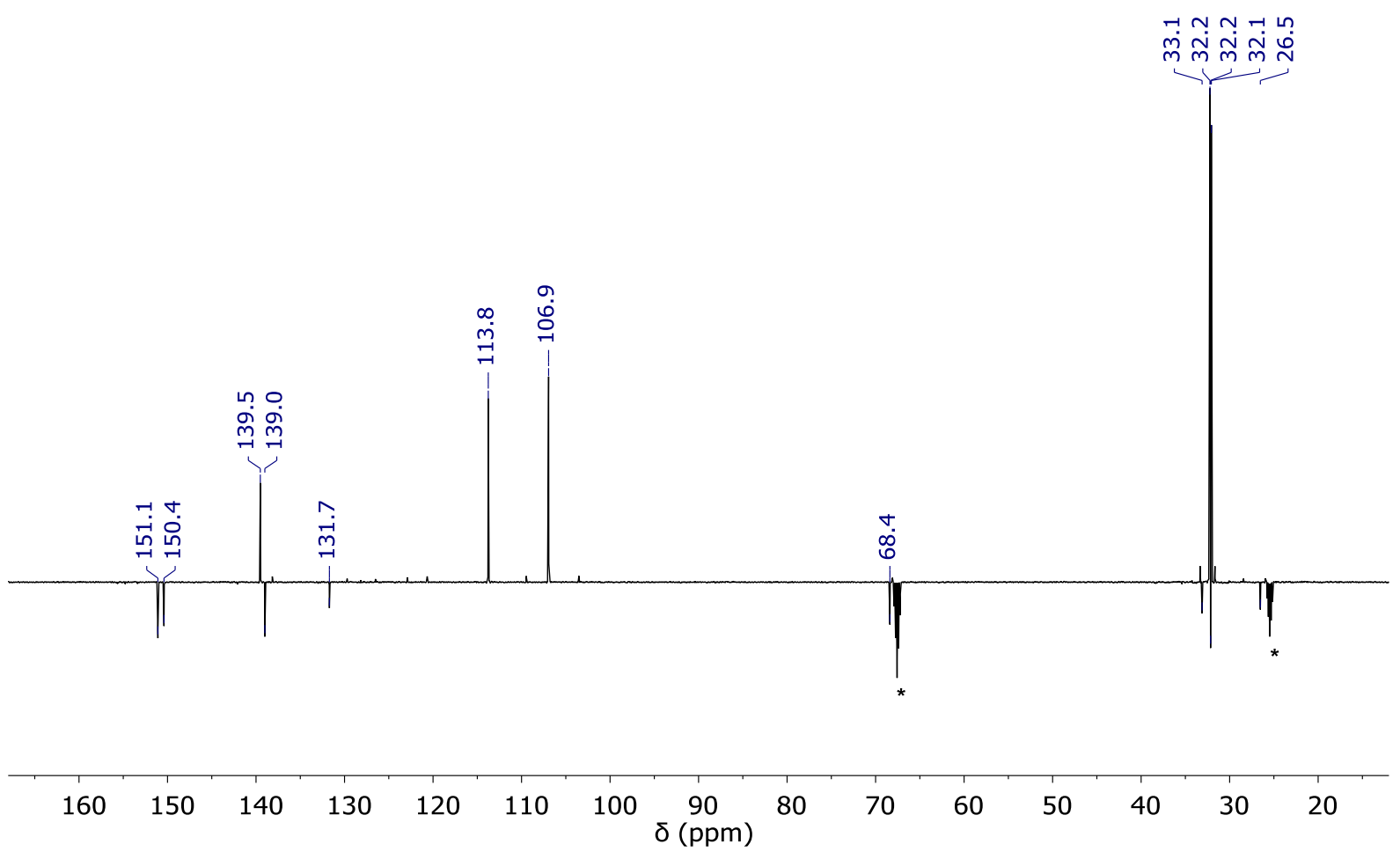

Figure S24: ${ }^{13} \mathrm{C}\left\{{ }^{1} \mathrm{H}\right\}$ APT (attached proton test) NMR spectrum $\left(\mathrm{C}_{4} \mathrm{D}_{8} \mathrm{O}, 125 \mathrm{MHz}, 298 \mathrm{~K}\right)$ of $\left({ }^{\mathrm{B} B u},{ }^{\mathrm{B} u} \mathrm{~L}\right) \mathrm{Zn}(\text { thf })_{2}(\mathbf{9}) . *$ denotes $\mathrm{C}_{4} \mathrm{D}_{8} \mathrm{O}$. 


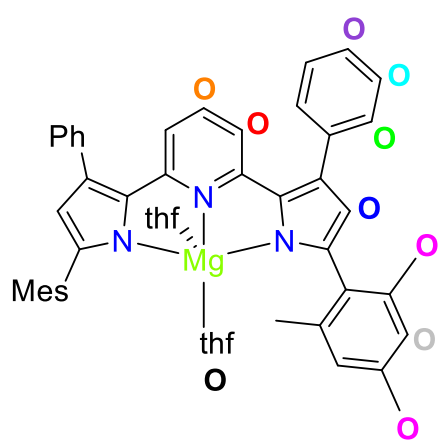
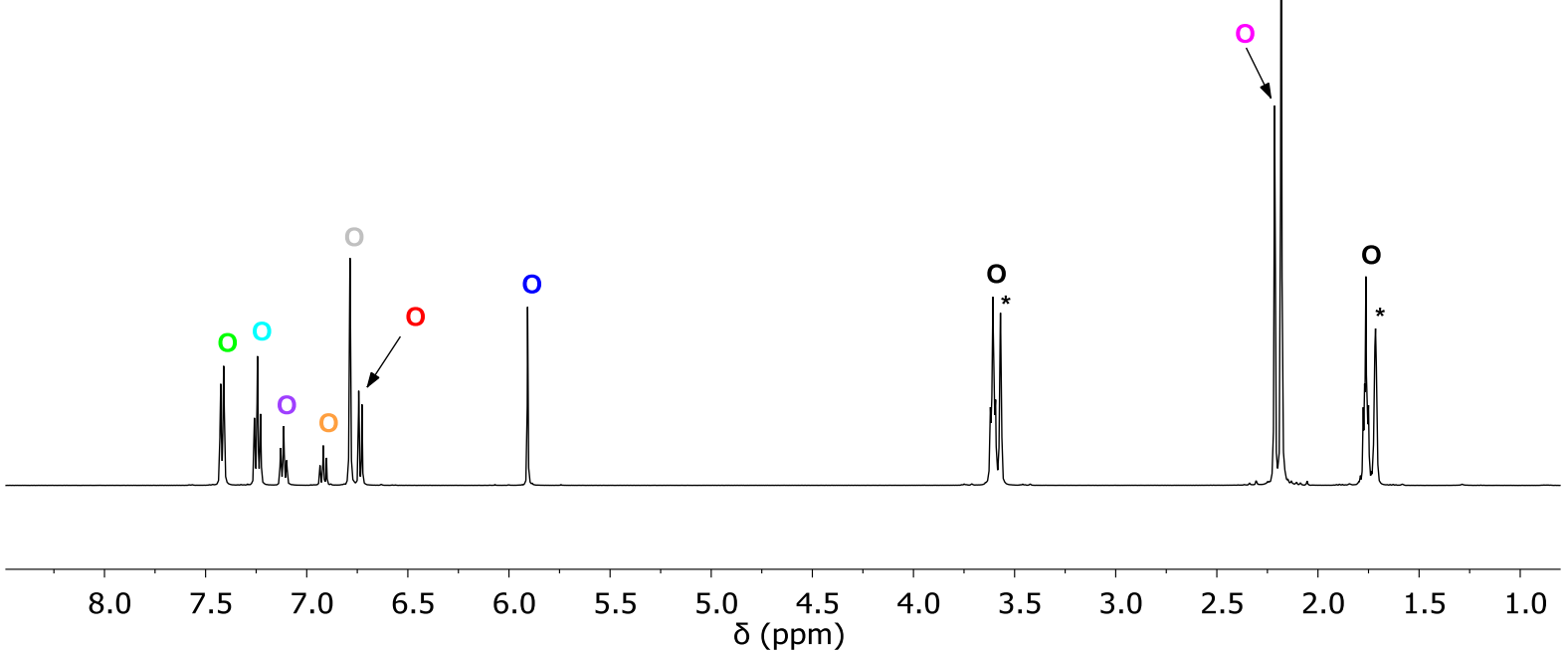

Figure S25: ${ }^{1} \mathrm{H}$ NMR spectrum $\left(\mathrm{C}_{4} \mathrm{D}_{8} \mathrm{O}, 500 \mathrm{MHz}, 298 \mathrm{~K}\right)$ of $\left({ }^{\mathrm{Mes}, \mathrm{Ph}} \mathrm{L}\right) \mathrm{Mg}(\text { thf })_{2}(\mathbf{1 0}) . *$ denotes residual protio solvent fraction of $\mathrm{C}_{4} \mathrm{D}_{8} \mathrm{O}$.

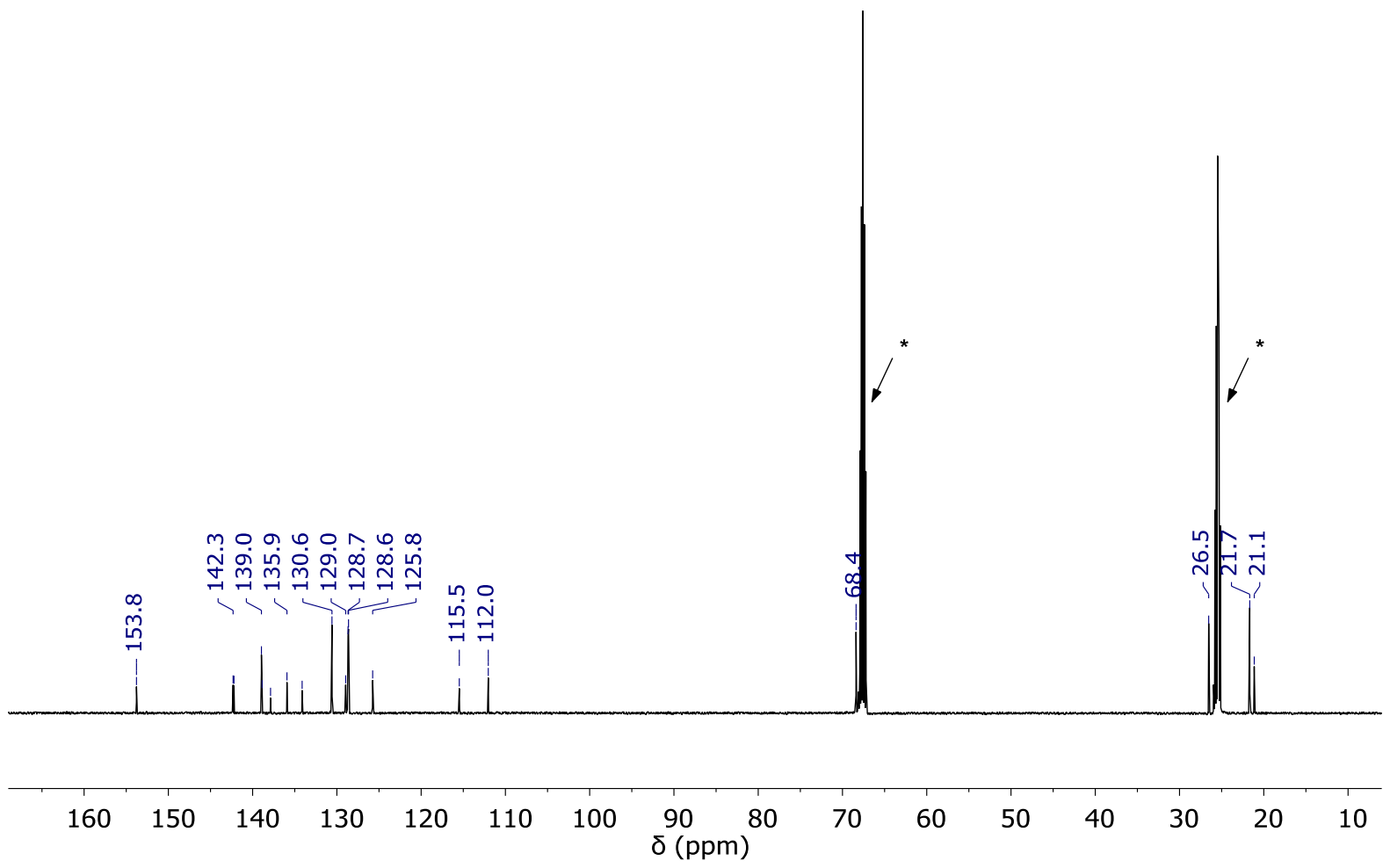

Figure S26: ${ }^{13} \mathrm{C}\left\{{ }^{1} \mathrm{H}\right\} \quad \mathrm{NMR}$ spectrum $\left(\mathrm{C}_{4} \mathrm{D}_{8} \mathrm{O}, 125 \mathrm{MHz}, 298 \mathrm{~K}\right)$ of $\left({ }^{\mathrm{Mes}, \mathrm{Ph}} \mathrm{L}\right) \mathrm{Mg}(\text { thf })_{2}$ (10). * denotes $\mathrm{C}_{4} \mathrm{D}_{8} \mathrm{O}$. 


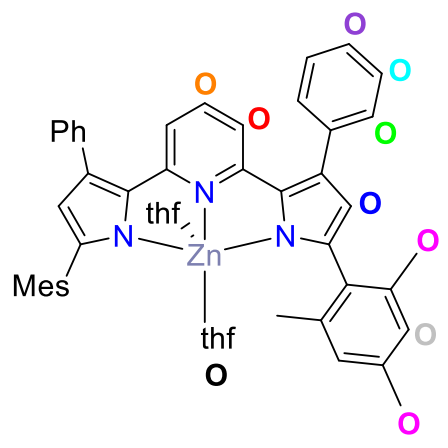

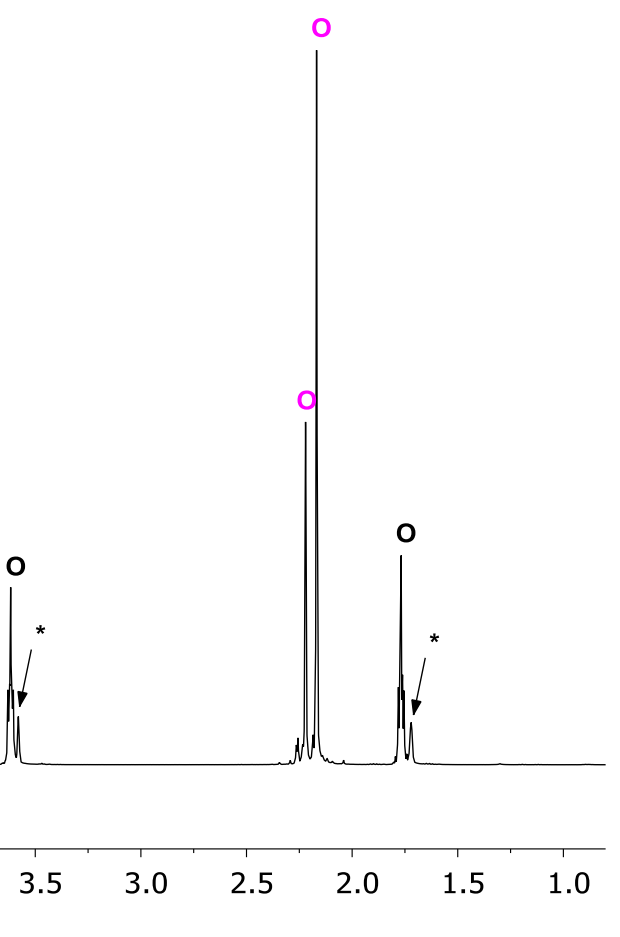

Figure S27: ${ }^{1} \mathrm{H}$ NMR spectrum $\left(\mathrm{C}_{4} \mathrm{D}_{8} \mathrm{O}, 500 \mathrm{MHz}, 298 \mathrm{~K}\right)$ of $\left({ }^{\mathrm{Mes}, \mathrm{Ph}} \mathrm{L}\right) \mathrm{Zn}(\text { thf })_{2}(\mathbf{1 1}) . *$ denotes residual protio solvent fraction of $\mathrm{C}_{4} \mathrm{D}_{8} \mathrm{O}$.

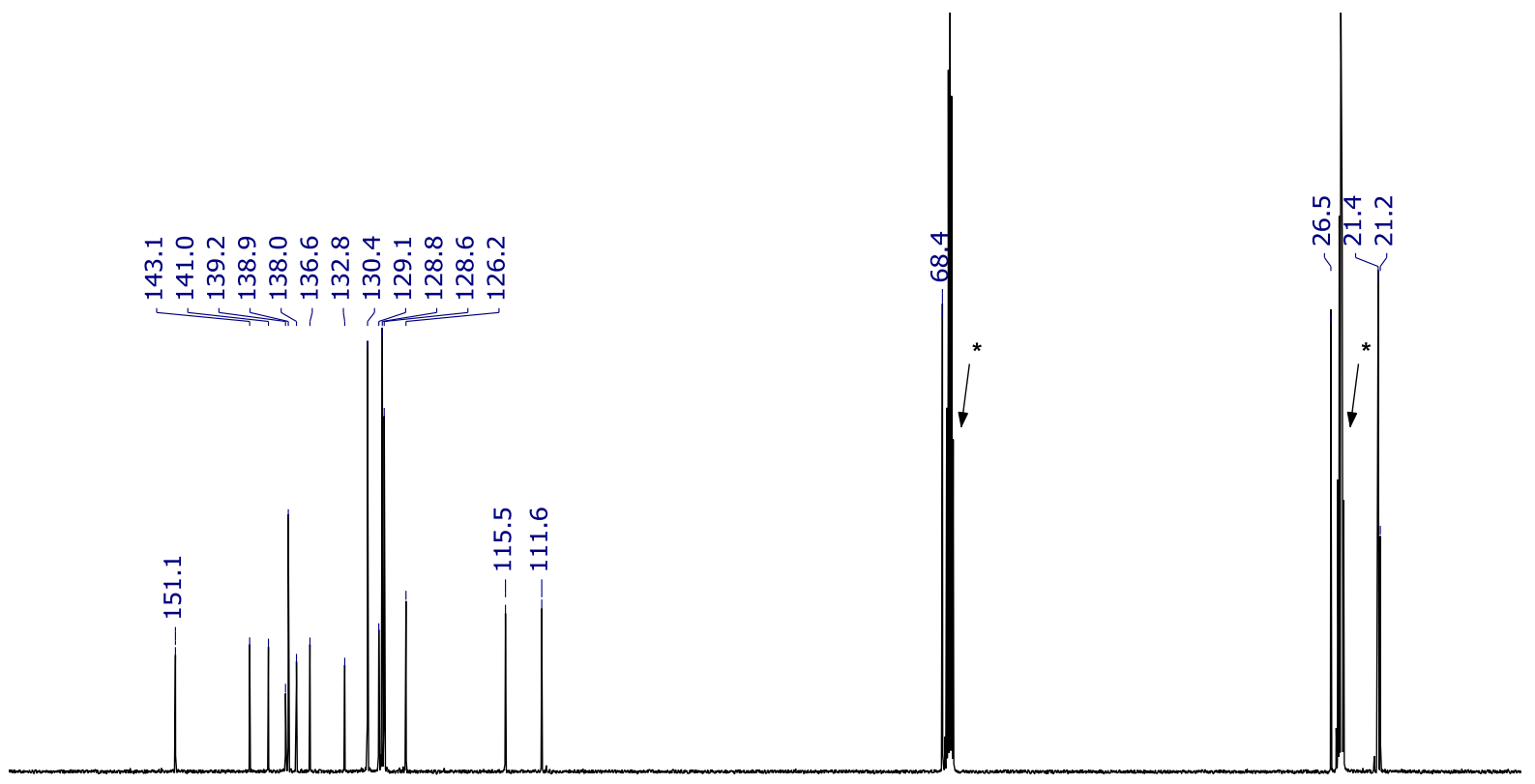

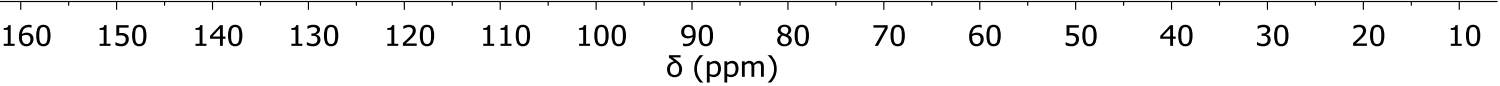

Figure S28: ${ }^{13} \mathrm{C}\left\{{ }^{1} \mathrm{H}\right\} \quad \mathrm{NMR}$ spectrum $\left(\mathrm{C}_{4} \mathrm{D}_{8} \mathrm{O}, \quad 125 \mathrm{MHz}, 298 \mathrm{~K}\right)$ of ( $\left.{ }^{\mathrm{Mes}, \mathrm{Ph}} \mathrm{L}\right) \mathrm{Zn}(\mathrm{thf})_{2} \quad$ (11). * denotes $\mathrm{C}_{4} \mathrm{D}_{8} \mathrm{O}$. 

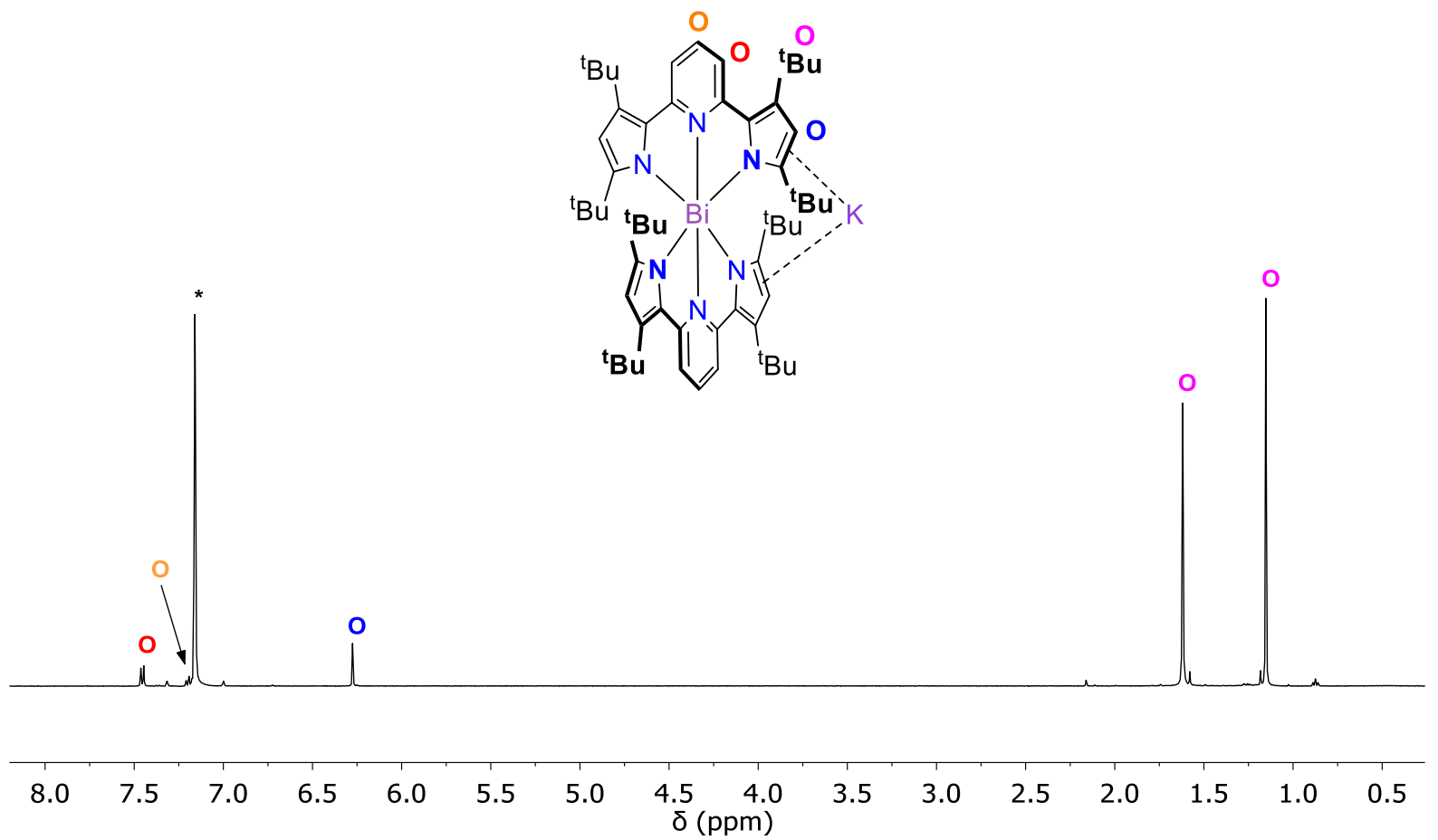

Figure S29: ${ }^{1} \mathrm{H}$ NMR spectrum $\left(\mathrm{C}_{6} \mathrm{D}_{6}, 500 \mathrm{MHz}, 298 \mathrm{~K}\right)$ of single crystals of $\mathrm{K}($ thf $)\left[\left({ }^{\mathrm{t} B \mathrm{Bu}},{ }^{\mathrm{B}} \mathrm{Bu} \mathrm{L}\right)_{2} \mathrm{Bi}\right](\mathbf{1 2})$ following drying under dynamic vacuum. $*$ denotes residual protio solvent fraction of $\mathrm{C}_{6} \mathrm{D}_{6}$.

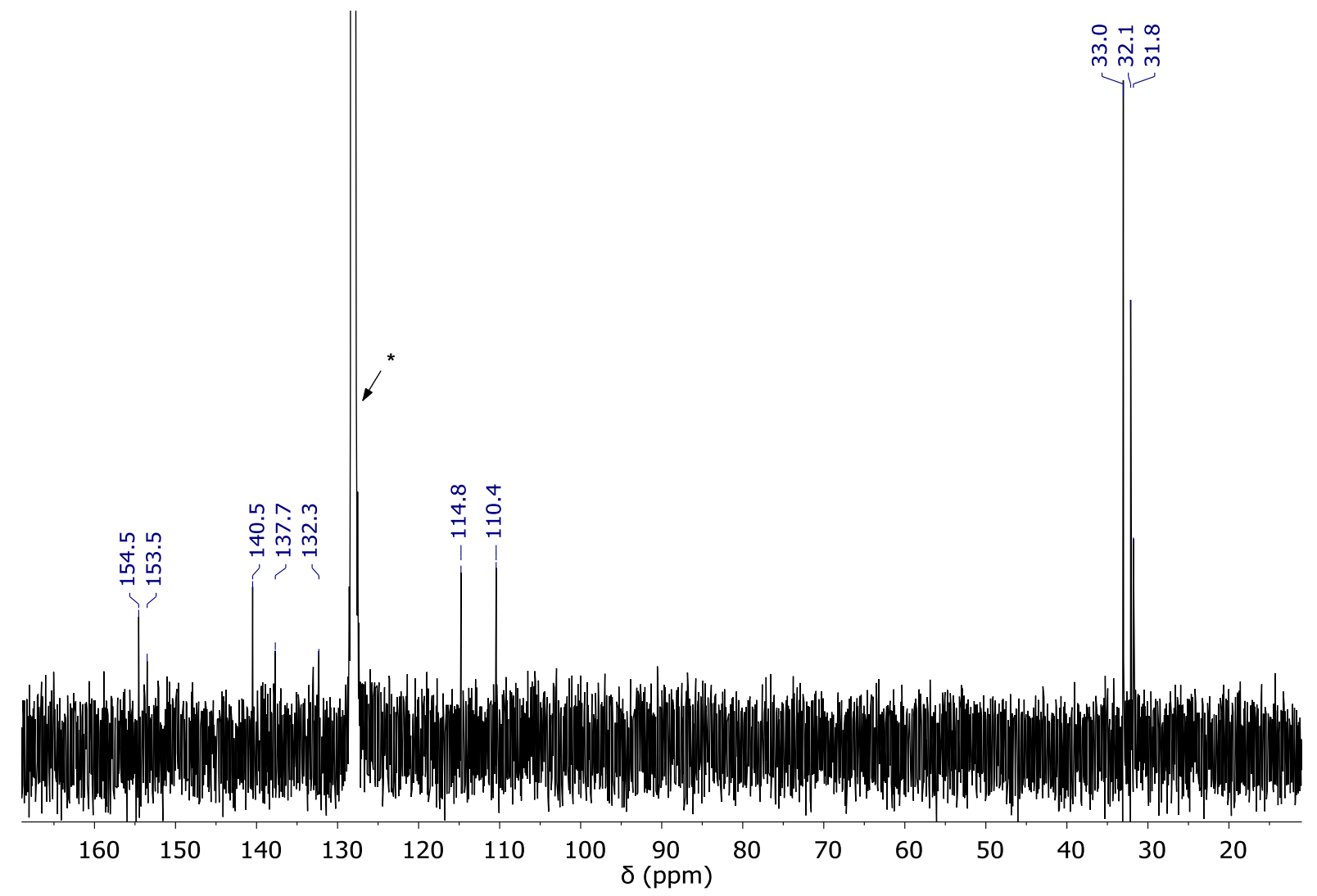

Figure S30: ${ }^{13} \mathrm{C}\left\{{ }^{1} \mathrm{H}\right\}$ NMR spectrum $\left(\mathrm{C}_{6} \mathrm{D}_{6}, 125 \mathrm{MHz}, 298 \mathrm{~K}\right)$ of single crystals of $\mathrm{K}($ thf $)\left[\left({ }^{\mathrm{tBu}},{ }^{\mathrm{t}} \mathrm{Bu} \mathrm{L}\right){ }_{2} \mathrm{Bi}\right]$ (12) following drying under dynamic vacuum. $*$ denotes $\mathrm{C}_{6} \mathrm{D}_{6}$. 

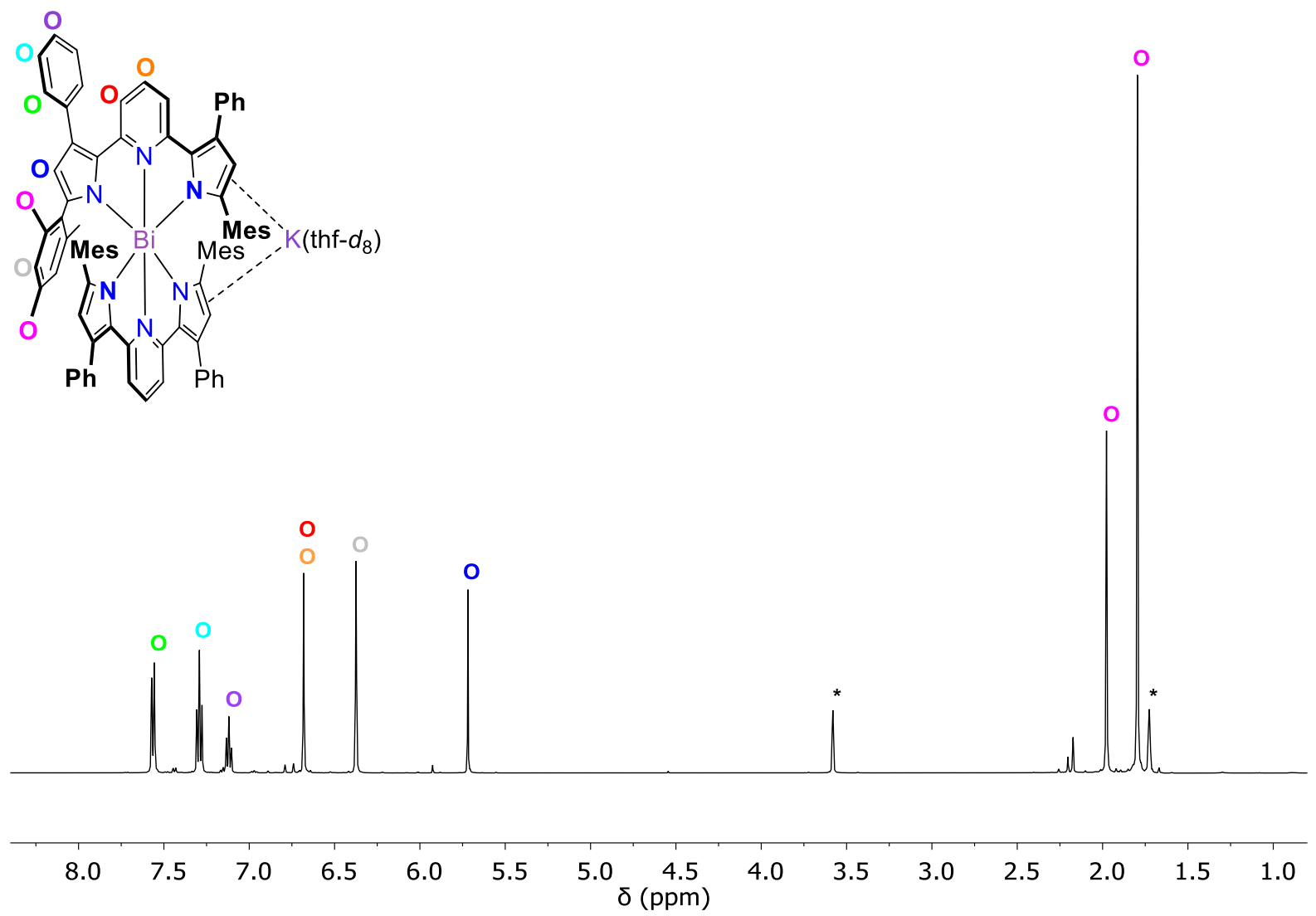

Figure S31: ${ }^{1} \mathrm{H}$ NMR spectrum $\left(\mathrm{C}_{4} \mathrm{D}_{8} \mathrm{O}, 500 \mathrm{MHz}, 298 \mathrm{~K}\right)$ of $\mathrm{K}\left(\right.$ thf $\left.-d_{8}\right)\left[\left({ }^{\mathrm{Mes}, \mathrm{Ph}} \mathrm{L}\right)_{2} \mathrm{Bi}\right](\mathbf{1 3}) . *$ denotes residual protio solvent fraction of $\mathrm{C}_{4} \mathrm{D}_{8} \mathrm{O}$.

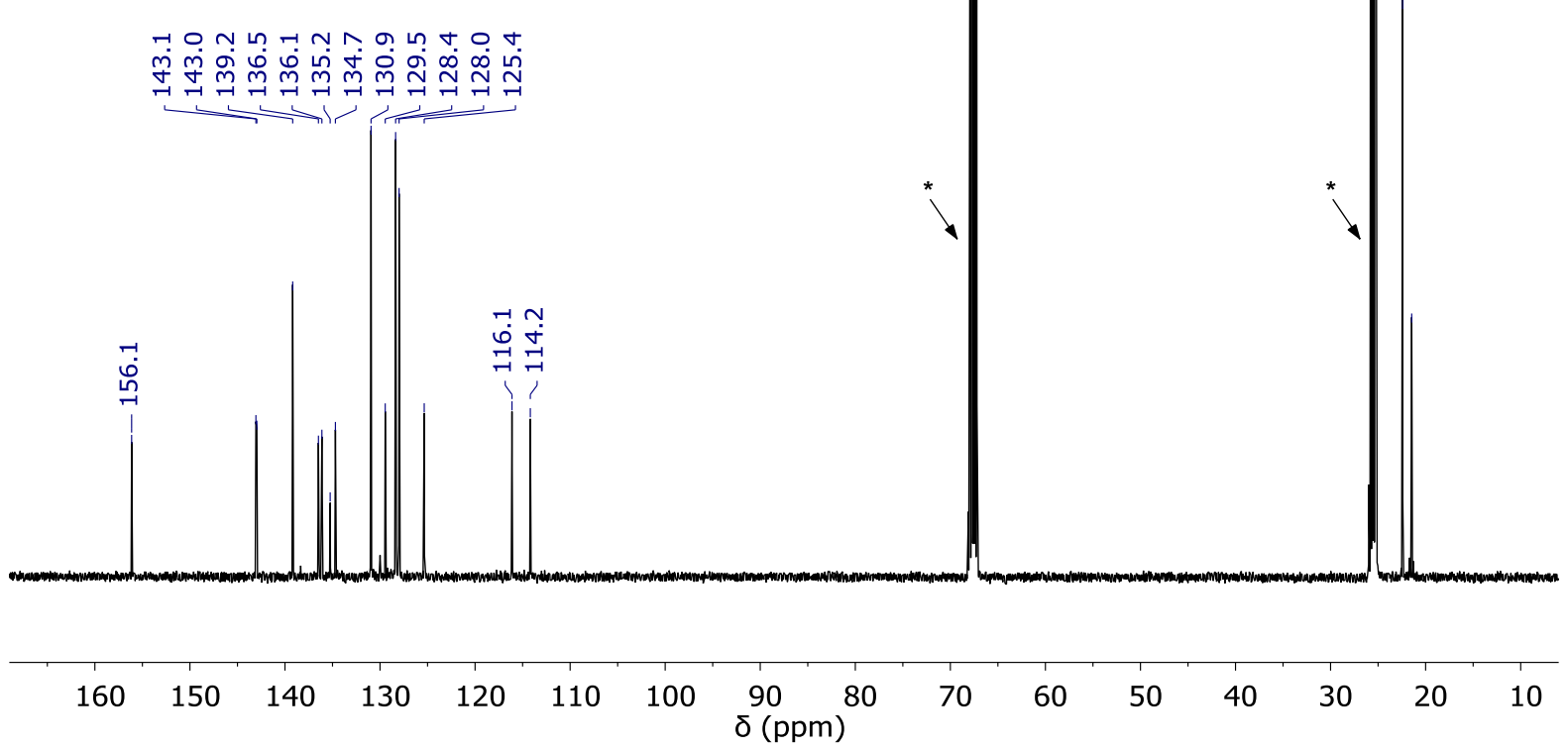

Figure S32: ${ }^{13} \mathrm{C}\left\{{ }^{1} \mathrm{H}\right\}$ NMR spectrum $\left(\mathrm{C}_{4} \mathrm{D}_{8} \mathrm{O}, 125 \mathrm{MHz}, 298 \mathrm{~K}\right)$ of $\mathrm{K}\left(\right.$ thf $\left.-d_{8}\right)\left[\left({ }^{\mathrm{Mes}, \mathrm{Ph}} \mathrm{L}\right)_{2} \mathrm{Bi}\right](\mathbf{1 3})$. * denotes $\mathrm{C}_{4} \mathrm{D}_{8} \mathrm{O}$. 

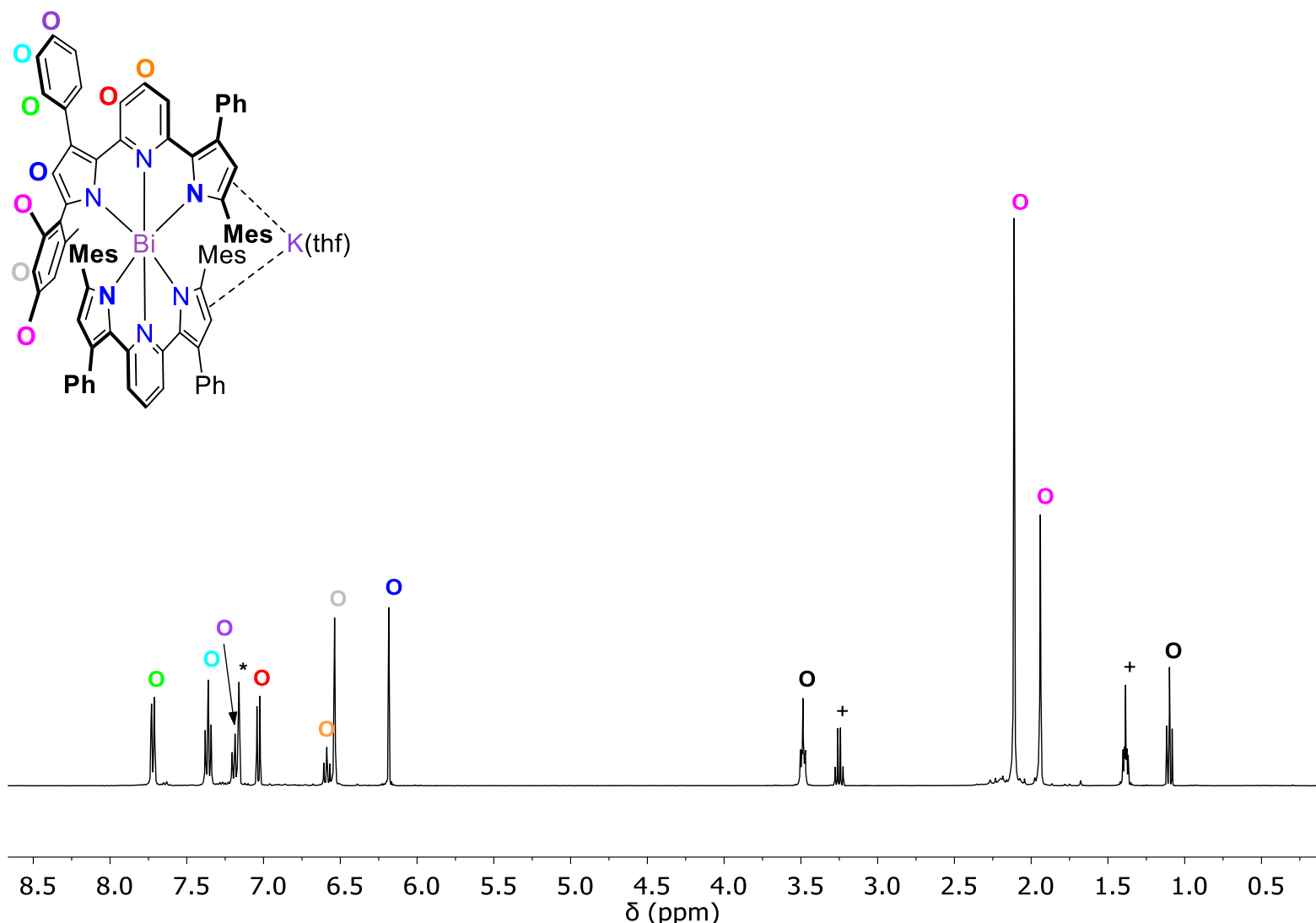

Figure S33: ${ }^{1} \mathrm{H}$ NMR spectrum $\left(\mathrm{C}_{6} \mathrm{D}_{6}, 400 \mathrm{MHz}, 298 \mathrm{~K}\right)$ of $\mathrm{K}(\mathrm{thf})\left[\left({ }^{\mathrm{Mes}, \mathrm{Ph}} \mathrm{L}\right){ }_{2} \mathrm{Bi}\right](\mathbf{1 3})$. + denotes $\mathrm{Et}_{2} \mathrm{O}$. $*$ denotes residual protio solvent fraction of $\mathrm{C}_{6} \mathrm{D}_{6}$. 

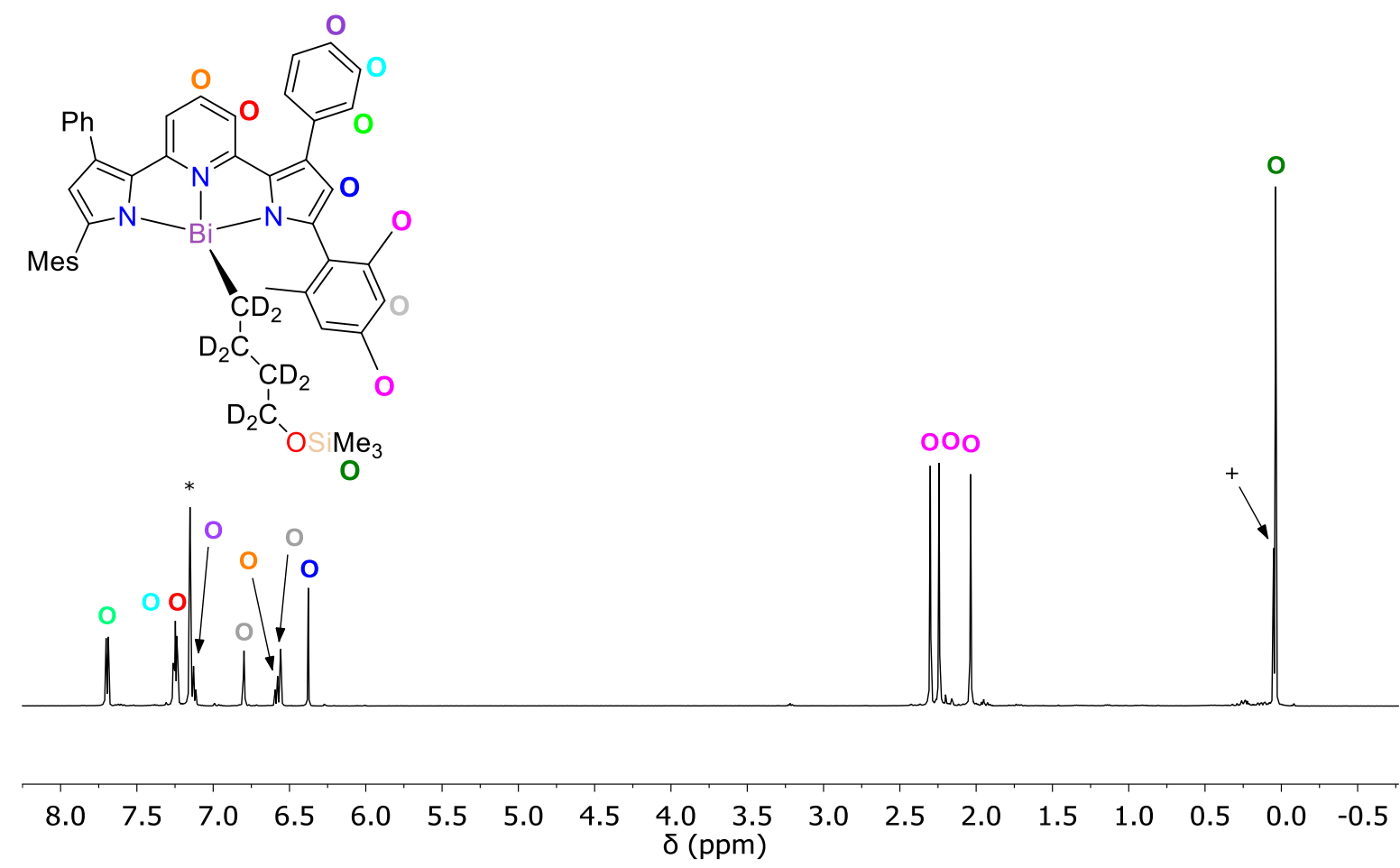

Figure S34: ${ }^{1} \mathrm{H}$ NMR spectrum $\left(\mathrm{C}_{6} \mathrm{D}_{6}, 500 \mathrm{MHz}, 298 \mathrm{~K}\right)$ of $\left({ }^{\mathrm{Mes}, \mathrm{Ph}} \mathrm{L}\right) \mathrm{Bi}\left(\left\{\mathrm{CD}_{2}\right\}_{4} \mathrm{OSiMe}_{3}\right)$ (14). + denotes residual $\mathrm{Me}_{3} \mathrm{SiI}$. * denotes residual protio solvent fraction of $\mathrm{C}_{6} \mathrm{D}_{6}$.

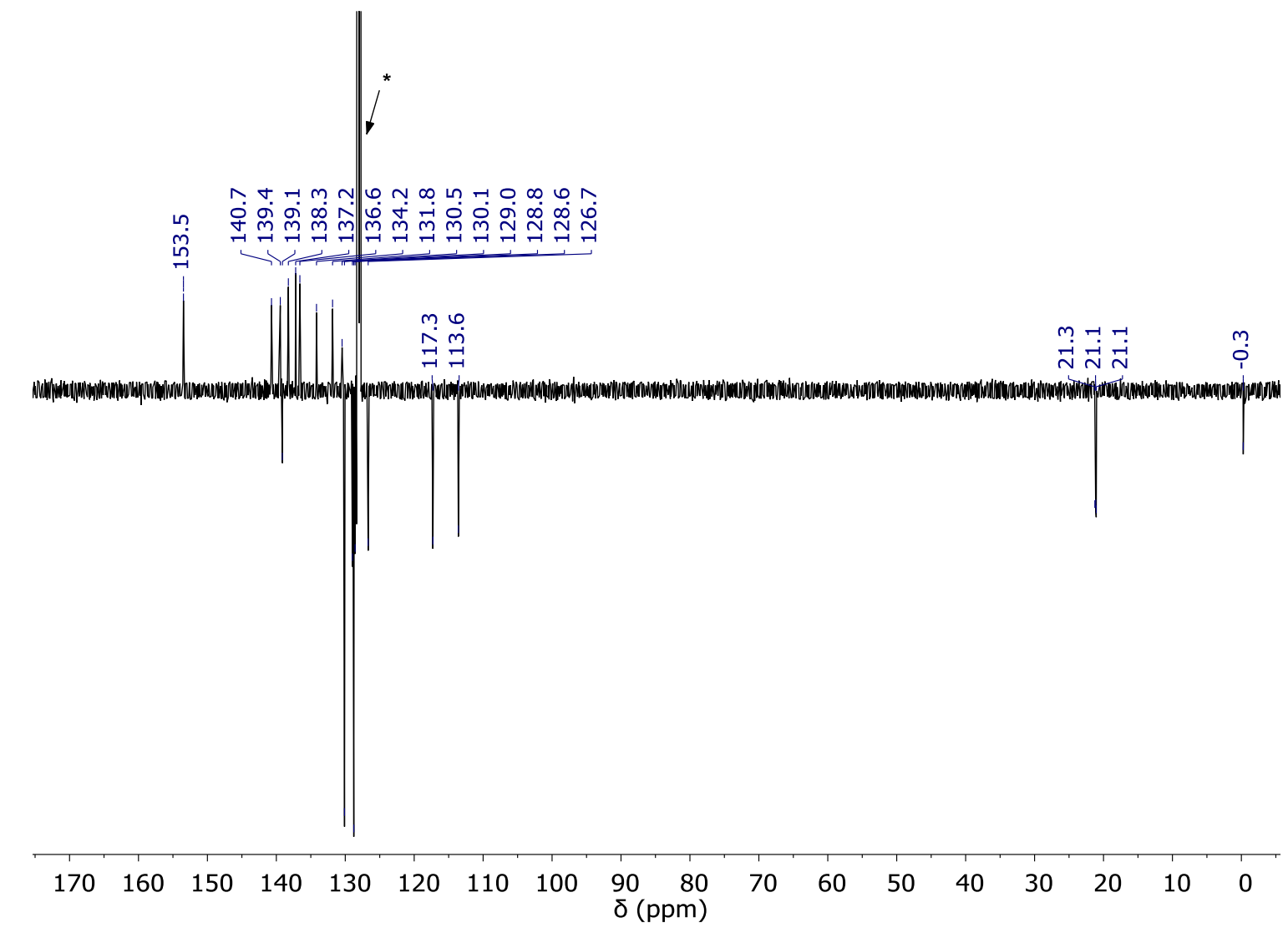

Figure S35: ${ }^{13} \mathrm{C}\left\{{ }^{1} \mathrm{H}\right\}$ APT (attached proton test) NMR spectrum $\left(\mathrm{C}_{6} \mathrm{D}_{6}, 125 \mathrm{MHz}, 298 \mathrm{~K}\right)$ of $\left({ }^{\mathrm{Mes}, \mathrm{Ph}} \mathrm{L}\right) \mathrm{Bi}\left(\left\{\mathrm{CD}_{2}\right\}_{4} \mathrm{OSiMe}_{3}\right)(\mathbf{1 4}) . *$ denotes $\mathrm{C}_{6} \mathrm{D}_{6}$. 

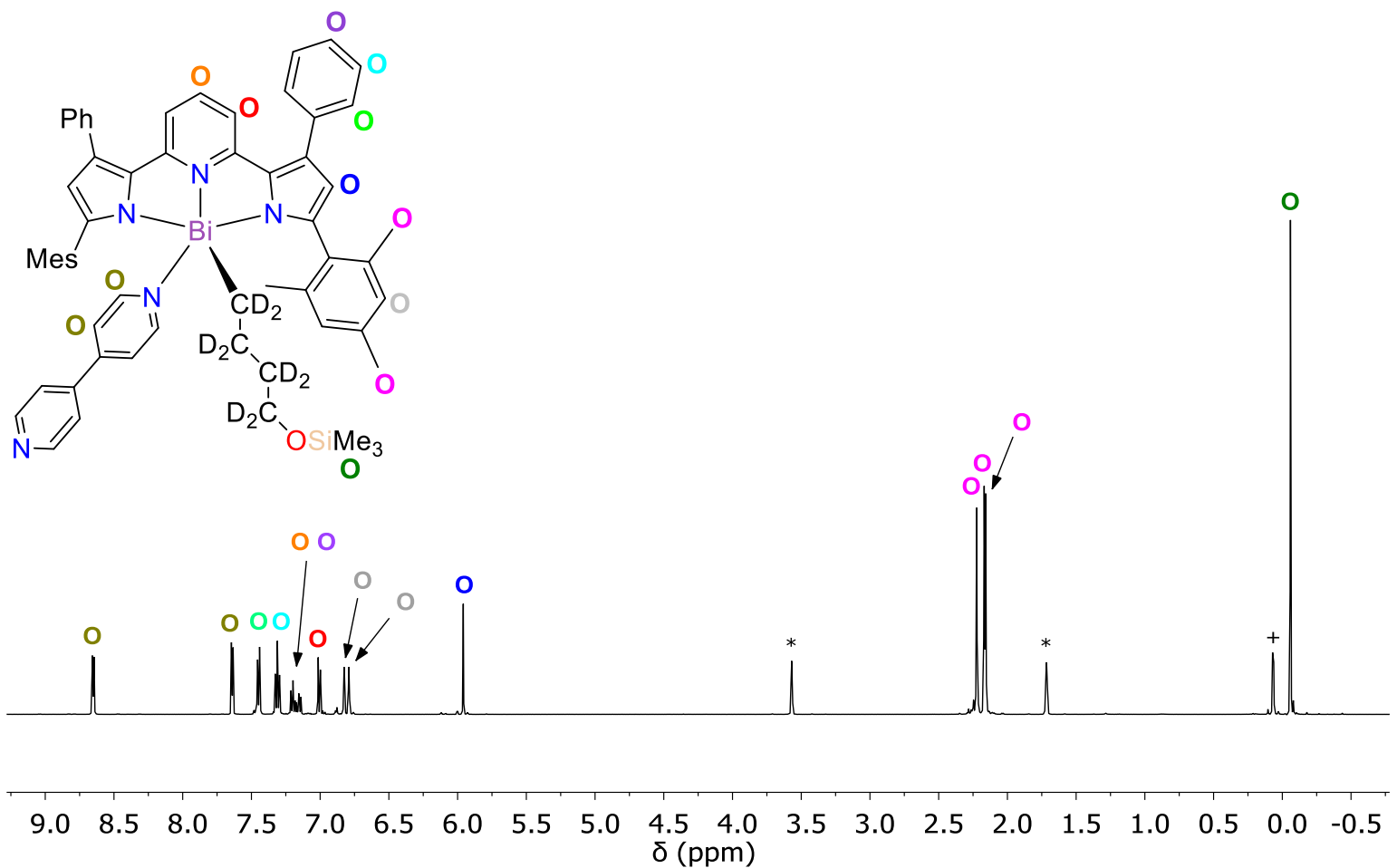

Figure S36: ${ }^{1} \mathrm{H}$ NMR spectrum $\left(\mathrm{C}_{4} \mathrm{D}_{8} \mathrm{O}, 500 \mathrm{MHz}, 298 \mathrm{~K}\right)$ of $\left({ }^{\mathrm{Mes}, \mathrm{Ph}} \mathrm{L}\right) \mathrm{Bi}\left(\left\{\mathrm{CD}_{2}\right\}_{4} \mathrm{OSSiMe}_{3}\right)(4,4$ '-bipy $)$ $\left(\mathbf{1 4} \cdot \mathbf{4 , 4}\right.$ '-bipy). + denotes residual $\mathrm{Me}_{3} \mathrm{SiBr}$. ${ }^{*}$ denotes residual protio solvent fraction of $\mathrm{C}_{4} \mathrm{D}_{8} \mathrm{O}$.

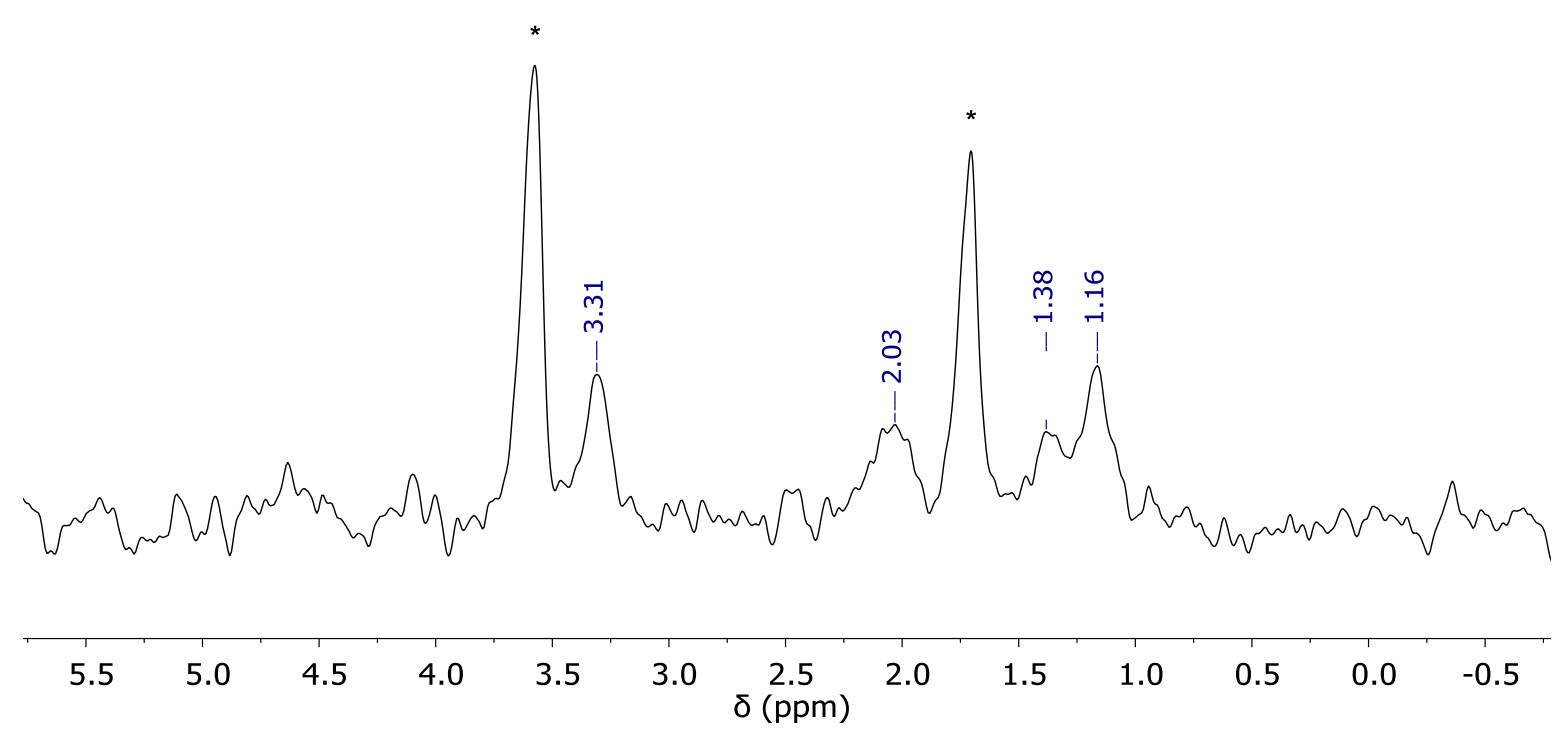

Figure S37: ${ }^{2} \mathrm{H}$ NMR spectrum $\left(\mathrm{C}_{4} \mathrm{H}_{8} \mathrm{O}, 76.74 \mathrm{MHz}, 298 \mathrm{~K}\right)$ of $\left({ }^{\mathrm{Mes}, \mathrm{Ph}} \mathrm{L}\right) \mathrm{Bi}\left(\left\{\mathrm{CD}_{2}\right\}_{4} \mathrm{OSiMe}_{3}\right)\left(4,4\right.$ '-bipy $\left.^{\prime}\right)$ (14·4,4'-bipy). * denotes $\mathrm{C}_{4} \mathrm{D}_{8} \mathrm{O}$. 


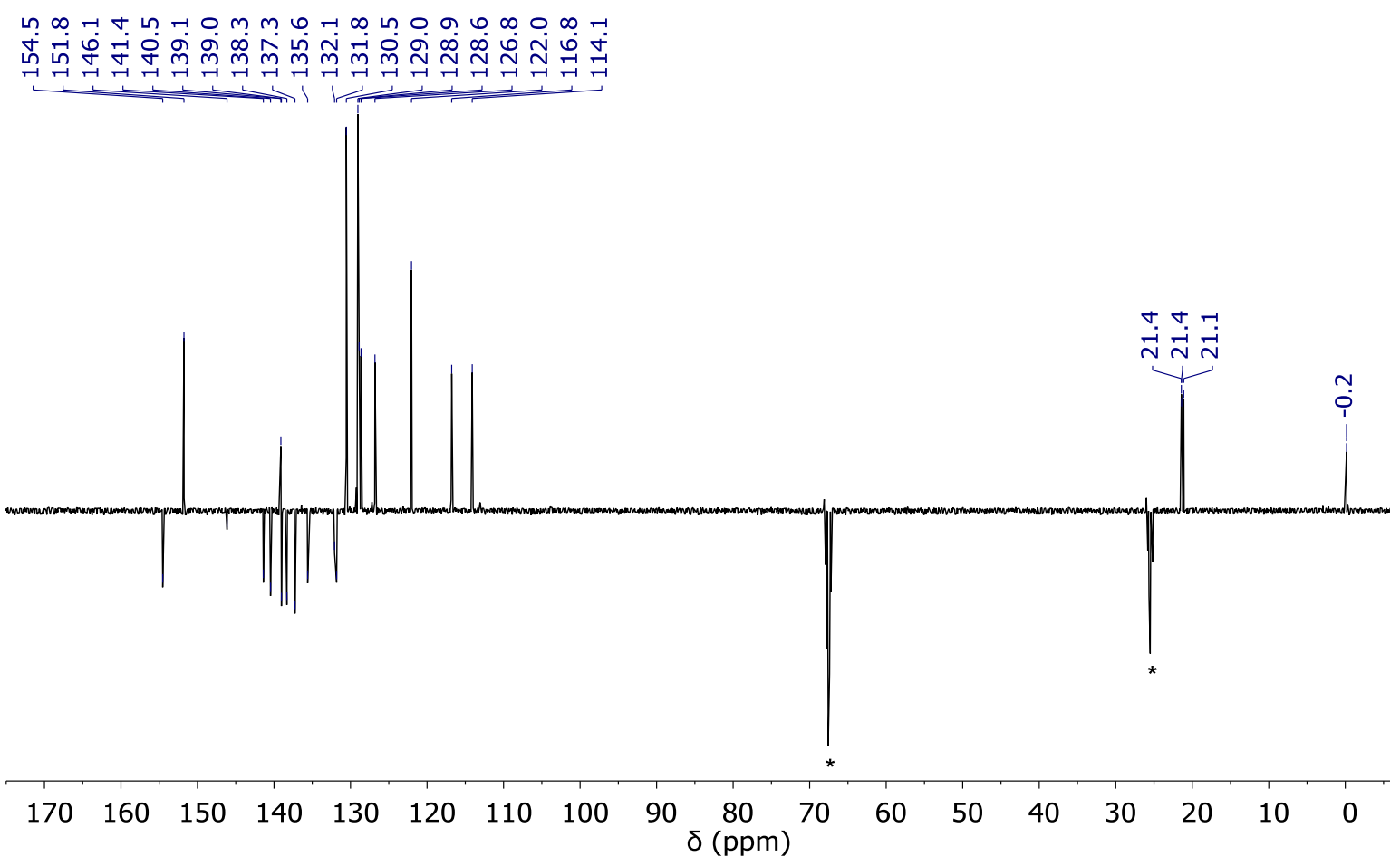

Figure S38: ${ }^{13} \mathrm{C}\left\{{ }^{1} \mathrm{H}\right\}$ APT (attached proton test) NMR spectrum $\left(\mathrm{C}_{4} \mathrm{D}_{8} \mathrm{O}, 125 \mathrm{MHz}, 298 \mathrm{~K}\right)$ of ( $\left.{ }^{\text {Mes,Ph }} \mathrm{L}\right) \mathrm{Bi}\left(\left\{\mathrm{CD}_{2}\right\}_{4} \mathrm{OSiMe}\right)\left(4,4\right.$ '-bipy) $\left(\mathbf{1 4} \cdot \mathbf{4 , 4}\right.$ '-bipy). * denotes $\mathrm{C}_{4} \mathrm{D}_{8} \mathrm{O}$. 


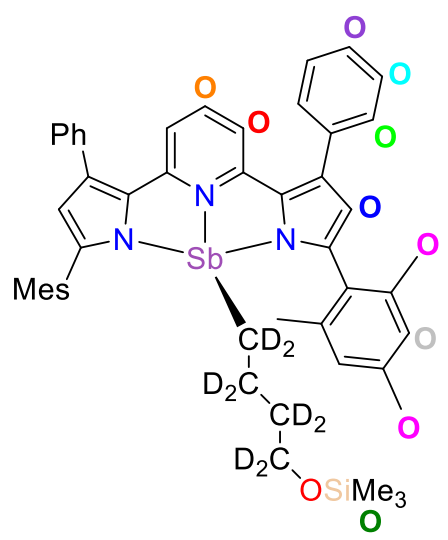

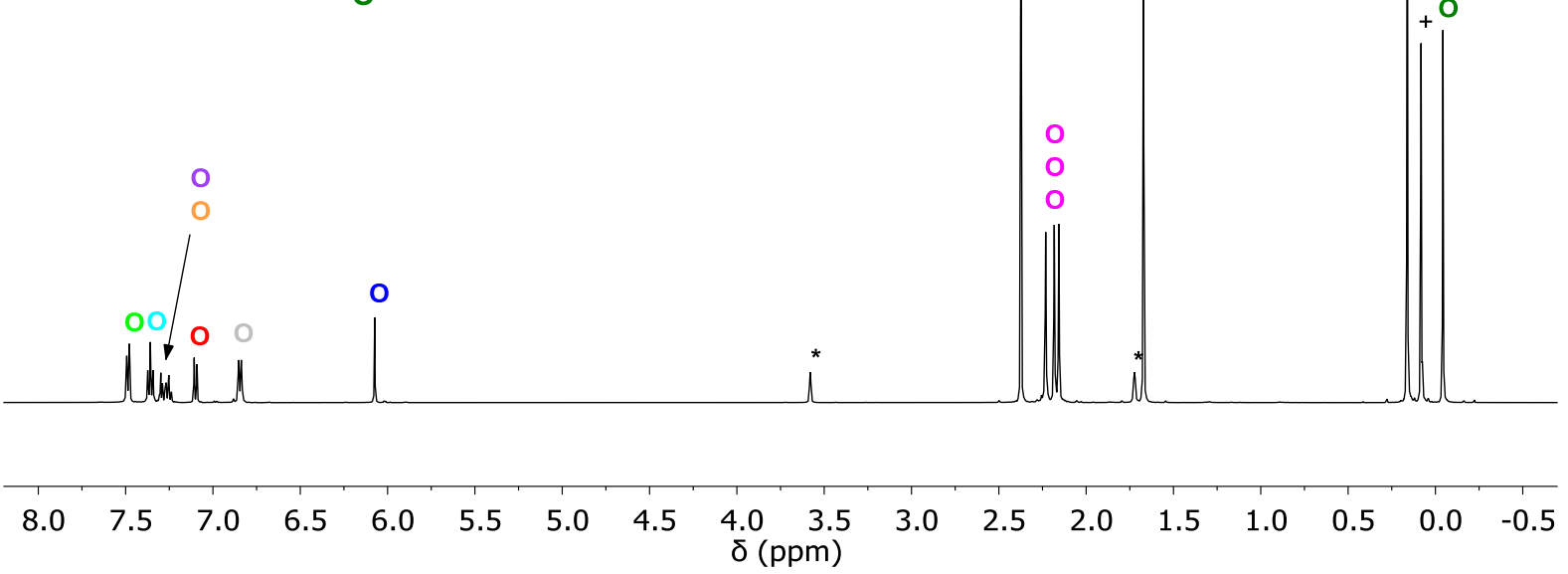

Figure S39: ${ }^{1} \mathrm{H}$ NMR spectrum $\left(\mathrm{C}_{6} \mathrm{D}_{6}, 500 \mathrm{MHz}, 298 \mathrm{~K}\right)$ of $\left({ }^{\mathrm{Mes}, \mathrm{Ph}} \mathrm{L}\right) \mathrm{Sb}\left(\left\{\mathrm{CD}_{2}\right\}_{4} \mathrm{OSiMe}_{3}\right)(\mathbf{1 5})$ prepared in situ using excess reductant $\mathbf{F}$. \# denotes tetramethyl pyrazine, + denotes residual $\mathrm{Me}_{3} \mathrm{SiI}$, $\wedge$ denotes reductant $\mathbf{F}$. $*$ denotes residual protio solvent fraction of $\mathrm{C}_{6} \mathrm{D}_{6}$.

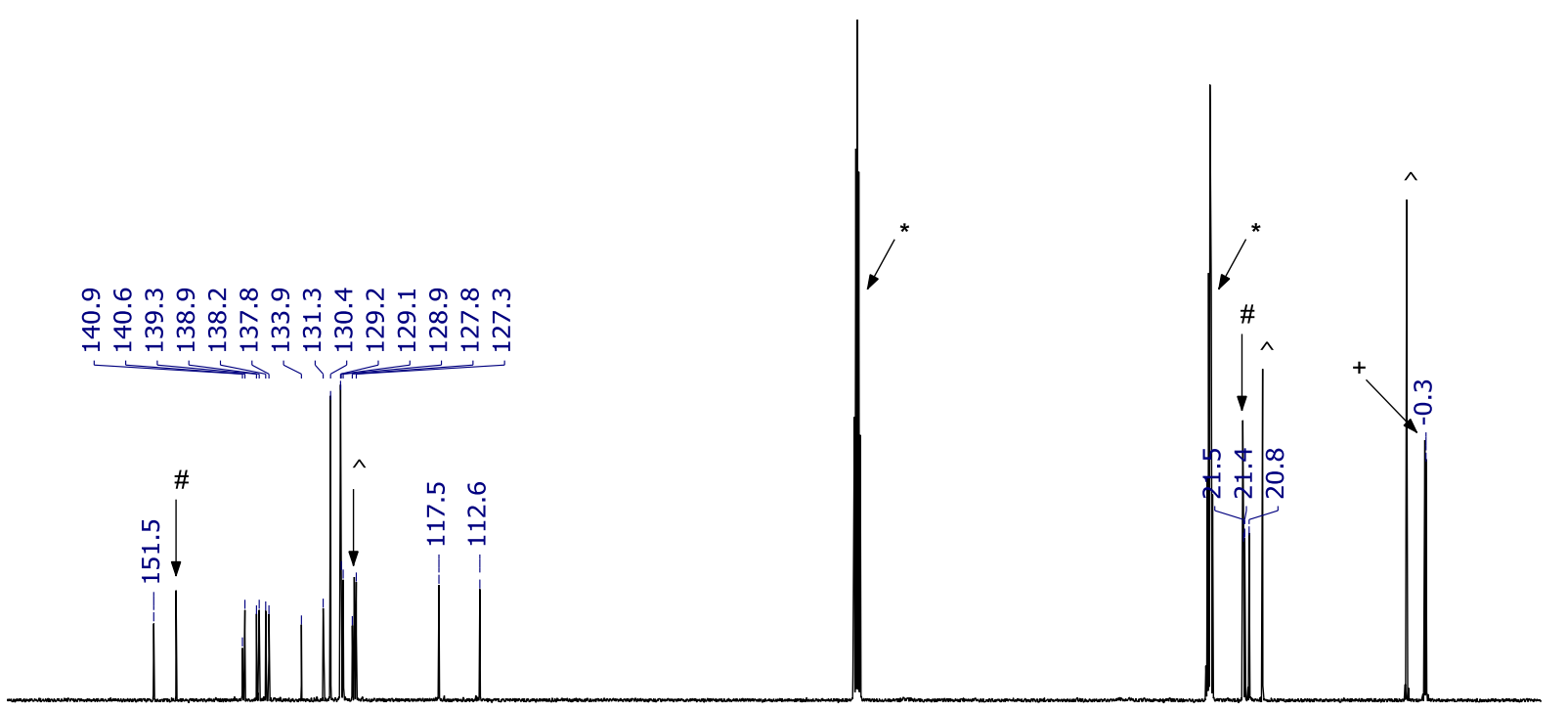

$\begin{array}{llllllllllllllllll}160 & 150 & 140 & 130 & 120 & 110 & 100 & 90 & 80 & 70 & 60 & 50 & 40 & 30 & 20 & 10 & 0 & -10\end{array}$

Figure S40: ${ }^{13} \mathrm{C}\left\{{ }^{1} \mathrm{H}\right\}$ NMR spectrum $\left(\mathrm{C}_{6} \mathrm{D}_{6}, 125 \mathrm{MHz}, 298 \mathrm{~K}\right)$ of $\left({ }^{\mathrm{Mes}, \mathrm{Ph}} \mathrm{L}\right) \mathrm{Sb}\left(\left\{\mathrm{CD}_{2}\right\}_{4} \mathrm{OSiMe}_{3}\right)(\mathbf{1 5})$ prepared in situ using excess reductant $\mathbf{F}$. \# denotes tetramethyl pyrazine, + denotes residual $\mathrm{Me}_{3} \mathrm{SiI}$, $\wedge$ denotes reductant $\mathbf{F}$. $*$ denotes $\mathrm{C}_{6} \mathrm{D}_{6}$. 

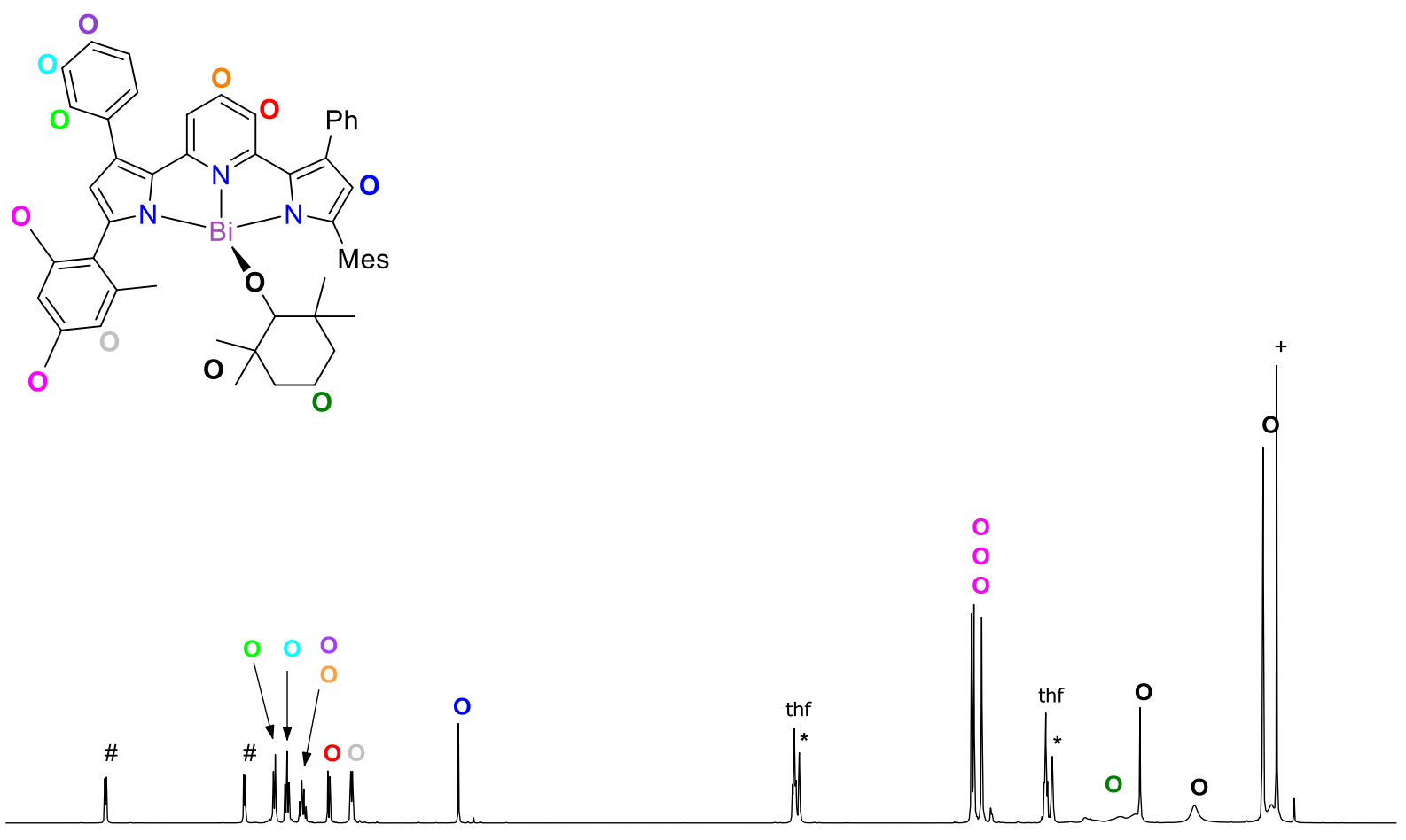

$\begin{array}{llllllllllllllllllll}9.0 & 8.5 & 8.0 & 7.5 & 7.0 & 6.5 & 6.0 & 5.5 & 5.0 & \begin{array}{c}4.5 \\ \delta(\mathrm{ppm})\end{array} & 4.0 & 3.5 & 3.0 & 2.5 & 2.0 & 1.5 & 1.0 & 0.5 & 0.0 & -0.5\end{array}$

Figure S41: ${ }^{1} \mathrm{H}$ NMR spectrum $\left(\mathrm{C}_{4} \mathrm{D}_{8} \mathrm{O}, 500 \mathrm{MHz}, 298 \mathrm{~K}\right)$ of $\left({ }^{\mathrm{Mes}, \mathrm{Ph}} \mathrm{L}\right) \mathrm{Bi}(\mathrm{OTEMP})(\mathbf{1 6})$ generated in situ by reaction of $\mathbf{4} \cdot \mathbf{t h f}_{2}$ with reductant $\mathbf{F}$ and TEMPO. \# denotes 4,4'-bipy. + denotes $\mathrm{Me}_{3} \mathrm{SiI}$. $*$ denotes residual protio solvent fraction of $\mathrm{C}_{4} \mathrm{D}_{8} \mathrm{O}$.

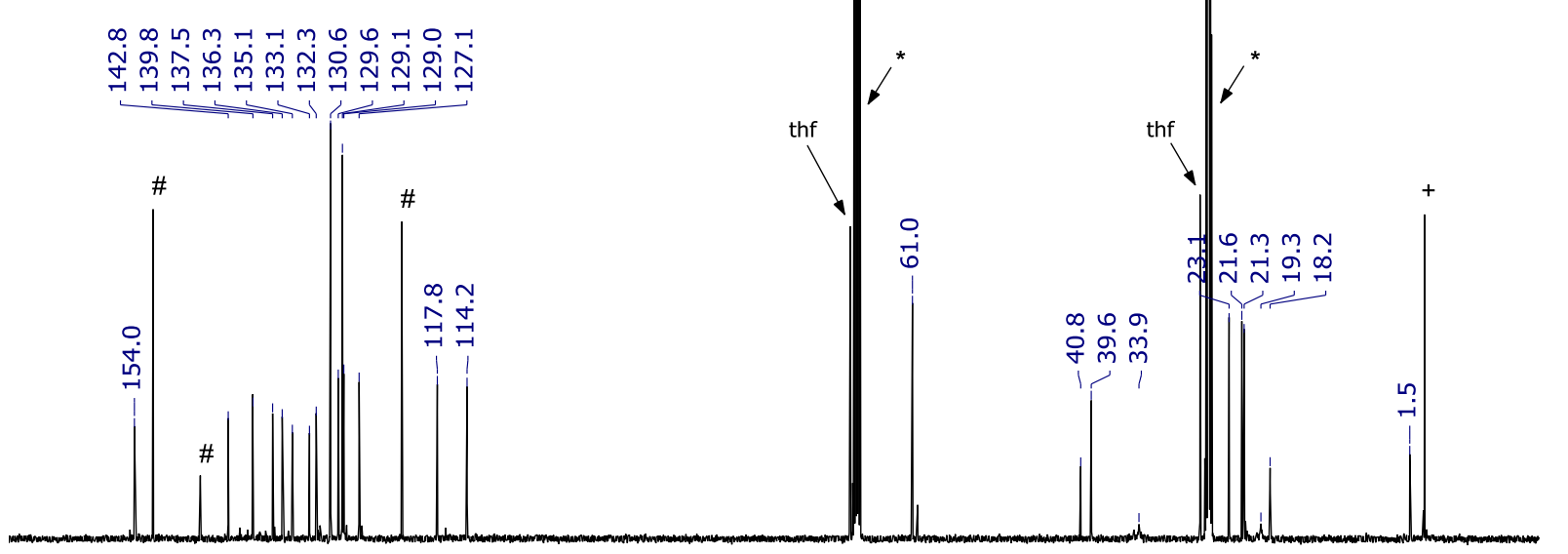

$\begin{array}{llllllllllllllllll}160 & 150 & 140 & 130 & 120 & 110 & 100 & 90 & \underset{\delta(\mathrm{ppm})}{80} \mathbf{7 0} & 60 & 50 & 40 & 30 & 20 & 10 & 0 & -10\end{array}$

Figure S42: ${ }^{13} \mathrm{C}\left\{{ }^{1} \mathrm{H}\right\}$ NMR spectrum $\left(\mathrm{C}_{4} \mathrm{D}_{8} \mathrm{O}, 125 \mathrm{MHz}, 298 \mathrm{~K}\right)$ of $\left({ }^{\mathrm{Mes}, \mathrm{Ph}} \mathrm{L}\right) \mathrm{Bi}(\mathrm{OTEMP})(\mathbf{1 6})$ generated in situ by reaction of $\mathbf{4} \cdot \mathbf{t h f}_{\mathbf{2}}$ with reductant $\mathbf{F}$ and TEMPO. \# denotes 4,4'-bipy. + denotes $\mathrm{Me}_{3} \mathrm{SiI}$. * denotes residual protio solvent fraction of $\mathrm{C}_{4} \mathrm{D}_{8} \mathrm{O}$. 


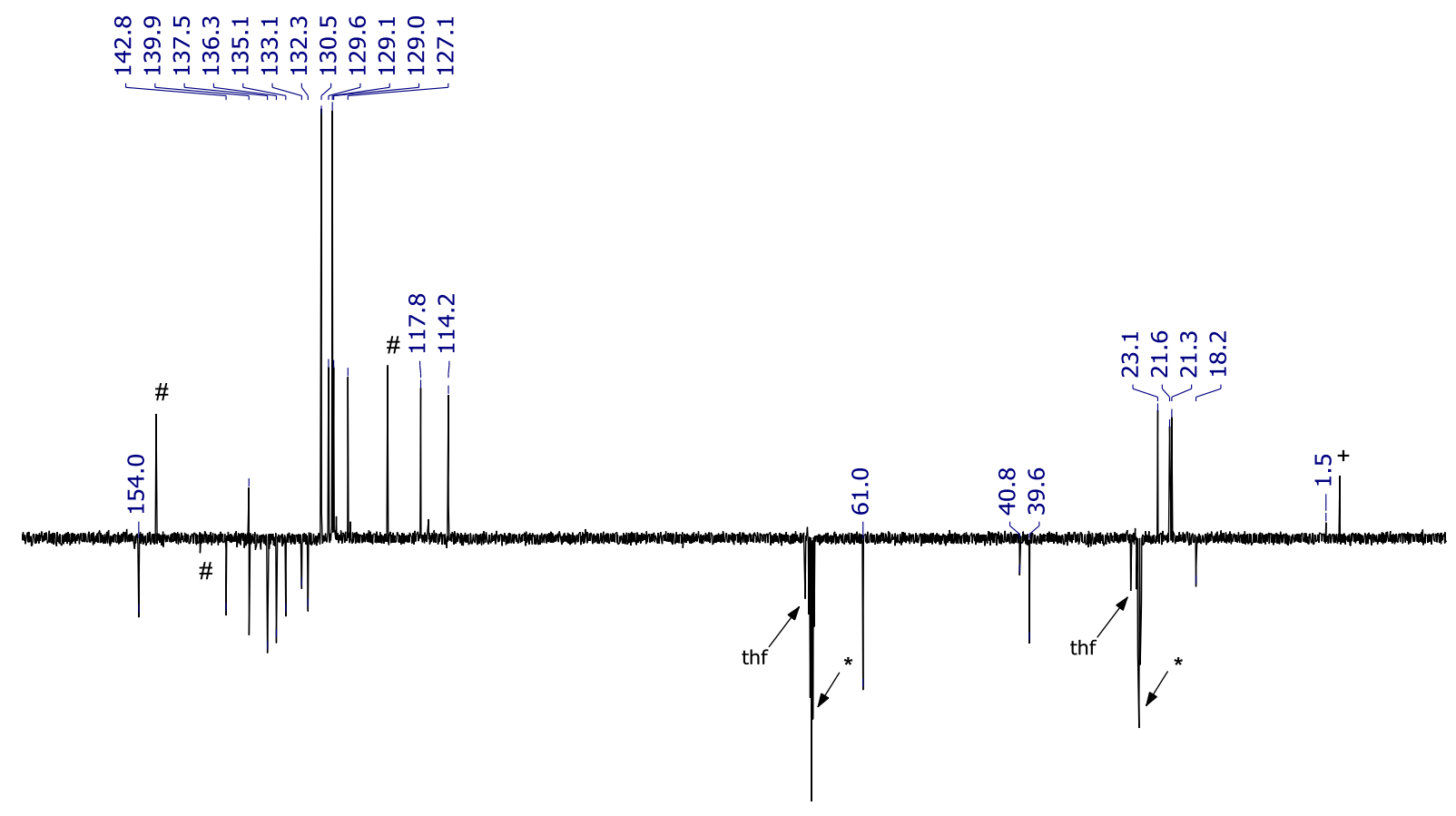

$\begin{array}{llllllllllllllllll}160 & 150 & 140 & 130 & 120 & 110 & 100 & 90 & \begin{array}{c}80 \\ \delta(\mathrm{ppm})\end{array} & 60 & 50 & 40 & 30 & 20 & 10 & 0 & -10\end{array}$

Figure S43: ${ }^{13} \mathrm{C}\left\{{ }^{1} \mathrm{H}\right\}$ APT (attached proton test) NMR spectrum $\left(\mathrm{C}_{4} \mathrm{D}_{8} \mathrm{O}, 125 \mathrm{MHz}, 298 \mathrm{~K}\right)$ of $\left({ }^{\mathrm{Mes}, \mathrm{Ph}} \mathrm{L}\right) \mathrm{Bi}(\mathrm{OTEMP})(\mathbf{1 6})$ generated in situ by reaction of $\mathbf{4} \cdot \mathbf{t h \mathbf { f } _ { 2 }}$ with reductant $\mathbf{F}$ and TEMPO. \# denotes 4,4'-bipy. + denotes $\mathrm{Me}_{3}$ SiI. * denotes $\mathrm{C}_{4} \mathrm{D}_{8} \mathrm{O}$. The broad resonances in Figure $\mathrm{S} 42$ could not be observed in this spectrum. 


\section{Crystallographic data}

Crystals were mounted on MiTeGen MicroMounts using perfluoropolyether oil and rapidly transferred to a goniometer head on a diffractometer fitted with an Oxford Cryosystems Cryostream open-flow nitrogen cooling device. ${ }^{8}$ Data collections were carried out at $150 \mathrm{~K}$ using an Oxford Diffraction Supernova diffractometer using mirror-monochromated $\mathrm{Cu} K \alpha$ radiation $(\lambda=1.54178 \AA)$ and data were processed using CrysalisPro ${ }^{9}$ The structures were solved using direct methods (SIR-92) ${ }^{10}$ or a charge flipping algorithm (SUPERFLIP) ${ }^{11}$ and refined by full-matrix least-squares procedures using the WinGX software suite. ${ }^{12}$ Solid state structural data have been deposited in the Cambridge Crystallographic Data Centre (1944234-1944250). 
Table S1: Selected experimental crystallographic data.

\begin{tabular}{|c|c|c|c|}
\hline Complex & $\mathbf{H}_{2}{ }^{\text {Mes,Ph }} \mathbf{L}$ & $\mathbf{H}_{2}{ }^{\text {Mes,Ph}} \mathbf{L} \cdot \mathbf{E t}_{2} \mathbf{O}$ & $\begin{array}{c}\mathbf{K}_{2}{ }^{\mathrm{Mes}, \mathrm{Ph}} \mathbf{L}\left(\mathbf{E t}_{2} \mathbf{O}\right)_{4} \\
\left(\mathbf{1} \cdot\left(\mathbf{E t}_{2} \mathbf{O}\right)_{4}\right)\end{array}$ \\
\hline \multicolumn{4}{|c|}{ Crystal data } \\
\hline Chemical formula & $\mathrm{C}_{86} \mathrm{H}_{78} \mathrm{~N}_{6}$ & $\mathrm{C}_{43} \mathrm{H}_{39} \mathrm{~N}_{3} \cdot \mathrm{C}_{4} \mathrm{H}_{10} \mathrm{O}$ & $\mathrm{C}_{59} \mathrm{H}_{77} \mathrm{~K}_{2} \mathrm{~N}_{3} \mathrm{O}_{4}$ \\
\hline$M_{\mathrm{r}}$ & 1195.54 & 671.89 & 970.43 \\
\hline Crystal system, space group & Triclinic, $P^{-} 1$ & Monoclinic, $P 2_{1} / n$ & Monoclinic, $P 2_{1}$ \\
\hline Temperature (K) & 150 & 150 & 150 \\
\hline$a, b, c(\AA)$ & $15.1415(6), 16.0790(7), 17.5721(6)$ & $16.9832(3), 12.3124(2), 18.5723(4)$ & $11.7836(2), 12.0315$ (2), 20.1918 (4) \\
\hline$\alpha, \beta, \gamma\left(^{\circ}\right)$ & $83.950(3), 68.344(3), 66.264(4)$ & $90,90.712(2), 90$ & $90,101.485(2), 90$ \\
\hline$V\left(\AA^{3}\right)$ & $3635.1(3)$ & $3883.24(13)$ & $2805.36(9)$ \\
\hline$Z$ & 2 & 4 & 2 \\
\hline Radiation type & $\mathrm{Cu} K \alpha$ & $\mathrm{Cu} K \alpha$ & $\mathrm{Cu} K \alpha$ \\
\hline$\mu\left(\mathrm{mm}^{-1}\right)$ & 0.49 & 0.52 & 1.85 \\
\hline \multirow[t]{2}{*}{ Crystal size (mm) } & $0.26 \times 0.16 \times 0.13$ & $0.35 \times 0.060 \times 0.040$ & $0.32 \times 0.11 \times 0.06$ \\
\hline & \multicolumn{2}{|c|}{ Data Collection } & \\
\hline Diffractometer & $\begin{array}{l}\text { SuperNova, Dual, } \mathrm{Cu} \text { at zero, Atlas } \\
\text { diffractometer }\end{array}$ & $\begin{array}{l}\text { SuperNova, Dual, } \mathrm{Cu} \text { at zero, Atlas } \\
\text { diffractometer }\end{array}$ & $\begin{array}{l}\text { SuperNova, Dual, } \mathrm{Cu} \text { at zero, Atlas } \\
\text { diffractometer }\end{array}$ \\
\hline Absorption correction & $\begin{array}{l}\text { Multi-scan } \\
\text { CrysAlis PRO 1.171.39.46 (Rigaku } \\
\text { Oxford Diffraction, 2018) Empirical } \\
\text { absorption correction using spherical } \\
\text { harmonics, implemented in SCALE3 }\end{array}$ & $\begin{array}{c}\text { Gaussian } \\
\text { CrysAlis PRO 1.171.39.46 (Rigaku } \\
\text { Oxford Diffraction, 2018) Numerical } \\
\text { absorption correction based on gaussian } \\
\text { integration over a multifaceted crystal }\end{array}$ & $\begin{array}{c}\text { Gaussian } \\
\text { CrysAlis PRO 1.171.39.46 (Rigaku } \\
\text { Oxford Diffraction, 2018) Numerical } \\
\text { absorption correction based on gaussian } \\
\text { integration over a multifaceted crystal }\end{array}$ \\
\hline
\end{tabular}


ABSPACK scaling algorithm.

$$
T_{\min }, T_{\max }
$$

No. of measured, independent and observed $[I>2 \sigma(I)]$ reflections

$$
R_{\text {int }}
$$

$$
R\left[F^{2}>2 \sigma\left(F^{2}\right)\right], w R\left(F^{2}\right), S
$$

No. of reflections

No. of parameters

No. of restraints

$$
(\Delta / \sigma)_{\max }
$$

$\Delta \rho_{\max }, \Delta \rho_{\min }\left(\mathrm{e} \AA^{-3}\right)$

Absolute structure

Absolute structure parameter model Empirical absorption correction using spherical harmonics, implemented

in SCALE3 ABSPACK scaling algorithm.

$0.849,1.000$

$39226,14825,12392$

0.035

$0.052,0.152,1.03$

14825

858

28

0.001

$0.27,-0.26$
$0.721,1.000$

$23051,7743,6236$

0.030

\section{Refinement}

$0.043,0.123,1.04$

7743

476

0

0.001

$0.56,-0.23$ model Empirical absorption correction

using spherical harmonics, implemented in SCALE3 ABSPACK scaling algorithm

\section{$0.654,1.000$}

19744, 10492, 9411

0.027

$0.042,0.116,1.04$

10492

645

21

0.002

$0.41,-0.21$

Flack x determined using 3732 quotients $[(\mathrm{I}+)-(\mathrm{I}-)] /[(\mathrm{I}+)+(\mathrm{I}-)]$ (Parsons, Flack and Wagner, Acta Cryst. B69 (2013) 249259).

$-0.013(5)$ 


\begin{tabular}{|c|c|c|c|}
\hline Complex & $\begin{array}{c}\mathrm{K}_{2}^{\mathrm{Mes}, \mathrm{Ph}} \mathbf{L}(\mathrm{OTEMP})_{2}\left(\mathrm{Et}_{2} \mathrm{O}\right)_{2} \\
\left(\mathbf{1} \cdot(\mathrm{OTEMP})_{2}\left(\mathrm{Et}_{2} \mathbf{O}\right)_{2}\right)\end{array}$ & $\begin{array}{c}\left({ }^{\mathrm{t}_{\mathrm{Bu}}, \mathrm{t}_{\mathrm{Bu}} \mathrm{L}}\right) \mathrm{BiI} \\
(2)\end{array}$ & $\begin{array}{c}\left({ }^{\mathrm{t}_{\mathrm{Bu}},{ }_{\mathrm{Bu}} \mathrm{L}}\right) \mathrm{BiCl} \\
(\mathbf{3})\end{array}$ \\
\hline
\end{tabular}

Crystal data

Chemical formula

$$
M_{\text {r }}
$$

Crystal system, space group

Temperature (K)

$$
\begin{gathered}
a, b, c(\AA) \\
\alpha, \beta, \gamma\left(^{\circ}\right) \\
V\left(\AA^{3}\right) \\
Z
\end{gathered}
$$

Radiation type

$$
\mu\left(\mathrm{mm}^{-1}\right)
$$

Crystal size (mm)

Diffractometer

Absorption correction

$\mathrm{C}_{69} \mathrm{H}_{93} \mathrm{~K}_{2} \mathrm{~N}_{5} \mathrm{O}_{4}$

$$
1134.68
$$

Triclinic, $P^{-} 1$

150

11.6737 (3), 12.0499 (3), 23.7808 (5)

97.947 (2), 91.587 (2), 99.634 (2)

3261.89 (14)

2

$\mathrm{Cu} K \alpha$

1.66

$0.18 \times 0.12 \times 0.07$

$\mathrm{C}_{29} \mathrm{H}_{41} \mathrm{BiIN}_{3}$

767.53

Monoclinic, $C 2$

150

25.4591 (6), 6.1761 (2), 18.4274 (4)

90, 94.056 (2), 90

$2890.23(13)$

4

$\mathrm{Cu} K \alpha$

20.50

$0.08 \times 0.15 \times 0.17$

\section{Data Collection}

SuperNova, Dual, $\mathrm{Cu}$ at zero, Atlas diffractometer

\section{Gaussian}

CrysAlis PRO 1.171.39.46 (Rigaku Oxford Diffraction, 2018) Numerical absorption correction based on gaussian integration over a multifaceted crystal model Empirical absorption correction using spherical harmonics, implemented
SuperNova, Dual, $\mathrm{Cu}$ at zero, Atlas diffractometer

\section{Multi-scan}

CrysAlis PRO 1.171.39.46 (Rigaku Oxford Diffraction, 2018) Empirical absorption correction using spherical harmonics, implemented in SCALE3 ABSPACK scaling algorithm.

\section{$\mathrm{C}_{33} \mathrm{H}_{51} \mathrm{BiClN}_{3} \mathrm{O}$}

750.19

Monoclinic, $P 2{ }_{1} / n$

150

$10.2780(1), 18.9853$ (1), 17.4369 (1)

$90,97.563(1), 90$

$3372.88(4)$

$\mathrm{Cu} K \alpha$

11.19

$0.20 \times 0.13 \times 0.08$
SuperNova, Dual, $\mathrm{Cu}$ at zero, Atlas diffractometer

Multi-scan

CrysAlis PRO 1.171.38.41 (Rigaku Oxford Diffraction, 2015) Empirical absorption correction using spherical harmonics, implemented in SCALE3 ABSPACK scaling algorithm. 
in SCALE3 ABSPACK scaling algorithm.

$$
T_{\min }, T_{\max }
$$

$0.794,1.000$

$55192,13321,11040$

No. of measured, independent and
observed $[I>2 \sigma(I)]$ reflections

$R_{\text {int }}$

$$
R\left[F^{2}>2 \sigma\left(F^{2}\right)\right], w R\left(F^{2}\right), S
$$

No. of reflections

No. of parameters

No. of restraints

$(\Delta / \sigma)_{\max }$

$\Delta \rho_{\max }, \Delta \rho_{\min }\left(\mathrm{e} \AA^{-3}\right)$

$0.037,0.101,1.02$

13321

739

0

0.001

$0.30,-0.22$
$0.598,0.922$

13884, 5740, 5430

0.049
$0.463,1.000$

$68029,6898,6771$

0.032

Refinement
$0.017,0.041,1.09$

6898

6366

21

0.007

$0.48,-1.07$ 


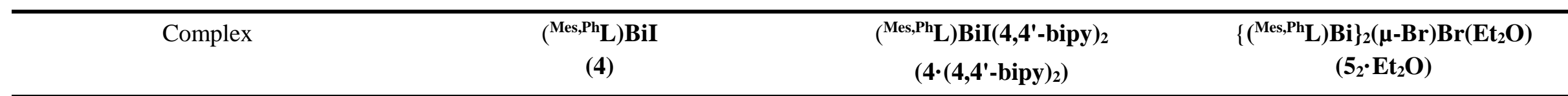

Chemical formula
$M_{\mathrm{r}}$
Crystal system, space
Temperature $(\mathrm{K})$
$a, b, c(\AA)$
$\alpha, \beta, \gamma\left(^{\circ}\right)$
$V\left(\AA^{3}\right)$
$Z$

Radiation type

$\mu\left(\mathrm{mm}^{-1}\right)$

Crystal size (mm)

Diffractometer

Absorption correction

\section{Crystal data}

$\mathrm{C}_{43} \mathrm{H}_{37} \mathrm{BiIN}_{3}$

931.63

Triclinic, $P^{-} 1$

150

$\mathrm{C}_{63} \mathrm{H}_{53} \mathrm{BiIN}_{7}$

1244.00

Triclinic, $P^{-} 1$

150
$\mathrm{C}_{47} \mathrm{H}_{47} \mathrm{BiBrN}_{3} \mathrm{O} \cdot \mathrm{C}_{43} \mathrm{H}_{37} \mathrm{BiBrN}_{3}$

\subsection{1}

Monoclinic, $P 2_{1} / n$

150

11.9065 (3), 12.7479 (4), 13.3204 (5) 13.8997 (4), 15.1699 (4), 16.5864 (5) 11.7809 (1), 25.0513 (2), 26.1063 (2)

$97.020(3), 112.250(3), 97.204(2) \quad 101.438(2), 103.570(2), 110.332(2)$

90, 91.835 (1), 90

$1824.51(11)$

2

$\mathrm{Cu} K \alpha$

16.38

$0.25 \times 0.13 \times 0.06$
$3033.96(16)$

2

$\mathrm{Cu} K \alpha$

10.01

$0.15 \times 0.12 \times 0.08$
7700.72 (11)

4

$\mathrm{Cu} K \alpha$

10.48

$0.23 \times 0.15 \times 0.04$

\section{Data Collection}

SuperNova, Dual, $\mathrm{Cu}$ at zero, Atlas diffractometer

Gaussian
CrysAlis PRO 1.171.38.41 (Rigaku
Oxford Diffraction, 2015) Numerical
absorption correction based on gaussian
integration over a multifaceted crystal
model Empirical absorption correction
using spherical harmonics, implemented

SuperNova, Dual, $\mathrm{Cu}$ at zero, Atlas diffractometer

Gaussian CrysAlis PRO 1.171.39.46 (Rigaku Oxford Diffraction, 2018) Numerical absorption correction based on gaussian integration over a multifaceted crystal model Empirical absorption correction using spherical harmonics, implemented
SuperNova, Dual, $\mathrm{Cu}$ at zero, Atlas diffractometer

\section{Multi-scan}

CrysAlis PRO 1.171.39.46 (Rigaku Oxford Diffraction, 2018) Empirical absorption correction using spherical harmonics, implemented in SCALE3

ABSPACK scaling algorithm. 
in SCALE3 ABSPACK scaling algorithm.

$$
T_{\min }, T_{\max }
$$

$0.302,0.657$

No. of measured, independent and observed $[I>2 \sigma(I)]$ reflections

$$
R_{\text {int }}
$$

$R\left[F^{2}>2 \sigma\left(F^{2}\right)\right], w R\left(F^{2}\right), S$

No. of reflections

No. of parameters

No. of restraints

$(\Delta / \sigma)_{\max }$

$\Delta \rho_{\max }, \Delta \rho_{\min }\left(\mathrm{e} \AA^{-3}\right)$
$17665,7443,7235$

0.023

$0.020,0.053,1.08$

7443

439

0

0.02

$0.78,-1.60$ in SCALE3 ABSPACK scaling

algorithm.

$$
0.589,1.000
$$

$32764,12369,11457$

0.023

\section{Refinement}

$0.022,0.056,0.98$

12369

655

0.014

$0.61,-1.09$
0.059

$0.404,1.000$

$105272,15763,13837$

$0.035,0.093,1.03$

15763

923

51

0.002

$2.10,-1.62$ 


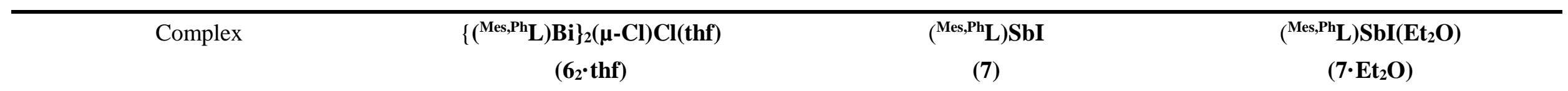

$\left(6_{2} \cdot\right.$ thf $)$

(7)

$\left(7 \cdot \mathrm{Et}_{2} \mathrm{O}\right)$

Crystal data

Chemical formula

$M_{\mathrm{r}}$

Crystal system, space group

Temperature (K)

$$
\begin{gathered}
a, b, c(\AA) \\
\alpha, \beta, \gamma\left(^{\circ}\right) \\
V\left(\AA^{3}\right) \\
Z
\end{gathered}
$$

Radiation type

$$
\mu\left(\mathrm{mm}^{-1}\right)
$$

Crystal size (mm)

Diffractometer

Absorption correction

\section{$\mathrm{C}_{47} \mathrm{H}_{45} \mathrm{BiClN}_{3} \mathrm{O} \cdot \mathrm{C}_{43} \mathrm{H}_{37} \mathrm{BiClN}_{3}$}

1752.47

Monoclinic, $P 2_{1} / n$

150

11.6992 (2), 24.6234 (5), 26.5187 (6)

90, $91.192(2), 90$

7637.7 (3)

4

$\mathrm{Cu} K \alpha$

9.98

$0.20 \times 0.12 \times 0.010$

$\mathrm{C}_{43} \mathrm{H}_{37} \mathrm{IN}_{3} \mathrm{Sb}$

844.40

Triclinic, $P^{-} 1$

150

11.9206 (4), 12.7600 (3), 13.3362 (4)

96.791 (2), 112.075 (3), 97.302 (2)

$1833.91(10)$

2

$\mathrm{Cu} K \alpha$

12.83

$0.15 \times 0.13 \times 0.02$

\section{Data Collection}

SuperNova, Dual, $\mathrm{Cu}$ at zero, Atlas diffractometer

Gaussian

CrysAlis PRO 1.171.39.46 (Rigaku Oxford Diffraction, 2018) Numerical absorption

correction based on gaussian integration over a multifaceted crystal model Empirical absorption correction using spherical harmonics, implemented in
SuperNova, Dual, $\mathrm{Cu}$ at zero, Atlas diffractometer

Analytical

CrysAlis PRO 1.171.39.46 (Rigaku Oxford Diffraction, 2018) Analytical numeric absorption correction using a multifaceted crystal model based on expressions derived by R.C. Clark \& J.S. Reid. (Clark, R. C. \& Reid, J. S. (1995). Acta Cryst. A51, 887-

\section{$\mathrm{C}_{47} \mathrm{H}_{47} \mathrm{IN}_{3} \mathrm{OSb}$}

918.52

Monoclinic, $P 2{ }_{1} / n$

150

16.3320 (1), 18.1476 (2), 29.5306 (3)

90, $91.672(1), 90$

8748.75 (14)

8

$\mathrm{Cu} K \alpha$

10.82

$0.34 \times 0.15 \times 0.13$

SuperNova, Dual, $\mathrm{Cu}$ at zero, Atlas diffractometer

Multi-scan

CrysAlis PRO 1.171.39.46 (Rigaku Oxford Diffraction, 2018) Empirical absorption correction using spherical harmonics, implemented in SCALE3 ABSPACK scaling algorithm. 
SCALE3 ABSPACK scaling algorithm.

$T_{\min }, T_{\max }$

No. of measured, independent and observed $[I>2 \sigma(I)]$ reflections

$R_{\text {int }}$

$R\left[F^{2}>2 \sigma\left(F^{2}\right)\right], w R\left(F^{2}\right), S$

No. of reflections

No. of parameters

No. of restraints

$(\Delta / \sigma)_{\max }$

$\Delta \rho_{\max }, \Delta \rho_{\min }\left(\mathrm{e} \AA^{-3}\right)$

$0.331,1.000$

$56784,15612,11654$

0.064

$0.056,0.113,1.11$
0.034

97) Empirical absorption correction using

spherical harmonics, implemented in

SCALE3 ABSPACK scaling algorithm.

$0.381,1.000$

$0.287,1.000$

$18609,7473,6282$

$53670,17829,14896$

0.044

\section{Refinement}

$0.028,0.068,1.02$

7473

439

0

0.001

$0.90,-0.71$
$0.055,0.152,1.11$

17829

1042

128

0.001

$1.58,-0.83$ 


\begin{tabular}{cc}
\hline Complex & $\left({ }^{\mathrm{t}_{\mathrm{Bu}},{ }^{\mathrm{t}} \mathrm{Bu}} \mathbf{L}\right) \mathrm{Mg}\left(\text { thf }_{2}\right)_{2}$ \\
\end{tabular}

(8)

$\left({ }^{M e s, P h} \mathbf{L}\right) \mathrm{Mg}(\text { thf })_{2}$

(10)
$\mathrm{K}($ thf $)\left[\left({ }^{\mathrm{t}_{\mathrm{Bu}},{ }^{\mathrm{t}} \mathrm{Bu}} \mathrm{L}\right)_{2} \mathrm{Bi}\right]$

(12)

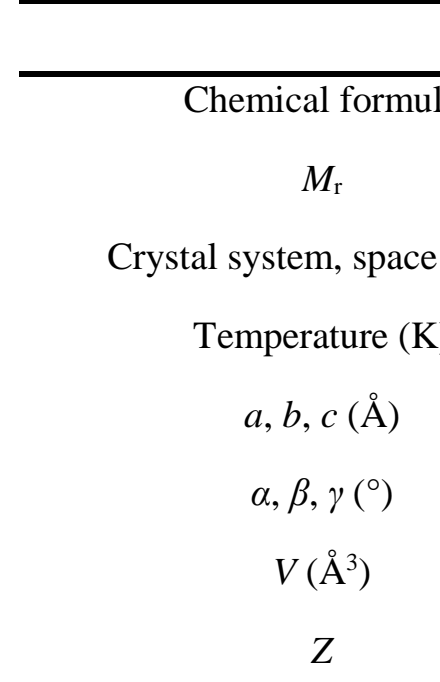

Radiation type

$\mu\left(\mathrm{mm}^{-1}\right)$

Crystal size (mm)

Diffractometer

Absorption correction

$\mathrm{C}_{37} \mathrm{H}_{57} \mathrm{MgN}_{3} \mathrm{O}_{2}$

$$
600.16
$$

Monoclinic, $P 2_{1} / c$

150

16.0433 (5), 23.3591 (6), 19.9971

90, 109.973 (3), 90

7043.3 (4)

8

$\mathrm{Cu} K \alpha$

0.69

$0.20 \times 0.13 \times 0.08$

Crystal data

$\mathrm{C}_{51} \mathrm{H}_{53} \mathrm{MgN}_{3} \mathrm{O}_{2}$

764.27

Triclinic, $P^{-} 1$

150

(5) $11.8608(4), 13.7927(4), 14.6581$ (5)

$69.883(3), 69.320(3), 79.330$ (2)

$$
2101.04 \text { (13) }
$$

2

$\mathrm{Cu} K \alpha$

0.70

$0.25 \times 0.21 \times 0.18$
$\mathrm{C}_{62} \mathrm{H}_{90} \mathrm{BiKN}_{6} \mathrm{O}$

1183.47
Triclinic, $P^{-} 1$

150

13.9047 (2), 22.0342 (2), 23.5618 (3)

104.967 (1), 102.020 (1), 100.552 (1)

$6603.15(15)$

4

$\mathrm{Cu} K \alpha$

6.10

$0.19 \times 0.17 \times 0.13$

\section{Data Collection}

SuperNova, Dual, Cu at zero, Atlas diffractometer

Gaussian
CrysAlis PRO 1.171.39.46 (Rigaku
Oxford Diffraction, 2018) Numerical
absorption correction based on gaussian
integration over a multifaceted crystal
model Empirical absorption correction
using spherical harmonics, implemented

SuperNova, Dual, $\mathrm{Cu}$ at zero, Atlas diffractometer

\section{Multi-scan}

CrysAlis PRO 1.171.39.46 (Rigaku Oxford Diffraction, 2018) Empirical absorption correction using spherical harmonics, implemented in SCALE3 ABSPACK scaling algorithm.
SuperNova, Dual, Cu at zero, Atlas diffractometer

\section{Gaussian}

CrysAlis PRO 1.171.39.46 (Rigaku Oxford Diffraction, 2018) Numerical absorption correction based on gaussian integration over a multifaceted crystal model Empirical absorption correction using spherical harmonics, implemented 
in SCALE3 ABSPACK scaling algorithm

$$
T_{\min }, T_{\max }
$$

No. of measured, independent and observed $[I>2 \sigma(I)]$ reflections

$$
R_{\text {int }}
$$

$$
R\left[F^{2}>2 \sigma\left(F^{2}\right)\right], w R\left(F^{2}\right), S
$$

No. of reflections

No. of parameters

No. of restraints

$$
(\Delta / \sigma)_{\max }
$$

$\Delta \rho_{\max }, \Delta \rho_{\min }\left(\mathrm{e} \AA^{-3}\right)$

$$
0.610,1.000
$$

43663, 14376, 9728

0.062

$0.818,1.000$

25494, 8696, 7898

0.028

\section{Refinement}

$0.057,0.149,1.02$

14376

824

22

$<0.001$

$0.69,-0.43$ in SCALE3 ABSPACK scaling algorithm

$0.481,0.763$

$155664,26992,23466$

0.045

$0.035,0.107,1.05$

26992

1327

0

0.002

$2.85,-1.98$ 


\begin{tabular}{|c|c|c|c|}
\hline Complex & $\begin{array}{c}\left({ }^{\mathrm{Mes}, \mathrm{Ph}} \mathrm{L}\right) \mathrm{Bi}\left\{\left(\mathrm{CH}_{2}\right)_{4} \mathrm{OSiMe}_{3}\right\} \\
(\mathbf{1 4})\end{array}$ & 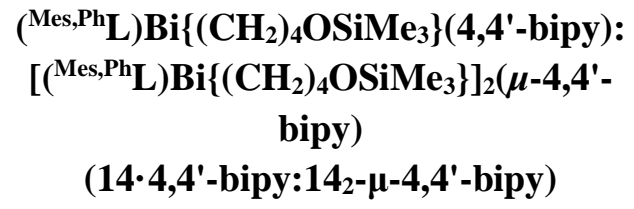 & $\begin{array}{c}\left(\mathrm{Mes}, \mathrm{Ph}^{\mathrm{L}}\right) \mathrm{Sb}\left\{\left(\mathrm{CH}_{2}\right)_{4} \mathrm{OSiMe}_{3}\right\} \\
(\mathbf{1 5})\end{array}$ \\
\hline
\end{tabular}

\section{Crystal data}

\begin{tabular}{|c|c|c|c|}
\hline Chemical formula & $\mathrm{C}_{50} \mathrm{H}_{54} \mathrm{BiN}_{3} \mathrm{OSi}$ & $\begin{array}{c}4\left(\mathrm{C}_{50} \mathrm{H}_{54} \mathrm{BiN}_{3} \mathrm{OSi}\right) \cdot 3\left(\mathrm{C}_{10} \mathrm{H}_{8} \mathrm{~N}_{2}\right) \cdot \mathrm{C}_{6} \mathrm{H}_{18} \mathrm{O} \\
\mathrm{Si}_{2}\end{array}$ & $\mathrm{C}_{50} \mathrm{H}_{54} \mathrm{~N}_{3} \mathrm{OSbSi}$ \\
\hline$M_{\mathrm{r}}$ & 950.03 & 4431.05 & 862.80 \\
\hline Crystal system, space group & Triclinic, $P^{-} 1$ & Triclinic, $P^{-} 1$ & Monoclinic, $I 2 / a$ \\
\hline Temperature (K) & 150 & 150 & 150 \\
\hline$a, b, c(\AA)$ & $12.1668(3), 12.2838(2), 29.6724(4)$ & 12.3975 (2), 19.4059 (3), 22.7888 (4) & $22.5506(1), 15.8427(1), 25.0992(2)$ \\
\hline$\alpha, \beta, \gamma\left({ }^{\circ}\right)$ & $87.621(1), 90.036(2), 85.835(2)$ & $76.048(1), 89.663(1), 85.829(1)$ & 90, $97.398(1), 90$ \\
\hline$V\left(\AA^{3}\right)$ & $4419.12(14)$ & $5306.41(15)$ & $8892.36(10)$ \\
\hline$Z$ & 4 & 1 & 8 \\
\hline Radiation type & $\mathrm{Cu} K \alpha$ & $\mathrm{Cu} K \alpha$ & $\mathrm{Cu} K \alpha$ \\
\hline$\mu\left(\mathrm{mm}^{-1}\right)$ & 8.38 & 7.18 & 5.47 \\
\hline Crystal size (mm) & $0.27 \times 0.13 \times 0.10$ & $0.34 \times 0.08 \times 0.05$ & $0.23 \times 0.13 \times 0.13$ \\
\hline \multicolumn{4}{|c|}{ Data Collection } \\
\hline Diffractometer & $\begin{array}{l}\text { SuperNova, Dual, } \mathrm{Cu} \text { at zero, Atlas } \\
\text { diffractometer }\end{array}$ & $\begin{array}{l}\text { SuperNova, Dual, } \mathrm{Cu} \text { at zero, Atlas } \\
\text { diffractometer }\end{array}$ & $\begin{array}{l}\text { SuperNova, Dual, } \mathrm{Cu} \text { at zero, Atlas } \\
\text { diffractometer }\end{array}$ \\
\hline Absorption correction & $\begin{array}{c}\text { Gaussian } \\
\text { CrysAlis PRO 1.171.39.46 (Rigaku Oxford } \\
\text { Diffraction, 2018) Numerical absorption }\end{array}$ & $\begin{array}{c}\text { Gaussian } \\
\text { CrysAlis PRO 1.171.39.46 (Rigaku Oxford } \\
\text { Diffraction, 2018) Numerical absorption } \\
\text { correction based on gaussian integration }\end{array}$ & $\begin{array}{c}\text { Multi-scan } \\
\text { CrysAlis PRO 1.171.39.46 (Rigaku Oxford } \\
\text { Diffraction, 2018) Empirical absorption } \\
\text { correction using spherical harmonics, }\end{array}$ \\
\hline
\end{tabular}




$$
T_{\min }, T_{\max }
$$

No. of measured, independent and observed $[I>2 \sigma(I)]$ reflections

$R_{\text {int }}$

$$
R\left[F^{2}>2 \sigma\left(F^{2}\right)\right], w R\left(F^{2}\right), S
$$

No. of reflections

No. of parameters

No. of restraints

$$
(\Delta / \sigma)_{\max }
$$

$\Delta \rho_{\max }, \Delta \rho_{\min }\left(\mathrm{e} \AA^{-3}\right)$ correction based on gaussian integration

over a multifaceted crystal model.

Empirical absorption correction using

spherical harmonics, implemented in

SCALE3 ABSPACK scaling algorithm.

$$
0.522,0.843
$$

$79595,17767,15347$

0.050

$0.063,0.180,1.02$

17767

1032

171

0.001

$3.45,-2.48$

over a multifaceted crystal model Empirical absorption correction using spherical harmonics, implemented in SCALE3 ABSPACK scaling algorithm.

$0.167,1.000$

108794, 21687, 19413

0.028

\section{Refinement}

$0.036,0.093,1.04$

21687

1237

0

0.002

$8.42,-1.36$ implemented in SCALE3 ABSPACK

scaling algorithm.

$0.728,1.000$

$52648,9074,8498$

0.024

$0.029,0.083,1.03$

9074

544

0

0.002

$0.71,-0.42$ 


\begin{tabular}{cc}
\hline Complex & $\begin{array}{c}\left({ }^{\text {Mes,Ph }} \mathbf{L}\right) \text { Bi(OTEMP)(thf) } \\
(\mathbf{1 6} \cdot \text { thf })\end{array}$ \\
\hline
\end{tabular}

Crystal data

Chemical formula

$$
M_{\mathrm{r}}
$$

Crystal system, space group

Temperature (K)

$$
\begin{gathered}
a, b, c(\AA) \\
\alpha, \beta, \gamma\left({ }^{\circ}\right) \\
V\left(\AA^{3}\right) \\
Z
\end{gathered}
$$

Radiation type

$$
\mu\left(\mathrm{mm}^{-1}\right)
$$

Crystal size (mm)

Diffractometer

Absorption correction
$\mathrm{C}_{52} \mathrm{H}_{55} \mathrm{BiN}_{4} \mathrm{O} \cdot \mathrm{C}_{4} \mathrm{H}_{8} \mathrm{O}$

1033.08

Monoclinic, $I 2 / a$

150

19.2829 (2), 12.2686 (1), 41.1836 (4)

90, 96.796 (1), 90

$9674.52(16)$

8

$\mathrm{Cu} K \alpha$

7.50

$0.18 \times 0.11 \times 0.06$

\section{Data Collection}

SuperNova, Dual, $\mathrm{Cu}$ at zero, Atlas diffractometer

Multi-scan

CrysAlis PRO, Agilent Technologies, Version 1.171.35.21 (release 20-01-2012

CrysAlis171 .NET) (compiled Jan 23

2012,18:06:46) Empirical absorption

correction using spherical harmonics,

implemented in SCALE3 ABSPACK 
scaling algorithm.

$$
T_{\min }, T_{\max }
$$

No. of measured, independent and observed $[I>2 \sigma(I)]$ reflections

$$
R_{\text {int }}
$$

$$
R\left[F^{2}>2 \sigma\left(F^{2}\right)\right], w R\left(F^{2}\right), S
$$

No. of reflections

No. of parameters

No. of restraints

$$
(\Delta / \sigma)_{\max }
$$

$\Delta \rho_{\max }, \Delta \rho_{\min }\left(\mathrm{e} \AA^{-3}\right)$

$$
0.472,0.821
$$

54982, 9907, 8910

$$
0.033
$$

\section{Refinement}

$0.030,0.075,1.04$

$$
\begin{gathered}
9907 \\
603 \\
28 \\
0.001
\end{gathered}
$$$$
1.58,-1.13
$$

Computer programs: CrysAlis PRO 1.171.39.46 (Rigaku Oxford Diffraction, 2018) CrysAlis PRO, Agilent Technologies, Version 1.171.35.21 (release 2001-2012 CrysAlis171 .NET) (compiled Jan 23 2012,18:06:46), : SUPERFLIP. Palatinus, L.; Chapuis, G. J. Appl. Cryst. 2007, 40, 786-790, : Sir-92. Altomare, A.; Cascarano, G.; Giacovazzo, C.; Guagliardi, A. J. Appl. Cryst. 1994, 27, 435., SIR92 Altomare, A.; Cascarano, G.; Giacovazzo, C.; Guagliardi, A. J. Appl. Cryst. 1994, 27, 435, SHELXL2018/3 (Sheldrick, 2018), SHELXL2014 (Sheldrick, 2014), ORTEP-3 for Windows. Farrugia, L. J. J. Appl. Cryst. 1997, 30, 565. 


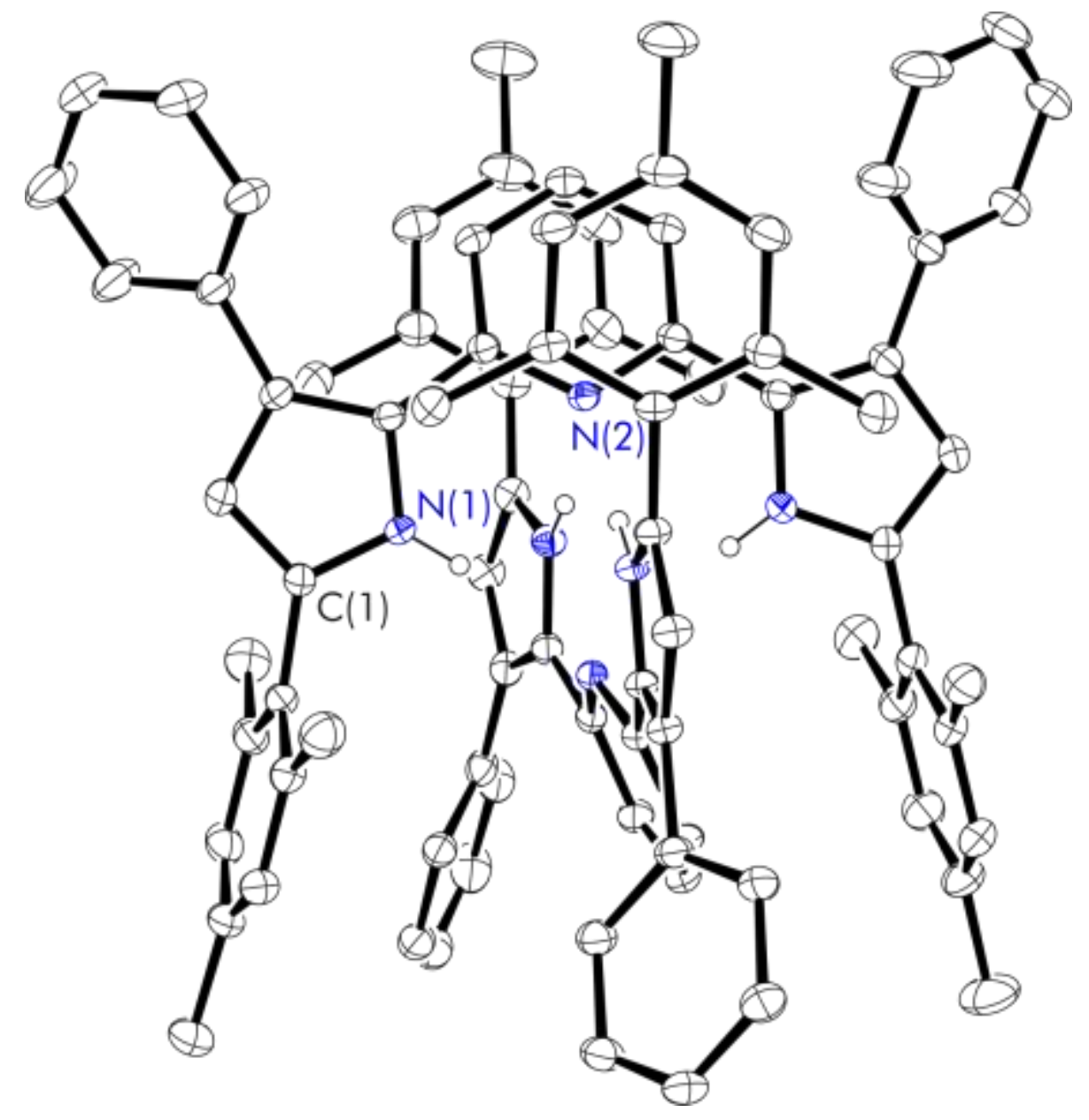

Figure S44: Thermal displacement ellipsoid drawing (30\% probability) of $\mathrm{H}_{2}{ }^{\mathrm{Mes}, \mathrm{Ph}} \mathrm{L}$ All hydrogen atoms apart from those bound to pyrrolyl $\mathrm{N}$ atoms have been omitted for clarity.

Table S2: Experimental metrical parameters (bond lengths in $\AA$ and angles in ${ }^{\circ}$ ) in $\mathrm{H}_{2}{ }^{\mathrm{Mes}, \mathrm{Ph}} \mathrm{L}$.

\begin{tabular}{cc}
\hline $\mathrm{C}(5)-\mathrm{N}(2)$ & $1.3534(17)$ \\
$\mathrm{C}(5)-\mathrm{C}(6)$ & $1.3995(18)$ \\
$\mathrm{C}(6)-\mathrm{C}(7)$ & $1.379(2)$ \\
$\mathrm{C}(7)-\mathrm{C}(8)$ & $1.382(2)$ \\
$\mathrm{C}(8)-\mathrm{C}(9)$ & $1.3958(19)$ \\
$\mathrm{C}(9)-\mathrm{N}(2)$ & $1.3528(17)$ \\
$\mathrm{C}(5 \mathrm{a})-\mathrm{N}(2 \mathrm{a})$ & $1.3528(17)$ \\
$\mathrm{C}(5 \mathrm{a})-\mathrm{C}(6 \mathrm{a})$ & $1.3979(18)$ \\
$\mathrm{C}(6 \mathrm{a})-\mathrm{C}(7 \mathrm{a})$ & $1.379(2)$ \\
$\mathrm{C}(7 \mathrm{a})-\mathrm{C}(8 \mathrm{a})$ & $1.380(2)$ \\
$\mathrm{C}(8 \mathrm{a})-\mathrm{C}(9 \mathrm{a})$ & $1.4018(18)$ \\
$\mathrm{C}(9 \mathrm{a})-\mathrm{N}(2 \mathrm{a})$ & $1.3517(17)$
\end{tabular}




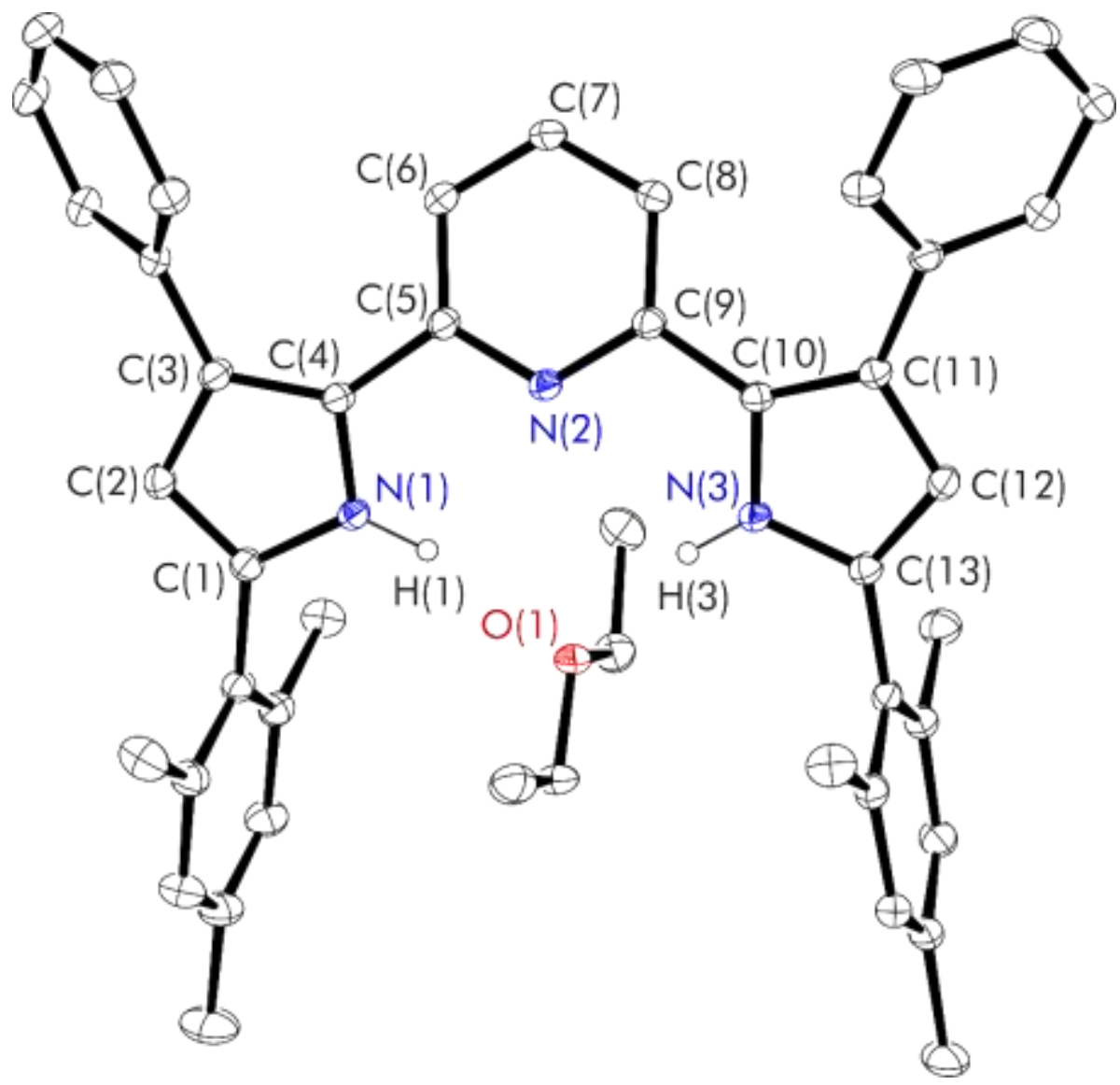

Figure S45: Thermal displacement ellipsoid drawing (50\% probability) of $\mathrm{H}_{2}{ }^{\mathrm{Mes}, \mathrm{Ph}} \mathrm{L} \cdot \mathrm{Et}_{2} \mathrm{O}$ All hydrogen atoms apart from those bound to pyrrolyl $\mathrm{N}$ atoms have been omitted for clarity.

Table S3: Experimental metrical parameters (bond lengths in $\AA$ and angles in ${ }^{\circ}$ ) in $\mathrm{H}_{2}{ }^{\mathrm{Mes}, \mathrm{Ph}^{2}} \mathrm{~L} \cdot \mathrm{Et}_{2} \mathrm{O}$.

\begin{tabular}{lc}
\hline $\mathrm{C}(5)-\mathrm{N}(2)$ & $1.3458(17)$ \\
$\mathrm{C}(5)-\mathrm{C}(6)$ & $1.3953(18)$ \\
$\mathrm{C}(6)-\mathrm{C}(7)$ & $1.3897(19)$ \\
$\mathrm{C}(7)-\mathrm{C}(8)$ & $1.386(2)$ \\
$\mathrm{C}(8)-\mathrm{C}(9)$ & $1.3987(18)$ \\
$\mathrm{C}(9)-\mathrm{N}(2)$ & $1.3493(17)$ \\
$\mathrm{O}(1)-\mathrm{H}(1)$ & 2.13 \\
$\mathrm{O}(1)-\mathrm{H}(3)$ & 2.12
\end{tabular}




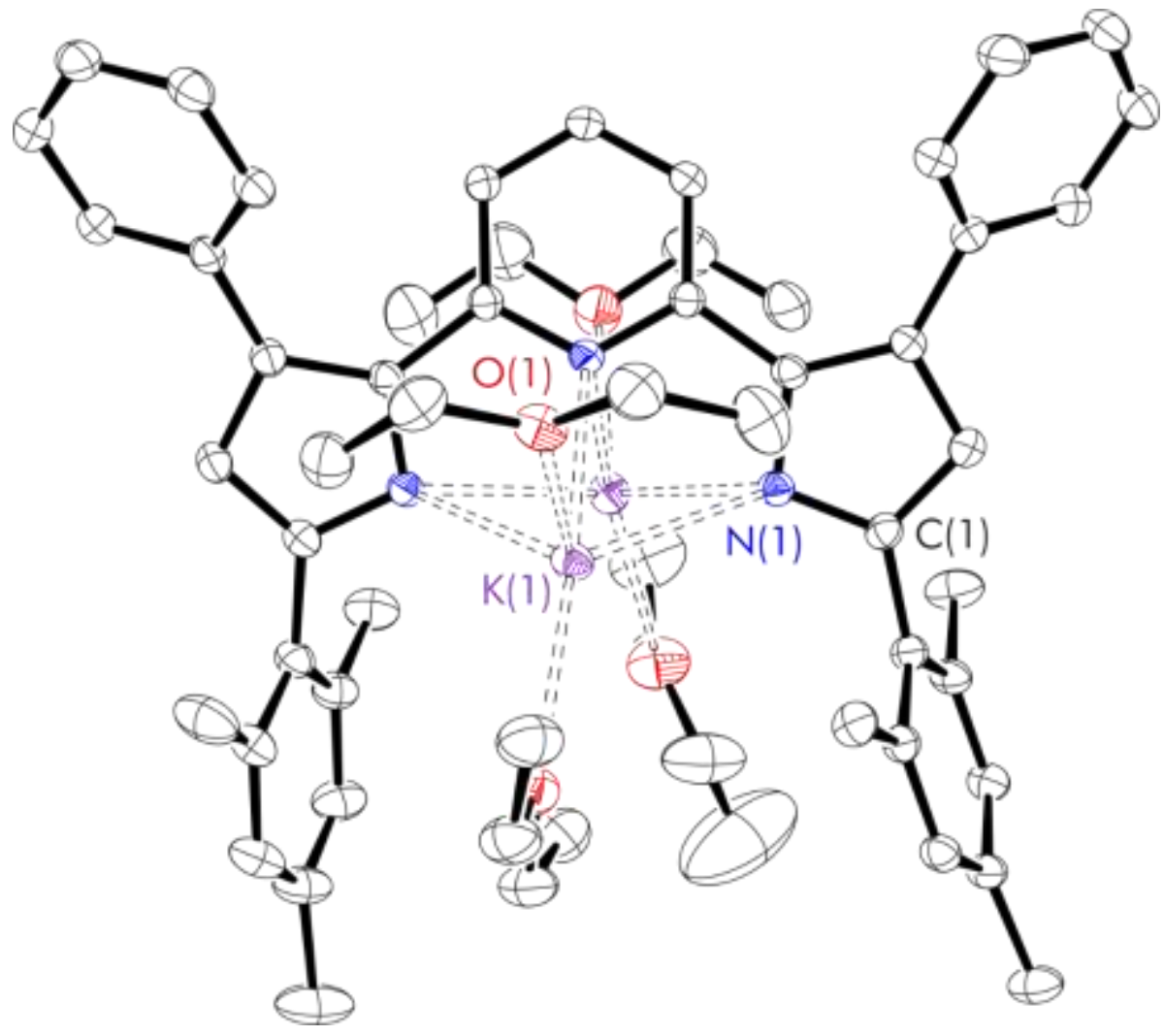

Figure S46: Thermal displacement ellipsoid drawings (30\% probability) of $\mathrm{K}_{2}{ }^{\mathrm{Mes}, \mathrm{Ph}} \mathrm{L}\left(\mathrm{Et} \mathrm{t}_{2} \mathrm{O}\right)_{4}$ $\left(\mathbf{1} \cdot\left(\mathbf{E t}_{2} \mathbf{O}\right)_{4}\right)$. All hydrogen atoms have been omitted for clarity.

Table S4: Experimental metrical parameters (bond lengths in $\AA$ and angles in ${ }^{\circ}$ ) in $\mathrm{K}_{2}{ }^{\mathrm{Mes}, \mathrm{Ph}} \mathrm{L}\left(\mathrm{Et}_{2} \mathrm{O}\right)_{4}$ $\left(\mathbf{1} \cdot\left(\mathbf{E t}_{2} \mathbf{O}\right)_{4}\right)$.

\begin{tabular}{cc}
\hline $\mathrm{K}(1)-\mathrm{K}(2)$ & $3.6478(10)$ \\
$\mathrm{K}(1)-\mathrm{O}(1)$ & $2.718(3)$ \\
$\mathrm{K}(1)-\mathrm{O}(2)$ & $2.772(3)$ \\
$\mathrm{K}(1)-\mathrm{N}(1)$ & $2.901(3)$ \\
$\mathrm{K}(1)-\mathrm{N}(2)$ & $2.692(3)$ \\
$\mathrm{K}(1)-\mathrm{N}(3)$ & $2.917(3)$ \\
$\mathrm{K}(2)-\mathrm{N}(1)$ & $2.851(3)$ \\
$\mathrm{K}(2)-\mathrm{N}(2)$ & $2.777(3)$ \\
$\mathrm{K}(2)-\mathrm{N}(3)$ & $2.872(3)$ \\
$\mathrm{C}(5)-\mathrm{N}(2)$ & $1.347(4)$ \\
$\mathrm{C}(5)-\mathrm{C}(6)$ & $1.401(4)$ \\
$\mathrm{C}(6)-\mathrm{C}(7)$ & $1.383(5)$ \\
$\mathrm{C}(7)-\mathrm{C}(8)$ & $1.385(5)$ \\
$\mathrm{C}(8)-\mathrm{C}(9)$ & $1.404(4)$ \\
$\mathrm{C}(9)-\mathrm{N}(2)$ & $1.348(4)$ \\
$\mathrm{K}(1)-\mathrm{N}(1)-\mathrm{K}(2)$ & $78.72(7)$ \\
$\mathrm{K}(1)-\mathrm{N}(2)-\mathrm{K}(2)$ & $83.66(7)$ \\
$\mathrm{N}(1)-\mathrm{K}(1)-\mathrm{N}(3)$ & $97.76(8)$
\end{tabular}




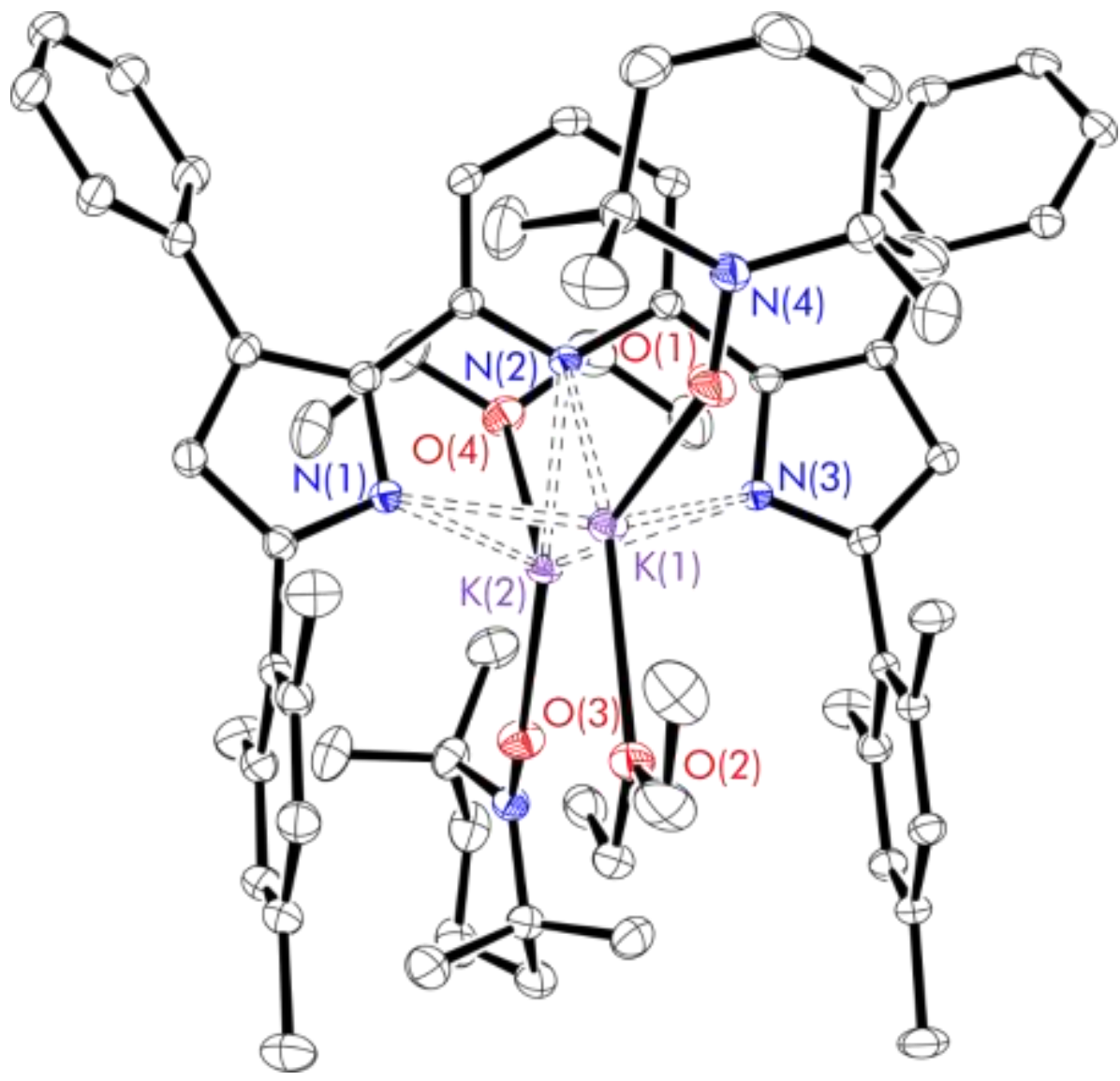

Figure S47: Thermal displacement ellipsoid drawings (30\% probability) of $\mathrm{K}_{2}{ }^{\mathrm{Mes}, \mathrm{Ph}} \mathrm{L}(\mathrm{OTEMP})_{2}\left(\mathrm{Et}_{2} \mathrm{O}\right)_{2}$ All hydrogen atoms have been omitted for clarity.

Table S5: Experimental metrical parameters (bond lengths in $\AA$ and angles in ${ }^{\circ}$ ) in $\mathrm{K}_{2}{ }^{\mathrm{Mes}, \mathrm{Ph}} \mathrm{L}(\mathrm{OTEMP})_{2}\left(\mathrm{Et}_{2} \mathrm{O}\right)_{2}$.

\begin{tabular}{cc}
\hline $\mathrm{K}(1)-\mathrm{K}(2)$ & $3.5313(4)$ \\
$\mathrm{K}(1)-\mathrm{O}(1)$ & $2.5627(12)$ \\
$\mathrm{K}(1)-\mathrm{O}(2)$ & $2.7836(11)$ \\
$\mathrm{K}(1)-\mathrm{N}(1)$ & $2.8095(11)$ \\
$\mathrm{K}(1)-\mathrm{N}(2)$ & $2.8543(11)$ \\
$\mathrm{K}(1)-\mathrm{N}(3)$ & $2.7846(12)$ \\
$\mathrm{K}(2)-\mathrm{N}(1)$ & $2.9303(12)$ \\
$\mathrm{K}(2)-\mathrm{N}(2)$ & $2.7520(11)$ \\
$\mathrm{K}(3)-\mathrm{N}(3)$ & $2.8854(11)$ \\
$\mathrm{C}(5)-\mathrm{N}(2)$ & $1.3464(17)$ \\
$\mathrm{C}(5)-\mathrm{C}(6)$ & $1.4058(18)$ \\
$\mathrm{C}(6)-\mathrm{C}(7)$ & $1.385(2)$ \\
$\mathrm{C}(7)-\mathrm{C}(8)$ & $1.383(2)$ \\
$\mathrm{C}(8)-\mathrm{C}(9)$ & $1.4027(18)$ \\
$\mathrm{C}(9)-\mathrm{N}(2)$ & $1.3519(17)$ \\
$\mathrm{K}(1)-\mathrm{N}(1)-\mathrm{K}(2)$ & $75.90(3)$ \\
$\mathrm{K}(1)-\mathrm{N}(2)-\mathrm{K}(2)$ & $78.06(3)$ \\
$\mathrm{N}(1)-\mathrm{K}(1)-\mathrm{N}(3)$ & $101.24(3)$
\end{tabular}




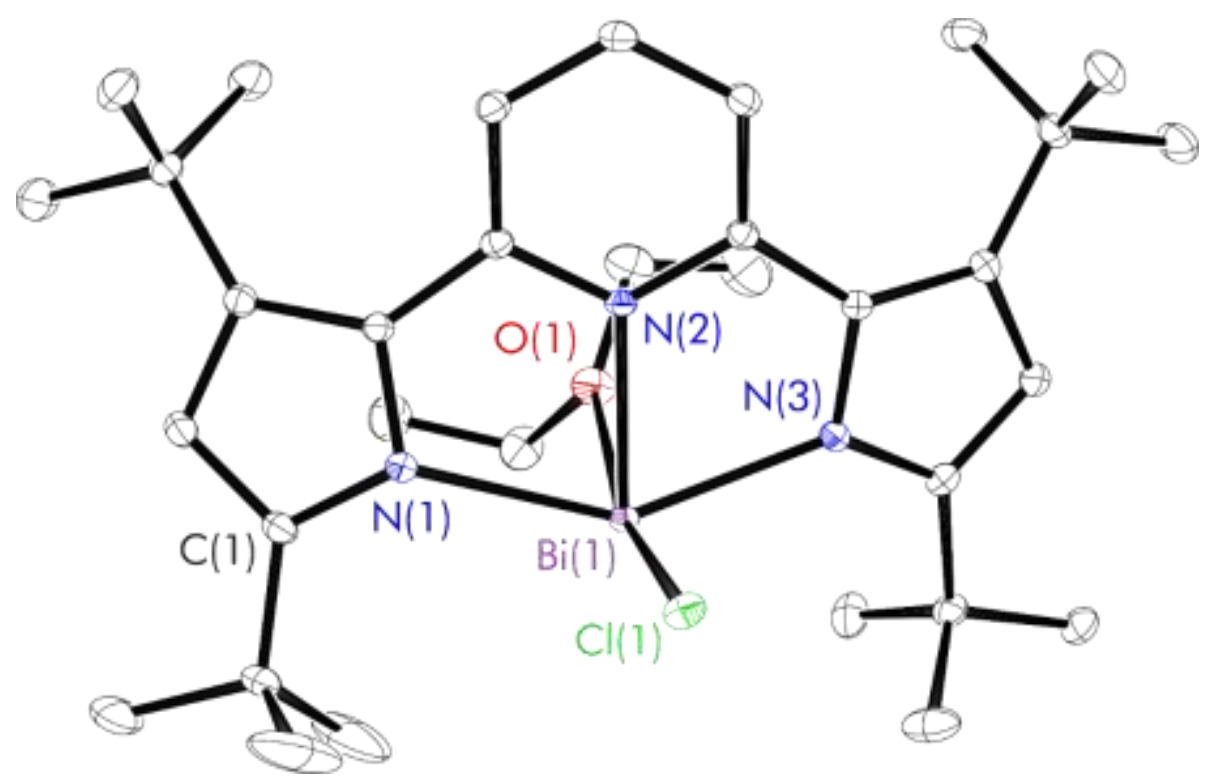

Figure S48: Thermal displacement ellipsoid drawing (30\% probability) of $\left({ }^{\mathrm{t} B u}{ }^{\mathrm{t} B \mathrm{Bu}} \mathrm{L}\right) \mathrm{BiCl}\left(\mathrm{Et}_{2} \mathrm{O}\right)$ $\left(\mathbf{3} \cdot \mathbf{E t}_{2} \mathbf{O}\right)$. All hydrogen atoms have been omitted and ${ }^{\mathrm{B} u}$ groups have been truncated for clarity.

Table S6: Experimental metrical parameters (bond lengths in $\AA$ and angles in ${ }^{\circ}$ ) in $\left({ }^{\mathrm{t}}{ }^{\mathrm{Bu}}{ }^{\mathrm{B}}{ }_{\mathrm{Bu}} \mathrm{L}\right) \mathrm{BiCl}\left(\mathrm{Et}_{2} \mathrm{O}\right)\left(\mathbf{3} \cdot \mathbf{E t}_{2} \mathbf{O}\right)$.

\begin{tabular}{cc}
\hline $\mathrm{Bi}(1)-\mathrm{Cl}(1)$ & $2.5199(4)$ \\
$\mathrm{Bi}(1)-\mathrm{O}(1)$ & $2.6401(15)$ \\
$\mathrm{Bi}(1)-\mathrm{N}(1)$ & $2.3366(15)$ \\
$\mathrm{Bi}(1)-\mathrm{N}(2)$ & $2.2339(15)$ \\
$\mathrm{Bi}(1)-\mathrm{N}(3)$ & $2.2918(15)$ \\
$\mathrm{C}(5)-\mathrm{N}(2)$ & $1.367(2)$ \\
$\mathrm{C}(5)-\mathrm{C}(6)$ & $1.396(3)$ \\
$\mathrm{C}(6)-\mathrm{C}(7)$ & $1.379(3)$ \\
$\mathrm{C}(7)-\mathrm{C}(8)$ & $1.378(3)$ \\
$\mathrm{C}(8)-\mathrm{C}(9)$ & $1.397(3)$ \\
$\mathrm{C}(9)-\mathrm{N}(2)$ & $1.372(2)$ \\
$\mathrm{N}(1)-\mathrm{Bi}(1)-\mathrm{N}(3)$ & $144.03(5)$ \\
$\mathrm{N}(1)-\mathrm{Bi}(1)-\mathrm{N}(2)$ & $72.20(6)$ \\
$\mathrm{N}(2)-\mathrm{Bi}(1)-\mathrm{N}(3)$ & $71.85(6)$ \\
$\mathrm{N}(1)-\mathrm{Bi}(1)-\mathrm{Cl}(1)$ & $91.41(4)$ \\
$\mathrm{N}(2)-\mathrm{Bi}(1)-\mathrm{Cl}(1)$ & $92.14(4)$ \\
$\mathrm{N}(3)-\mathrm{Bi}(1)-\mathrm{Cl}(1)$ & $88.63(4)$ \\
$\mathrm{O}(1)-\mathrm{Bi}(1)-\mathrm{Cl}(1)$ & $166.50(4)$
\end{tabular}




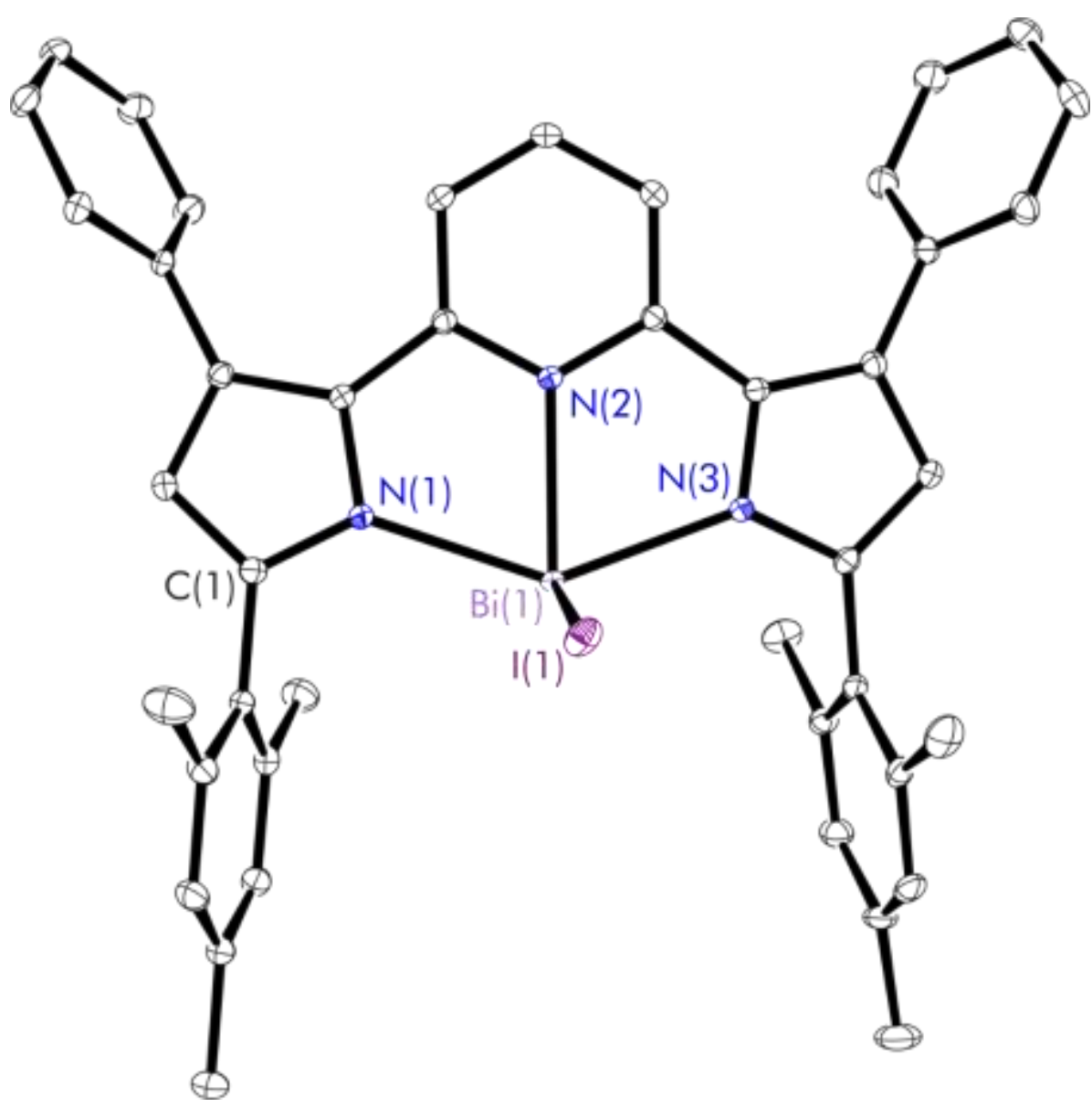

Figure S49: Thermal displacement ellipsoid drawing (30\% probability) of ( $\left.{ }^{\mathrm{Mes}, \mathrm{Ph}} \mathrm{L}\right) \mathrm{BiI}$ (4). All hydrogen atoms have been omitted for clarity. 


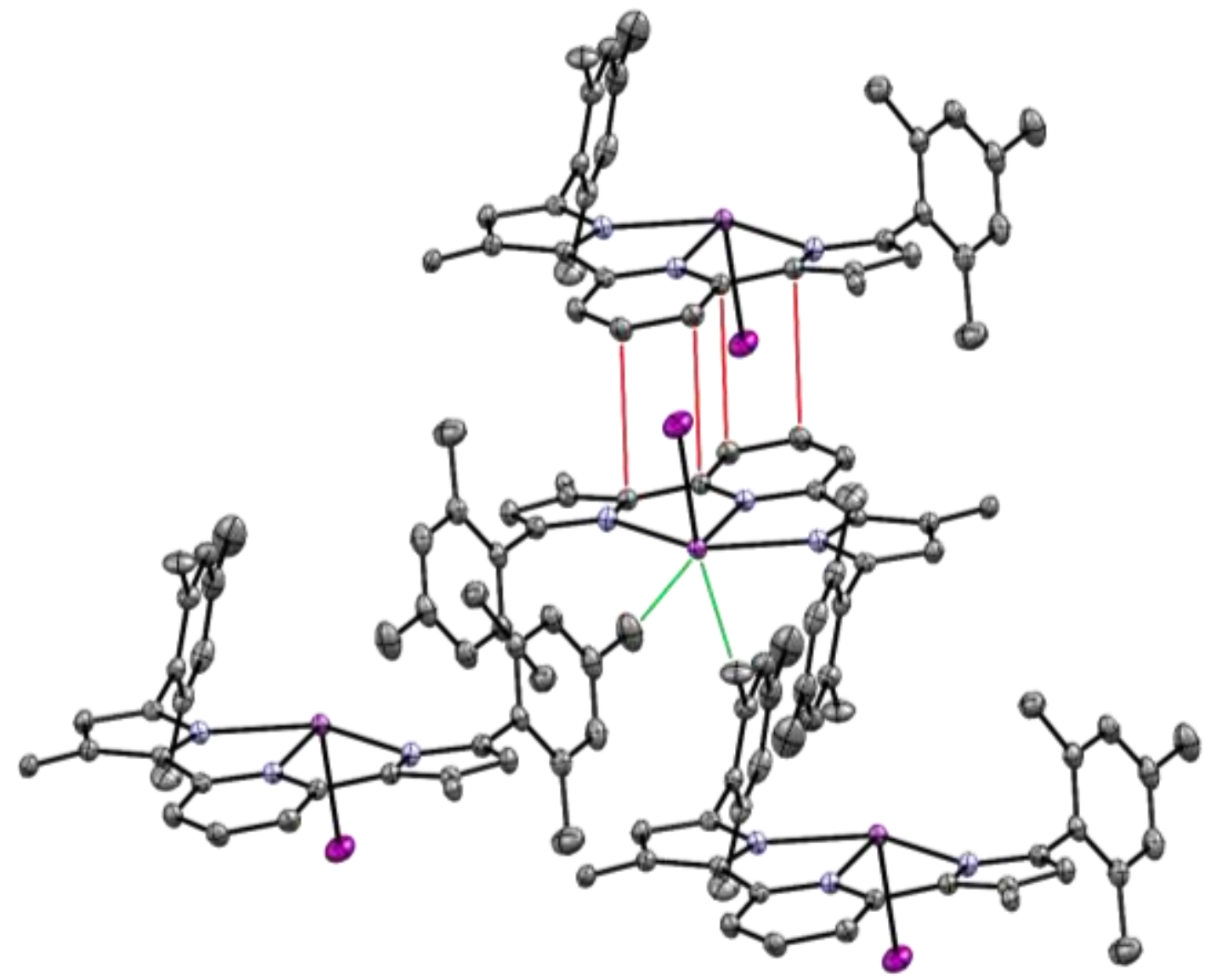

Figure S50: Representation of the extended structure of ( $\left.{ }^{\mathrm{Mes}, \mathrm{Ph}} \mathrm{L}\right) \mathrm{BiI}(4)$. On the central molecule, short $\mathrm{Bi} \cdot \mathrm{H}_{\mathrm{Mes}}$ interactions $\mathrm{cis}$ and trans to iodide highlighted by solid green lines and $\pi$-stacking highlighted by solid red lines. All hydrogen atoms have been omitted and phenyl groups have been truncated for clarity.

Table S7: Experimental metrical parameters (bond lengths in $\AA$ and angles in ${ }^{\circ}$ ) in ( $\left.{ }^{\mathrm{Mes}, \mathrm{Ph}} \mathrm{L}\right) \mathrm{BiI}(\mathbf{4})$.

\begin{tabular}{cc}
\hline $\mathrm{Bi}(1)-\mathrm{I}(1)$ & $2.8128(2)$ \\
$\mathrm{Bi}(1)-\mathrm{N}(1)$ & $2.3018(19)$ \\
$\mathrm{Bi}(1)-\mathrm{N}(2)$ & $2.3017(19)$ \\
$\mathrm{Bi}(1)-\mathrm{N}(3)$ & $2.3039(19)$ \\
$\mathrm{C}(5)-\mathrm{N}(2)$ & $1.362(3)$ \\
$\mathrm{C}(5)-\mathrm{C}(6)$ & $1.397(3)$ \\
$\mathrm{C}(6)-\mathrm{C}(7)$ & $1.383(3)$ \\
$\mathrm{C}(7)-\mathrm{C}(8)$ & $1.386(3)$ \\
$\mathrm{C}(8)-\mathrm{C}(9)$ & $1.395(3)$ \\
$\mathrm{C}(9)-\mathrm{N}(2)$ & $1.365(3)$ \\
$\mathrm{N}(1)-\mathrm{Bi}(1)-\mathrm{N}(3)$ & $140.78(7)$ \\
$\mathrm{N}(1)-\mathrm{Bi}(1)-\mathrm{N}(2)$ & $70.67(7)$ \\
$\mathrm{N}(2)-\mathrm{Bi}(1)-\mathrm{N}(3)$ & $70.20(7)$ \\
$\mathrm{N}(1)-\mathrm{Bi}(1)-\mathrm{I}(1)$ & $92.55(5)$ \\
$\mathrm{N}(2)-\mathrm{Bi}(1)-\mathrm{I}(1)$ & $99.93(5)$ \\
$\mathrm{N}(3)-\mathrm{Bi}(1)-\mathrm{I}(1)$ & $91.65(5)$
\end{tabular}




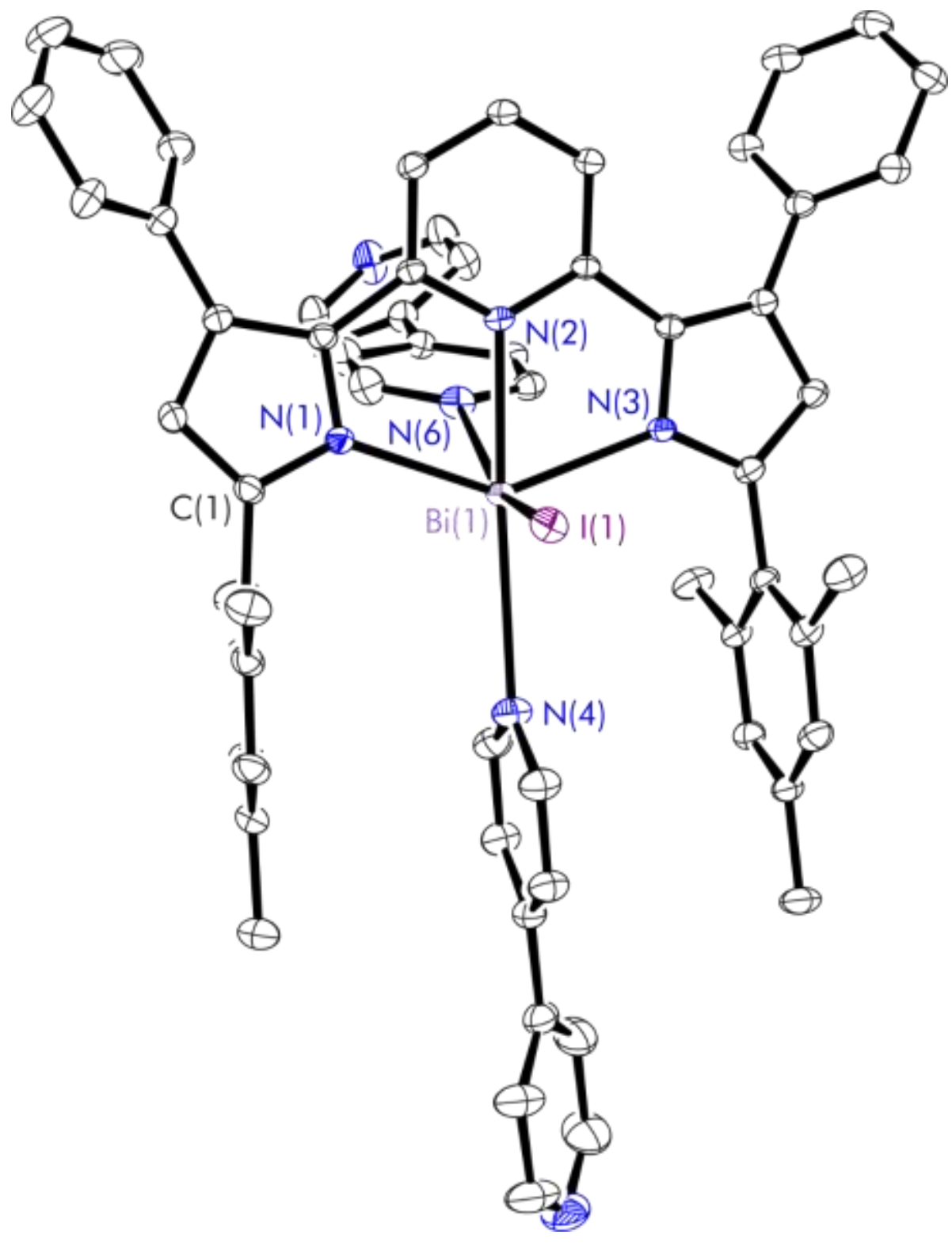

Figure S51: Thermal displacement ellipsoid drawing (30\% probability) of ( $\left.{ }^{\mathrm{Mes}, \mathrm{Ph}} \mathrm{L}\right) \mathrm{BiI}(4,4 \text { '-bipy })_{2}$ $(\mathbf{4} \cdot \mathbf{4 , 4}$ '-bipy). All hydrogen atoms have been omitted for clarity. 


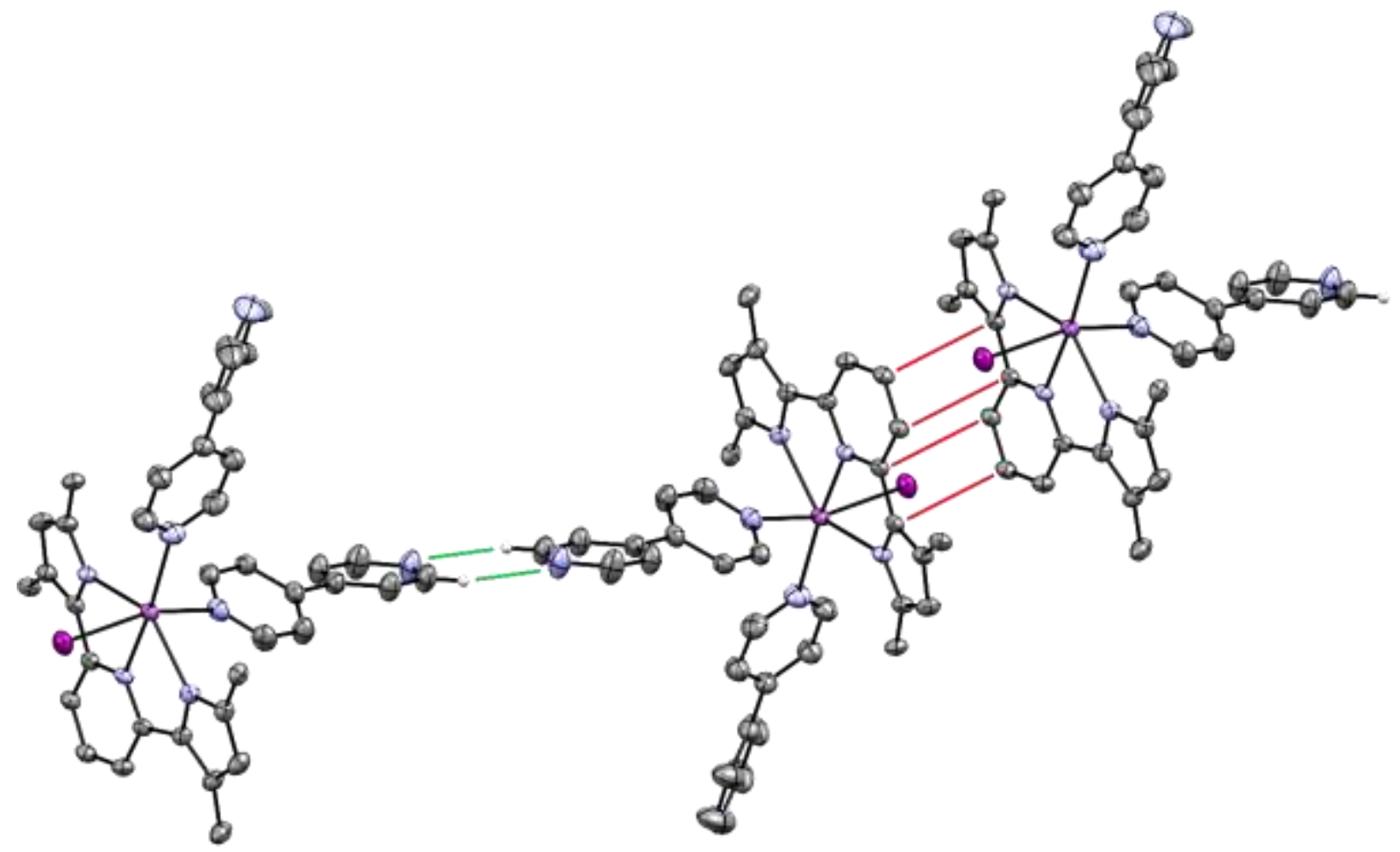

Figure S52: Representation of the extended structure of $\left({ }^{\mathrm{Mes}, \mathrm{Ph}} \mathrm{L}\right) \mathrm{BiI}(4,4 \text { '-bipy })_{2}$ Intermolecular $\mathrm{N}_{\text {bipy }} \cdot \mathrm{H}_{\text {bipy }}$ interactions highlighted by green solid lines and $\pi$-stacking highlighted by red solid lines. All other hydrogen atoms have been omitted and phenyl/mesityl groups have been truncated for clarity.

Table S8: Experimental metrical parameters (bond lengths in $\AA$ and angles in ${ }^{\circ}$ ) in $\left({ }^{\text {Mes,Ph }} \mathrm{L}\right) \mathrm{BiI}(4,4 \text { '-bipy })_{2}(\mathbf{4} \cdot \mathbf{4 , 4}$ '-bipy $)$.

\begin{tabular}{cc}
\hline $\mathrm{Bi}(1)-\mathrm{I}(1)$ & $2.8824(2)$ \\
$\mathrm{Bi}(1)-\mathrm{N}(1)$ & $2.3204(19)$ \\
$\mathrm{Bi}(1)-\mathrm{N}(2)$ & $2.3257(19)$ \\
$\mathrm{Bi}(1)-\mathrm{N}(3)$ & $2.3343(19)$ \\
$\mathrm{Bi}(1)-\mathrm{N}(4)$ & $2.9096(1)$ \\
$\mathrm{Bi}(1)-\mathrm{N}(6)$ & $2.769(2)$ \\
$\mathrm{C}(5)-\mathrm{N}(2)$ & $1.365(3)$ \\
$\mathrm{C}(5)-\mathrm{C}(6)$ & $1.399(3)$ \\
$\mathrm{C}(6)-\mathrm{C}(7)$ & $1.387(4)$ \\
$\mathrm{C}(7)-\mathrm{C}(8)$ & $1.381(3)$ \\
$\mathrm{C}(8)-\mathrm{C}(9)$ & $1.404(3)$ \\
$\mathrm{C}(9)-\mathrm{N}(2)$ & $1.359(3)$ \\
$\mathrm{N}(1)-\mathrm{Bi}(1)-\mathrm{N}(3)$ & $139.89(7)$ \\
$\mathrm{N}(1)-\mathrm{Bi}(1)-\mathrm{N}(2)$ & $69.96(7)$ \\
$\mathrm{N}(2)-\mathrm{Bi}(1)-\mathrm{N}(3)$ & $70.08(7)$ \\
$\mathrm{N}(1)-\mathrm{Bi}(1)-\mathrm{I}(1)$ & $87.61(5)$ \\
$\mathrm{N}(2)-\mathrm{Bi}(1)-\mathrm{I}(1)$ & $91.26(5)$ \\
$\mathrm{N}(3)-\mathrm{Bi}(1)-\mathrm{I}(1)$ & $89.93(5)$ \\
$\mathrm{N}(6)-\mathrm{Bi}(1)-\mathrm{I}(1)$ & $160.90(5)$
\end{tabular}




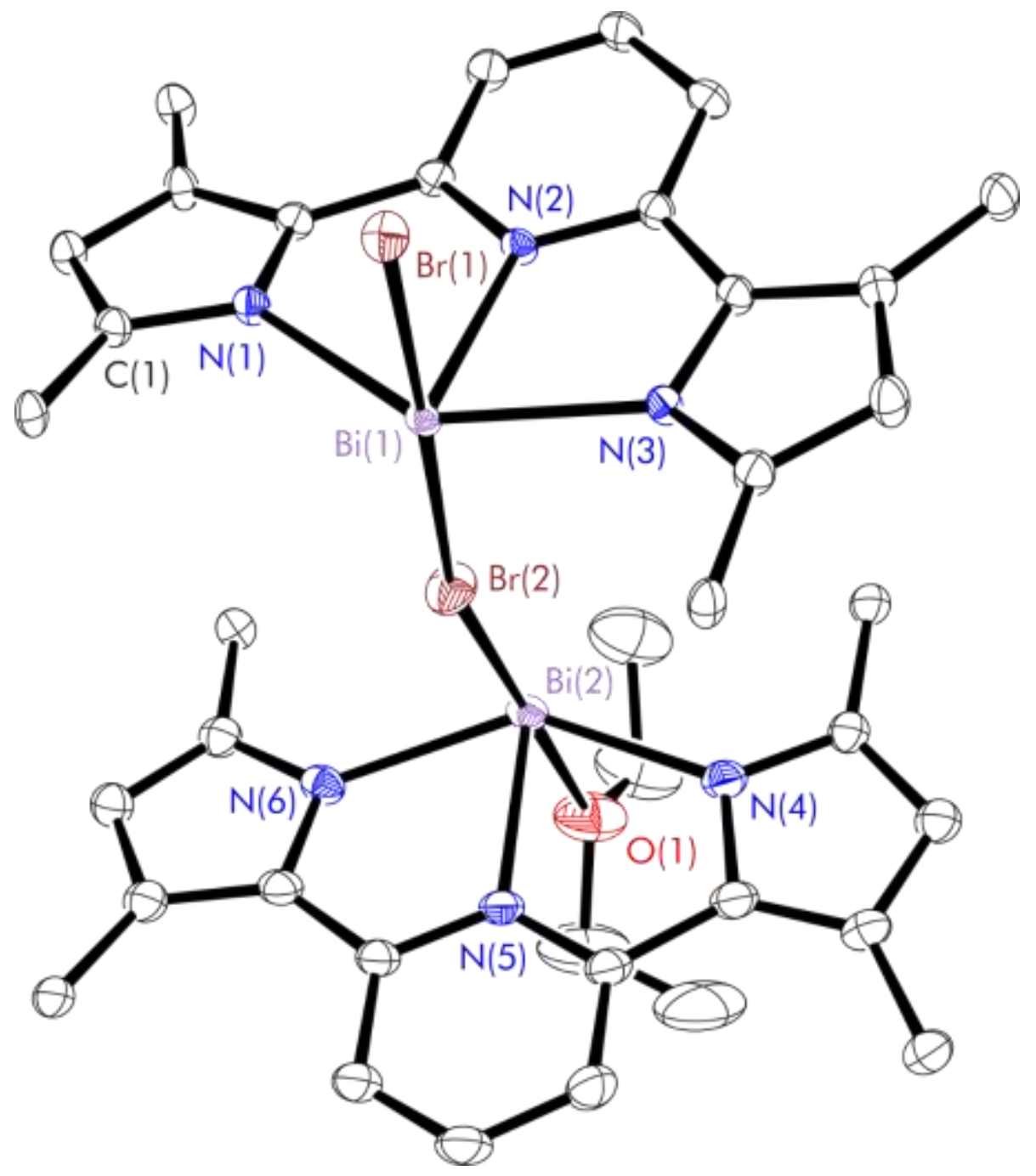

Figure S53: Thermal displacement ellipsoid drawing (30\% probability) of $\left\{\left({ }^{\mathrm{Mes}, \mathrm{Ph}} \mathrm{L}\right) \mathrm{Bi}\right\}_{2}(\mu-\mathrm{Br}) \mathrm{Br}\left(\mathrm{Et}_{2} \mathrm{O}\right)\left(\mathbf{5}_{\mathbf{2}} \mathbf{E t}_{\mathbf{2}} \mathbf{O}\right)$. All hydrogen atoms have been omitted and phenyl/mesityl groups have been truncated for clarity.

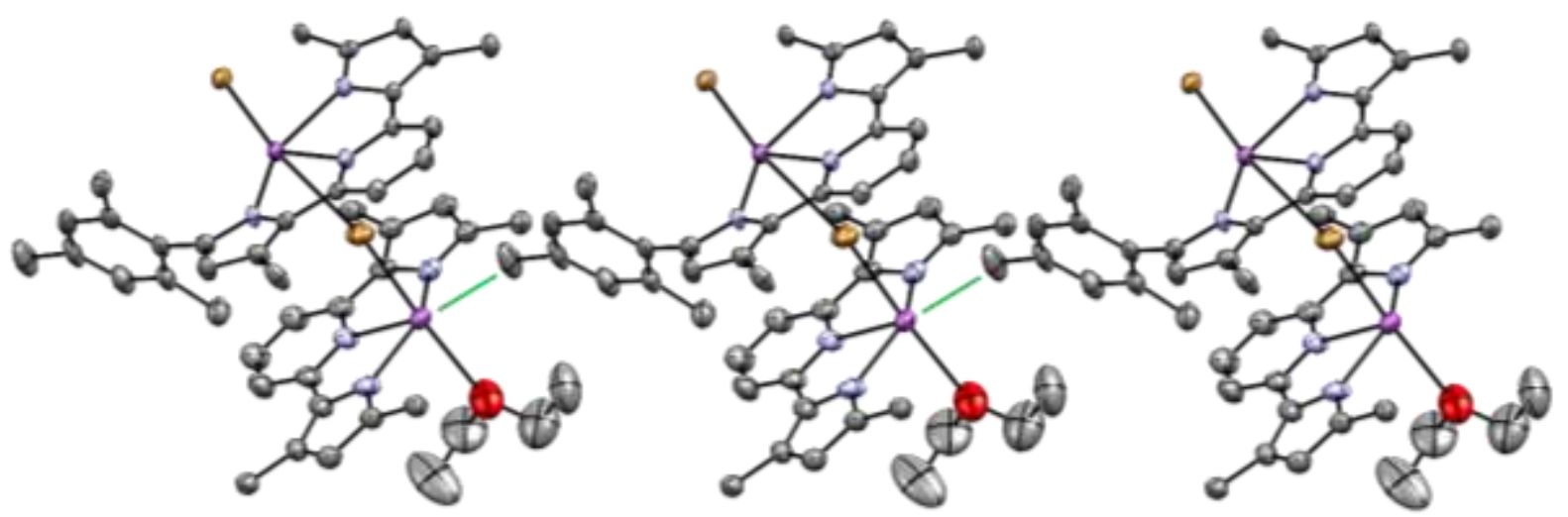

Figure S54: Representation of the extended structure of $\left\{\left({ }^{\mathrm{Mes}, \mathrm{Ph}} \mathrm{L}\right) \mathrm{Bi}\right\}_{2}(\mu-\mathrm{Br}) \mathrm{Br}\left(\mathrm{Et}_{2} \mathrm{O}\right)\left(\mathbf{5}_{\mathbf{2}} \mathbf{E t}_{\mathbf{2}} \mathbf{O}\right)$. Short $\mathrm{Bi} \cdot \cdot \mathrm{H}_{\mathrm{Mes}}$ interaction cis to $\mathrm{Br}(2)$ highlighted by solid green line. All hydrogen atoms have been omitted and phenyl/mesityl groups have been truncated where necessary for clarity. 
Table S9: Experimental metrical parameters (bond lengths in $\AA$ and angles in ${ }^{\circ}$ ) in $\left\{\left({ }^{\mathrm{Mes}, \mathrm{Ph}} \mathrm{L}\right) \mathrm{Bi}\right\}_{2}(\mu-\mathrm{Br}) \mathrm{Br}\left(\mathrm{Et}_{2} \mathrm{O}\right)\left(\mathbf{5}_{\mathbf{2}} \mathbf{E t}_{\mathbf{2}} \mathbf{O}\right)$.

\begin{tabular}{cc}
\hline $\mathrm{Bi}(1)-\mathrm{Br}(1)$ & $2.6363(5)$ \\
$\mathrm{Bi}(1)-\mathrm{Br}(2)$ & $3.3236(1)$ \\
$\mathrm{Bi}(2)-\mathrm{Br}(2)$ & $2.6976(6)$ \\
$\mathrm{Bi}(1)-\mathrm{N}(1)$ & $2.294(3)$ \\
$\mathrm{Bi}(1)-\mathrm{N}(2)$ & $2.301(3)$ \\
$\mathrm{Bi}(1)-\mathrm{N}(3)$ & $2.281(3)$ \\
$\mathrm{Bi}(2)-\mathrm{O}(1)$ & $2.705(6)$ \\
$\mathrm{Bi}(2)-\mathrm{N}(4)$ & $2.294(5)$ \\
$\mathrm{Bi}(2)-\mathrm{N}(5)$ & $2.297(4)$ \\
$\mathrm{Bi}(2)-\mathrm{N}(6)$ & $2.300(4)$ \\
$\mathrm{C}(5)-\mathrm{N}(2)$ & $1.366(5)$ \\
$\mathrm{C}(5)-\mathrm{C}(6)$ & $1.400(6)$ \\
$\mathrm{C}(6)-\mathrm{C}(7)$ & $1.381(6)$ \\
$\mathrm{C}(7)-\mathrm{C}(8)$ & $1.395(6)$ \\
$\mathrm{C}(8)-\mathrm{C}(9)$ & $1.400(6)$ \\
$\mathrm{C}(9)-\mathrm{N}(2)$ & $1.366(5)$ \\
$\mathrm{Bi}(1)-\mathrm{Br}(1)-\mathrm{Bi}(2)$ & $171.89(1)$ \\
$\mathrm{Br}(2)-\mathrm{Bi}(2)-\mathrm{O}(1)$ & $176.87(13)$ \\
$\mathrm{N}(1)-\mathrm{Bi}(1)-\mathrm{N}(3)$ & $139.92(12)$ \\
$\mathrm{N}(1)-\mathrm{Bi}(1)-\mathrm{N}(2)$ & $70.11(12)$ \\
$\mathrm{N}(2)-\mathrm{Bi}(1)-\mathrm{N}(3)$ & $69.97(12)$ \\
$\mathrm{N}(1)-\mathrm{Bi}(1)-\mathrm{Br}(1)$ & $90.56(9)$ \\
$\mathrm{N}(2)-\mathrm{Bi}(1)-\mathrm{Br}(1)$ & $92.33(9)$ \\
$\mathrm{N}(3)-\mathrm{Bi}(1)-\mathrm{Br}(1)$ & $87.62(9)$ \\
$\mathrm{N}(4)-\mathrm{Bi}(2)-\mathrm{N}(6)$ & $140.50(15)$ \\
$\mathrm{N}(4)-\mathrm{Bi}(1)-\mathrm{N}(5)$ & $70.32(15)$ \\
$\mathrm{N}(5)-\mathrm{Bi}(1)-\mathrm{N}(6)$ & $70.61(16)$ \\
&
\end{tabular}




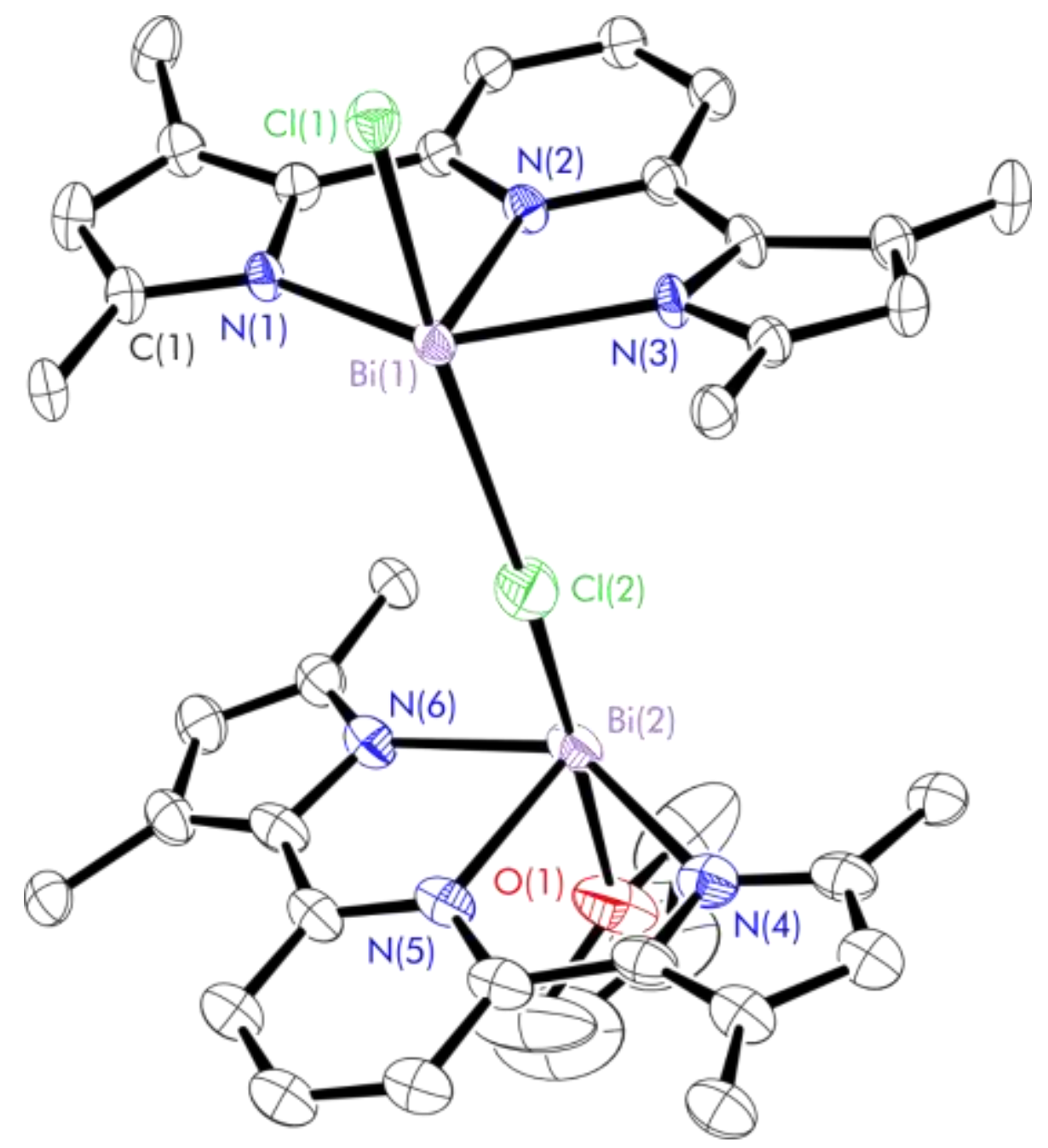

Figure S55: Thermal displacement ellipsoid drawing (30\% probability) of $\left\{\left({ }^{\mathrm{Mes}, \mathrm{Ph}} \mathrm{L}\right) \mathrm{Bi}\right\}_{2}(\mu-\mathrm{Cl}) \mathrm{Cl}(\mathrm{thf})$ $\left(\mathbf{6}_{2}\right.$ thf). All hydrogen atoms have been omitted and phenyl/mesityl groups have been truncated for clarity.

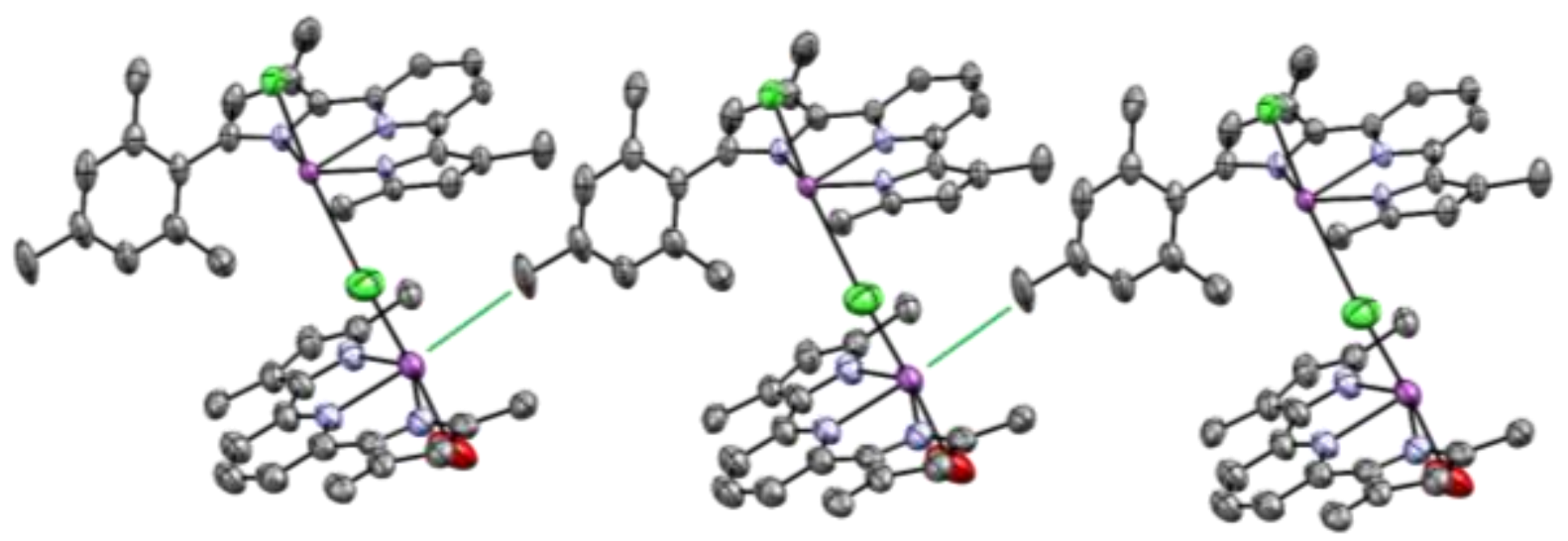

Figure S56: Representation of the extended structure of $\left\{\left({ }^{\mathrm{Mes}, \mathrm{Ph}} \mathrm{L}\right) \mathrm{Bi}\right\}_{2}(\mu-\mathrm{Cl}) \mathrm{Cl}(\mathrm{thf})\left(\mathbf{6}_{\mathbf{2}}\right.$ thf $)$. Short $\mathrm{Bi} \cdot \cdot \mathrm{H}_{\mathrm{Mes}}$ interaction cis to $\mathrm{Cl}(2)$ highlighted by solid green line. All hydrogen atoms have been omitted and phenyl/mesityl groups have been truncated where necessary for clarity. 
Table S10: Experimental metrical parameters (bond lengths in $\AA$ and angles in ${ }^{\circ}$ ) in $\left\{\left({ }^{\mathrm{Mes}, \mathrm{Ph}} \mathrm{L}\right) \mathrm{Bi}\right\}_{2}(\mu-\mathrm{Cl}) \mathrm{Cl}($ thf $)\left(\mathbf{6}_{\mathbf{2}}\right.$ thf $)$.

\begin{tabular}{cc}
\hline $\mathrm{Bi}(1)-\mathrm{Cl}(1)$ & $2.494(2)$ \\
$\mathrm{Bi}(1)-\mathrm{Cl}(2)$ & $3.1729(1)$ \\
$\mathrm{Bi}(2)-\mathrm{Cl}(2)$ & $2.584(3)$ \\
$\mathrm{Bi}(1)-\mathrm{N}(1)$ & $2.274(6)$ \\
$\mathrm{Bi}(1)-\mathrm{N}(2)$ & $2.302(5)$ \\
$\mathrm{Bi}(1)-\mathrm{N}(3)$ & $2.279(5)$ \\
$\mathrm{Bi}(2)-\mathrm{O}(1)$ & $2.553(10)$ \\
$\mathrm{Bi}(2)-\mathrm{N}(4)$ & $2.295(8)$ \\
$\mathrm{Bi}(2)-\mathrm{N}(5)$ & $2.279(7)$ \\
$\mathrm{Bi}(2)-\mathrm{N}(6)$ & $2.301(7)$ \\
$\mathrm{C}(5)-\mathrm{N}(2)$ & $1.364(9)$ \\
$\mathrm{C}(5)-\mathrm{C}(6)$ & $1.411(9)$ \\
$\mathrm{C}(6)-\mathrm{C}(7)$ & $1.371(10)$ \\
$\mathrm{C}(7)-\mathrm{C}(8)$ & $1.387(11)$ \\
$\mathrm{C}(8)-\mathrm{C}(9)$ & $1.392(10)$ \\
$\mathrm{C}(9)-\mathrm{N}(2)$ & $1.373(9)$ \\
$\mathrm{Cl}(1)-\mathrm{Bi}(1)-\mathrm{Cl}(2)$ & $175.38(1)$ \\
$\mathrm{Cl}(2)-\mathrm{Bi}(2)-\mathrm{O}(1)$ & $175.9(2)$ \\
$\mathrm{N}(1)-\mathrm{Bi}(1)-\mathrm{N}(3)$ & $139.7(2)$ \\
$\mathrm{N}(1)-\mathrm{Bi}(1)-\mathrm{N}(2)$ & $70.0(2)$ \\
$\mathrm{N}(2)-\mathrm{Bi}(1)-\mathrm{N}(3)$ & $70.0(2)$ \\
$\mathrm{N}(1)-\mathrm{Bi}(1)-\mathrm{Cl}(1)$ & $88.09(16)$ \\
$\mathrm{N}(2)-\mathrm{Bi}(1)-\mathrm{Cl}(1)$ & $88.91(16)$ \\
$\mathrm{N}(3)-\mathrm{Bi}(1)-\mathrm{Cl}(1)$ & $92.19(16)$ \\
$\mathrm{N}(4)-\mathrm{Bi}(2)-\mathrm{N}(6)$ & $139.9(3)$ \\
$\mathrm{N}(4)-\mathrm{Bi}(1)-\mathrm{N}(5)$ & $70.6(3)$ \\
$\mathrm{N}(5)-\mathrm{Bi}(1)-\mathrm{N}(6)$ & $70.4(3)$ \\
\end{tabular}




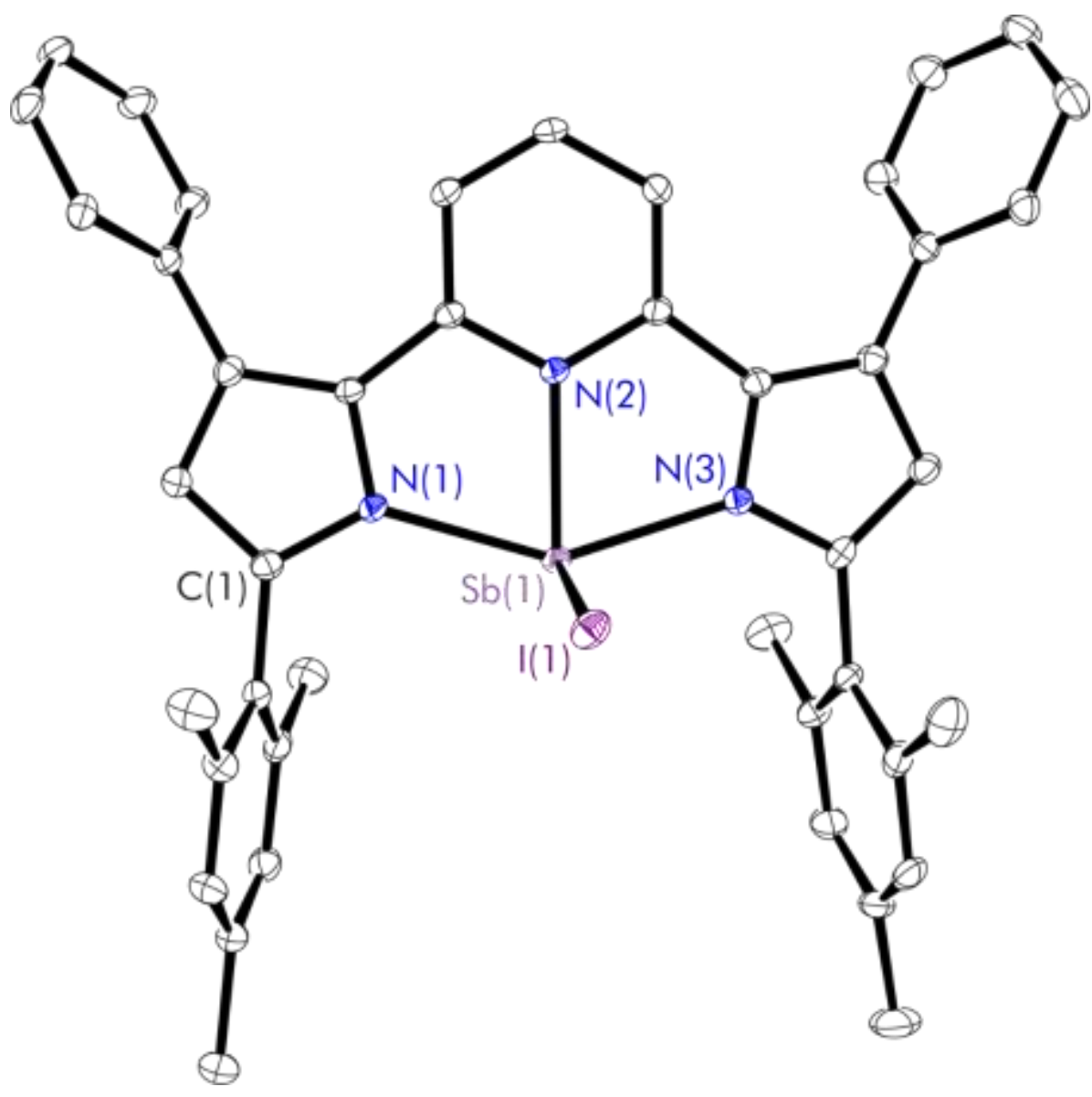

Figure S57: Thermal displacement ellipsoid drawings (30\% probability) of ( $\left.{ }^{\mathrm{Mes}, \mathrm{Ph}} \mathrm{L}\right) \mathrm{SbI}$ (7). All hydrogen atoms have been omitted for clarity. 


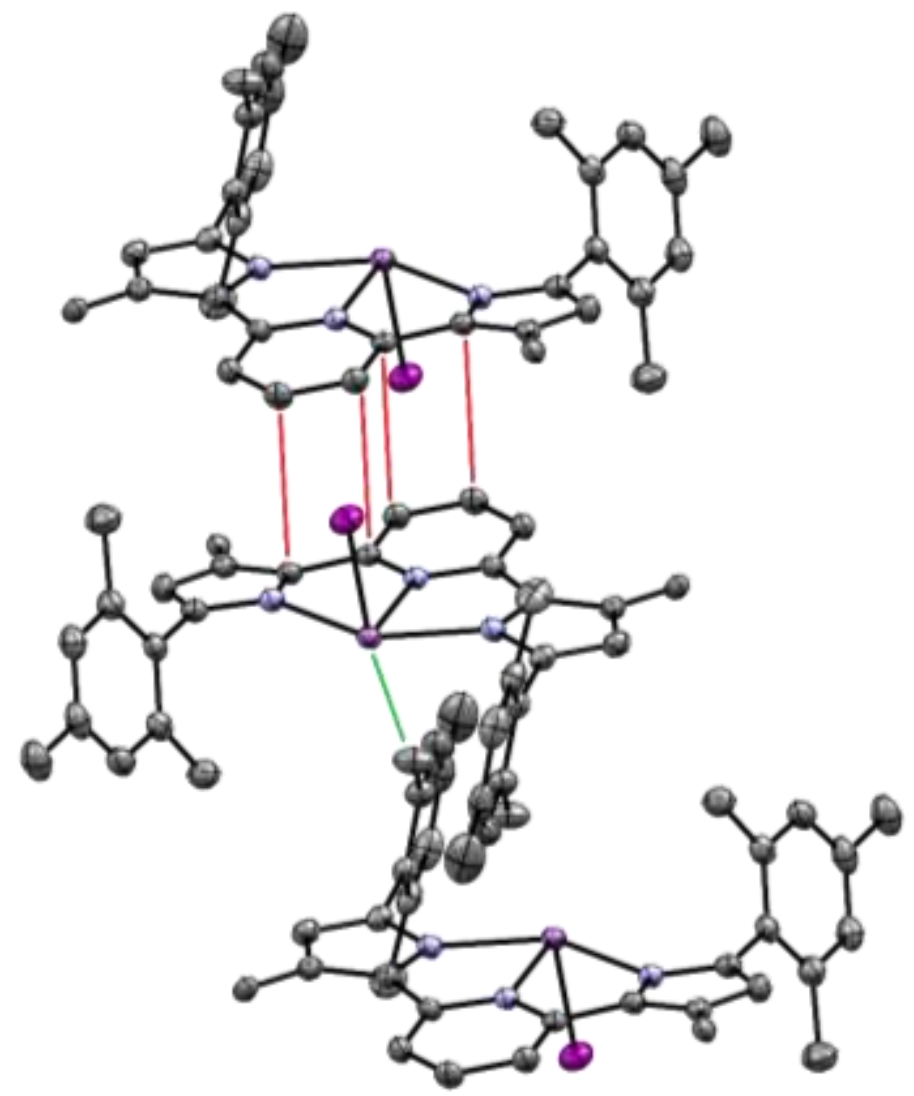

Figure S58: Representation of the extended structure of ( $\left.{ }^{\mathrm{Mes}, P h} \mathrm{~L}\right) \mathrm{SbI}(7)$. On the central molecule, short $\mathrm{Bi} \cdot \cdot \mathrm{H}_{\mathrm{Mes}}$ interaction trans to iodide highlighted by solid green line and $\pi$-stacking highlighted by solid red lines. All hydrogen atoms have been omitted and phenyl groups have been truncated for clarity.

Table S11: Experimental metrical parameters (bond lengths in $\AA$ and angles in ${ }^{\circ}$ ) in $\left({ }^{\mathrm{Mes}, \mathrm{Ph}} \mathrm{L}\right) \mathrm{SbI}(7)$.

\begin{tabular}{cc}
\hline $\mathrm{Sb}(1)-\mathrm{I}(1)$ & $2.7413(3)$ \\
$\mathrm{Sb}(1)-\mathrm{N}(1)$ & $2.216(2)$ \\
$\mathrm{Sb}(1)-\mathrm{N}(2)$ & $2.177(2)$ \\
$\mathrm{Sb}(1)-\mathrm{N}(3)$ & $2.207(3)$ \\
$\mathrm{C}(5)-\mathrm{N}(2)$ & $1.370(4)$ \\
$\mathrm{C}(5)-\mathrm{C}(6)$ & $1.398(4)$ \\
$\mathrm{C}(6)-\mathrm{C}(7)$ & $1.380(4)$ \\
$\mathrm{C}(7)-\mathrm{C}(8)$ & $1.393(4)$ \\
$\mathrm{C}(8)-\mathrm{C}(9)$ & $1.390(4)$ \\
$\mathrm{C}(9)-\mathrm{N}(2)$ & $1.369(4)$ \\
$\mathrm{N}(1)-\mathrm{Sb}(1)-\mathrm{N}(3)$ & $145.45(9)$ \\
$\mathrm{N}(1)-\mathrm{Sb}(1)-\mathrm{N}(2)$ & $73.07(9)$ \\
$\mathrm{N}(2)-\mathrm{Sb}(1)-\mathrm{N}(3)$ & $72.81(9)$ \\
$\mathrm{N}(1)-\mathrm{Sb}(1)-\mathrm{I}(1)$ & $91.14(6)$ \\
$\mathrm{N}(2)-\mathrm{Sb}(1)-\mathrm{I}(1)$ & $101.11(6)$ \\
$\mathrm{N}(3)-\mathrm{Sb}(1)-\mathrm{I}(1)$ & $90.17(7)$
\end{tabular}




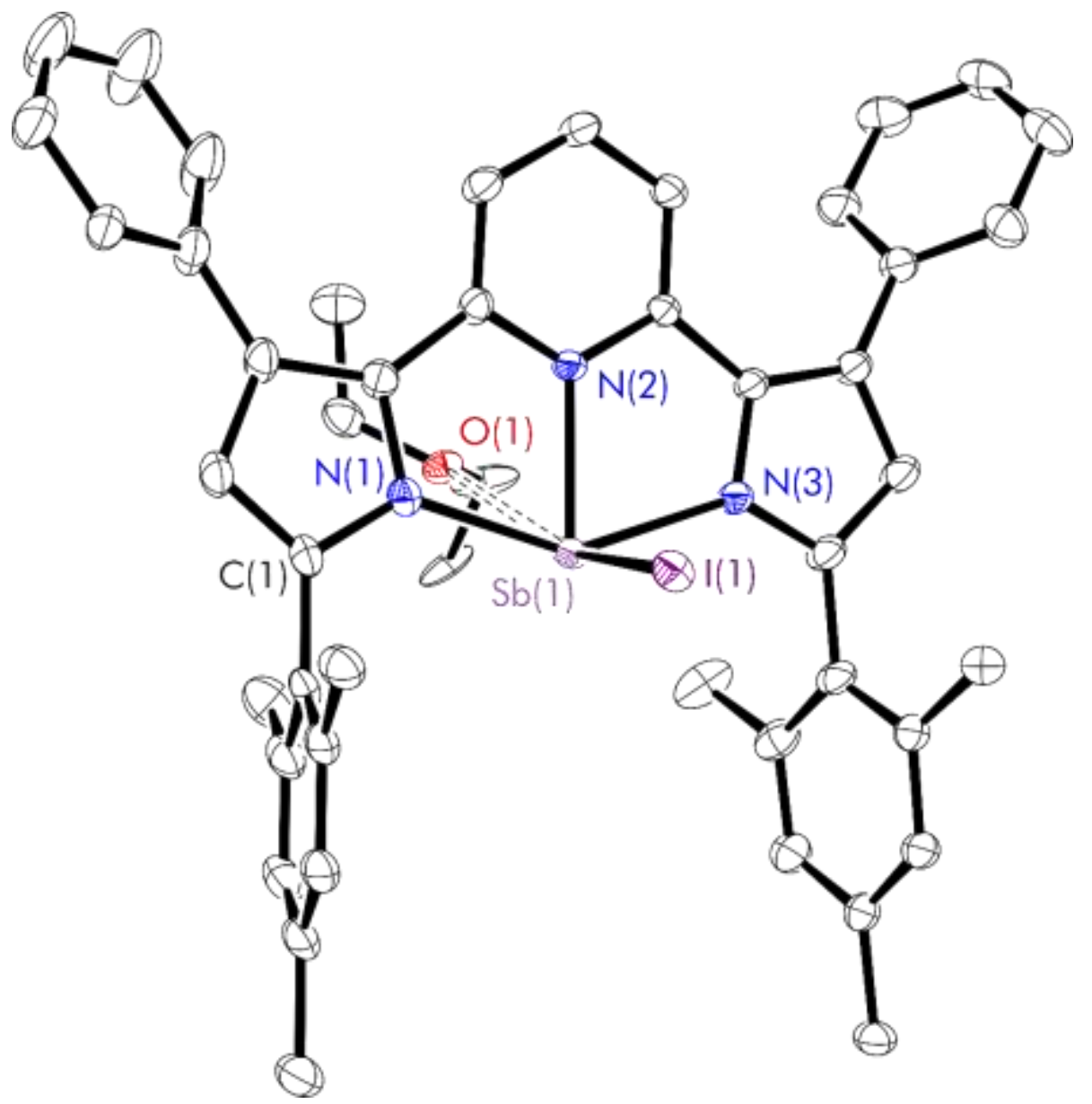

Figure S59: Thermal displacement ellipsoid drawings (30\% probability) of $\left({ }^{\mathrm{Mes}, \mathrm{Ph}} \mathrm{L}\right) \mathrm{SbI}\left(\mathrm{Et}_{2} \mathrm{O}\right)\left(\mathbf{7} \cdot \mathbf{E t}_{2} \mathbf{O}\right)$ All hydrogen atoms have been omitted and only one molecule depicted for clarity.

Table S12: Experimental metrical parameters (bond lengths in $\AA$ and angles in ${ }^{\circ}$ ) in $\left({ }^{\mathrm{Mes}, \mathrm{Ph}} \mathrm{L}\right) \mathrm{SbI}\left(\mathrm{Et}_{2} \mathrm{O}\right)\left(\mathbf{7} \cdot \mathbf{E t}_{\mathbf{2}} \mathbf{O}\right)$. Values correspond to the molecule depicted in Figure S59.

\begin{tabular}{cc}
\hline $\mathrm{Sb}(1)-\mathrm{I}(1)$ & $2.7742(5)$ \\
$\mathrm{Sb}(1)-\mathrm{N}(1)$ & $2.217(4)$ \\
$\mathrm{Sb}(1)-\mathrm{N}(2)$ & $2.179(4)$ \\
$\mathrm{Sb}(1)-\mathrm{N}(3)$ & $2.208(4)$ \\
$\mathrm{Sb}(1)-\mathrm{O}(1)$ & $2.9146(1)$ \\
$\mathrm{C}(5)-\mathrm{N}(2)$ & $1.355(7)$ \\
$\mathrm{C}(5)-\mathrm{C}(6)$ & $1.403(7)$ \\
$\mathrm{C}(6)-\mathrm{C}(7)$ & $1.379(7)$ \\
$\mathrm{C}(7)-\mathrm{C}(8)$ & $1.383(8)$ \\
$\mathrm{C}(8)-\mathrm{C}(9)$ & $1.388(7)$ \\
$\mathrm{C}(9)-\mathrm{N}(2)$ & $1.370(7)$ \\
$\mathrm{N}(1)-\mathrm{Sb}(1)-\mathrm{N}(3)$ & $145.69(16)$ \\
$\mathrm{N}(1)-\mathrm{Sb}(1)-\mathrm{N}(2)$ & $72.78(15)$ \\
$\mathrm{N}(2)-\mathrm{Sb}(1)-\mathrm{N}(3)$ & $73.12(16)$ \\
$\mathrm{N}(1)-\mathrm{Sb}(1)-\mathrm{I}(1)$ & $90.56(12)$ \\
$\mathrm{N}(2)-\mathrm{Sb}(1)-\mathrm{I}(1)$ & $93.31(12)$ \\
$\mathrm{N}(3)-\mathrm{Sb}(1)-\mathrm{I}(1)$ & $87.71(15)$ \\
$\mathrm{O}(1)-\mathrm{Sb}(1)-\mathrm{I}(1)$ & $170.24(1)$
\end{tabular}




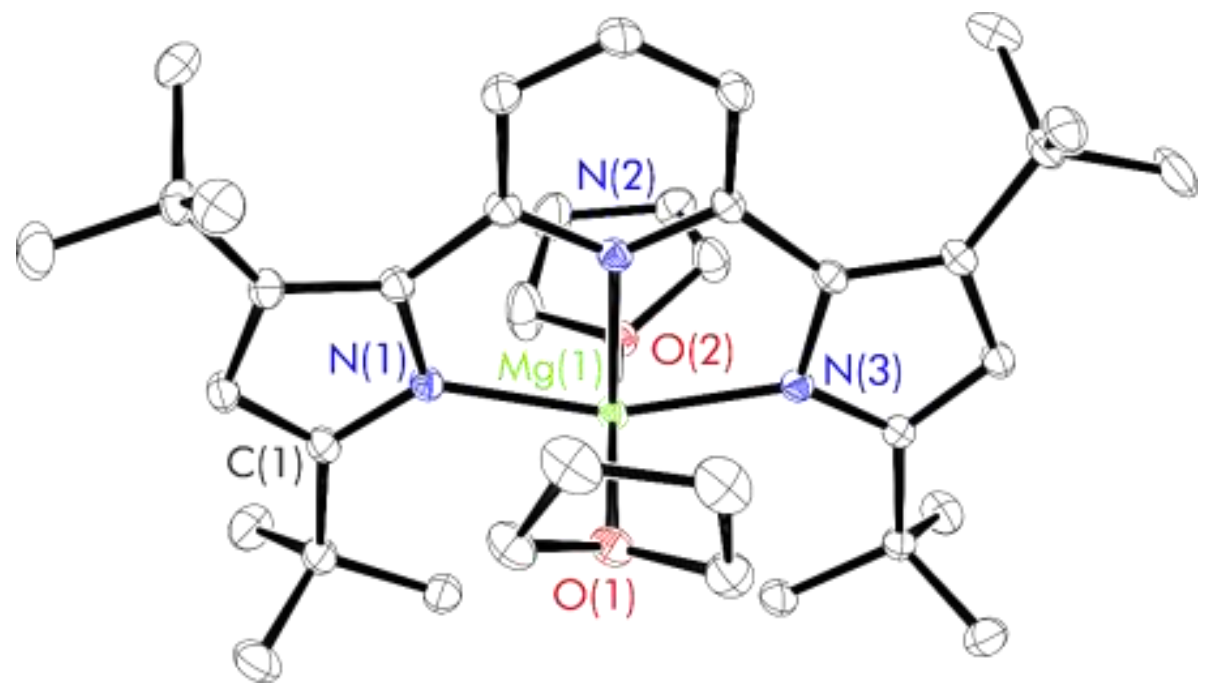

Figure S60: Thermal displacement ellipsoid drawing (30\% probability) of $\left.{ }^{\mathrm{t} B u}{ }^{\mathrm{B}} \mathrm{Bu} \mathrm{L}\right) \mathrm{Mg}(\mathrm{thf})_{2}(\mathbf{8})$ All hydrogen atoms have been omitted for clarity. Only one molecule from the asymmetric unit is depicted for clarity.

Table S13: Experimental metrical parameters (bond lengths in $\AA$ and angles in $\left.{ }^{\circ}\right)$ in $\left({ }^{\mathrm{t} B u},{ }^{\mathrm{B} u} \mathrm{~L}\right) \mathrm{Mg}(\operatorname{thf})_{2}$ (8). Values for only one molecule from the asymmetric unit are shown.

\begin{tabular}{cc}
\hline $\mathrm{Mg}(1)-\mathrm{N}(1)$ & $2.089(2)$ \\
$\mathrm{Mg}(1)-\mathrm{N}(2)$ & $2.0347(19)$ \\
$\mathrm{Mg}(1)-\mathrm{N}(3)$ & $2.074(2)$ \\
$\mathrm{Mg}(1)-\mathrm{O}(1)$ & $2.1835(19)$ \\
$\mathrm{Mg}(1)-\mathrm{O}(2)$ & $2.1696(18)$ \\
$\mathrm{N}(1)-\mathrm{Mg}(1)-\mathrm{N}(3)$ & $158.56(8)$ \\
$\mathrm{N}(1)-\mathrm{Mg}(1)-\mathrm{N}(2)$ & $79.09(8)$ \\
$\mathrm{N}(2)-\mathrm{Mg}(1)-\mathrm{N}(3)$ & $79.48(8)$ \\
$\mathrm{O}(1)-\mathrm{Mg}(1)-\mathrm{O}(2)$ & $163.51(7)$
\end{tabular}




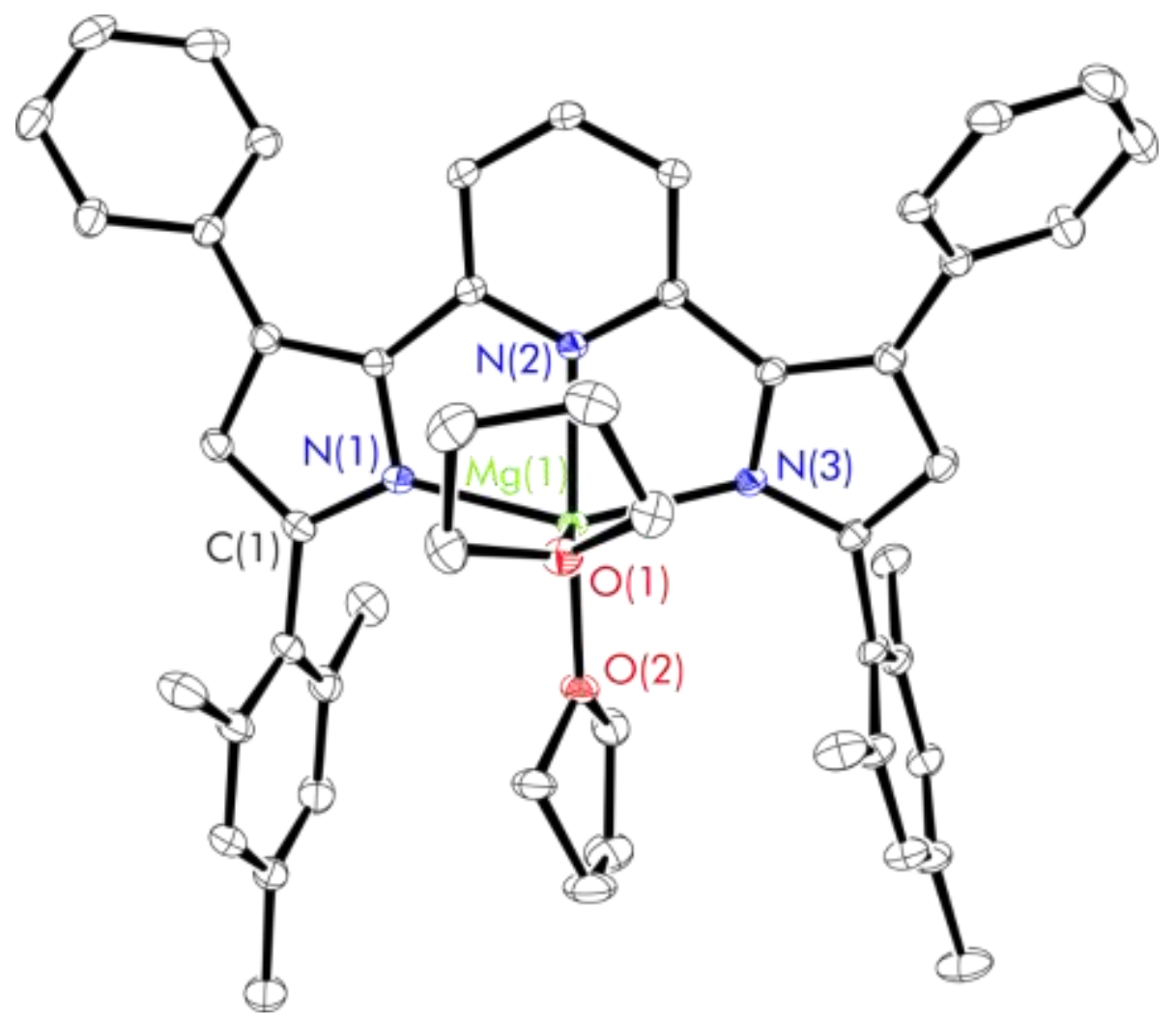

Figure S61: Thermal displacement ellipsoid drawing (30\% probability) of ( $\left.{ }^{\mathrm{Mes}, \mathrm{Ph}} \mathrm{L}\right) \mathrm{Mg}(\mathrm{thf})_{2}(\mathbf{1 0})$. All hydrogen atoms have been omitted for clarity.

Table S14: Experimental metrical parameters (bond lengths in $\AA$ and angles in $\left.{ }^{\circ}\right)$ in $\left({ }^{\mathrm{Mes}, \mathrm{Ph}} \mathrm{L}\right) \mathrm{Mg}(\mathrm{thf})_{2}$ (10).

\begin{tabular}{cc}
\hline $\mathrm{Mg}(1)-\mathrm{N}(1)$ & $2.1477(11)$ \\
$\mathrm{Mg}(1)-\mathrm{N}(2)$ & $2.0828(10)$ \\
$\mathrm{Mg}(1)-\mathrm{N}(3)$ & $2.1366(11)$ \\
$\mathrm{Mg}(1)-\mathrm{O}(1)$ & $2.1034(10)$ \\
$\mathrm{Mg}(1)-\mathrm{O}(2)$ & $2.0361(9)$ \\
$\mathrm{N}(1)-\mathrm{Mg}(1)-\mathrm{N}(3)$ & $149.33(4)$ \\
$\mathrm{N}(1)-\mathrm{Mg}(1)-\mathrm{N}(2)$ & $76.73(4)$ \\
$\mathrm{N}(2)-\mathrm{Mg}(1)-\mathrm{N}(3)$ & $76.74(4)$ \\
$\mathrm{O}(1)-\mathrm{Mg}(1)-\mathrm{O}(2)$ & $97.56(4)$
\end{tabular}




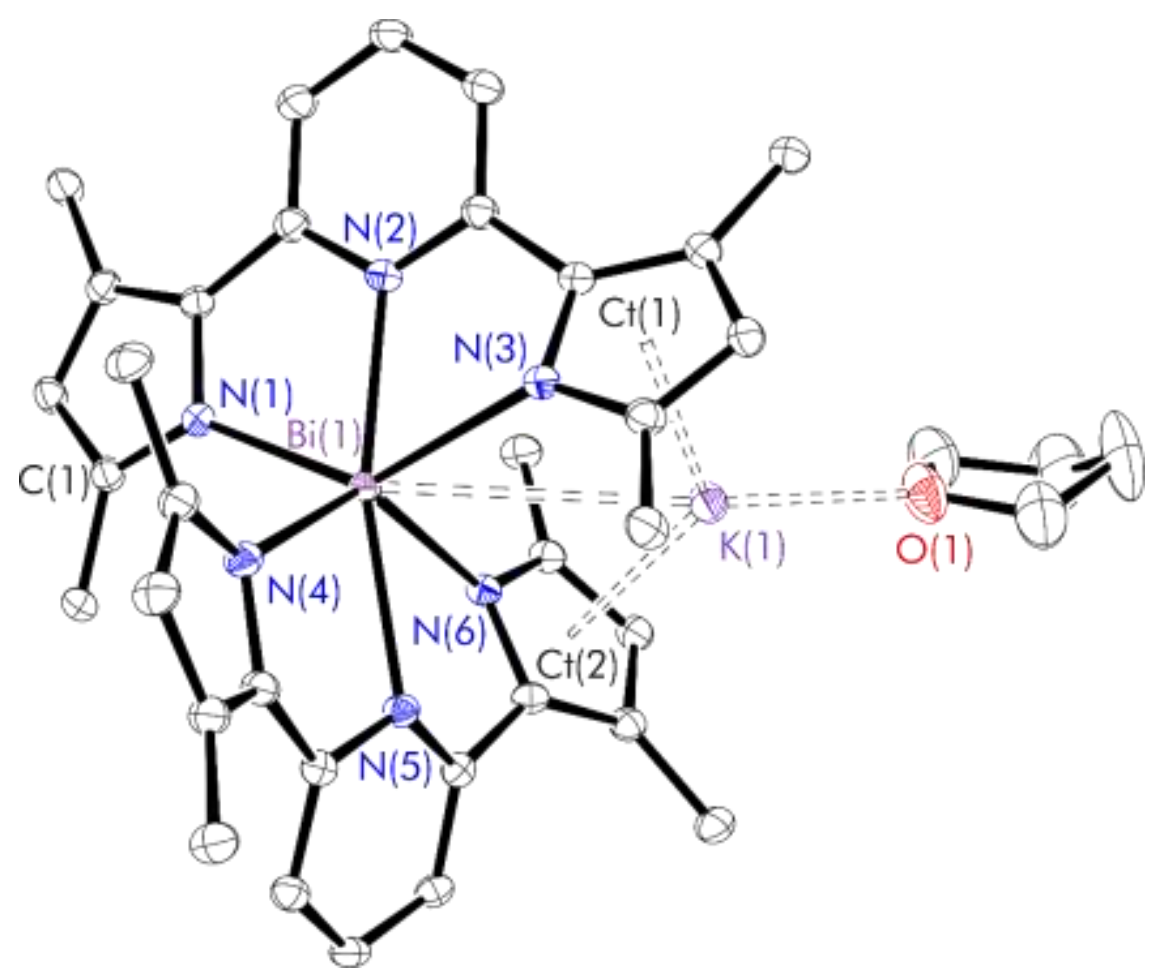

Figure S62: Thermal displacement ellipsoid drawing (30\% probability) of $\left.\mathrm{K}(\mathrm{thf})\left[{ }^{\mathrm{t} B u}{ }^{\mathrm{t} B u} \mathrm{~L}\right){ }_{2} \mathrm{Bi}\right](\mathbf{1 2})$. All hydrogen atoms have been omitted, ${ }^{\mathrm{B}} \mathrm{Bu}$ groups have been truncated and only one independent molecule in the asymmetric unit is shown for clarity.

Table S15: Experimental metrical parameters (bond lengths in $\AA$ and angles in ${ }^{\circ}$ ) in $\mathrm{K}($ thf $\left.)\left[{ }^{\mathrm{t} B u}{ }^{\mathrm{B}}{ }^{\mathrm{Bu}} \mathrm{L}\right)_{2} \mathrm{Bi}\right](\mathbf{1 2})$.

\begin{tabular}{cc}
\hline $\mathrm{Bi}(1)-\mathrm{K}(1)$ & $3.8957(8)$ \\
$\mathrm{Bi}(1)-\mathrm{N}(1)$ & $2.394(3)$ \\
$\mathrm{Bi}(1)-\mathrm{N}(2)$ & $2.429(3)$ \\
$\mathrm{Bi}(1)-\mathrm{N}(3)$ & $2.564(3)$ \\
$\mathrm{Bi}(1)-\mathrm{N}(4)$ & $2.393(3)$ \\
$\mathrm{Bi}(1)-\mathrm{N}(5)$ & $2.514(3)$ \\
$\mathrm{Bi}(1)-\mathrm{N}(6)$ & $2.668(3)$ \\
$\mathrm{K}(1)-\mathrm{O}(1)$ & $2.647(4)$ \\
$\mathrm{K}(1)-\mathrm{Ct}(1)$ & $2.8421(1)$ \\
$\mathrm{K}(1)-\mathrm{Ct}(2)$ & $2.8159(1)$ \\
$\mathrm{C}(5)-\mathrm{N}(2)$ & $1.362(5)$ \\
$\mathrm{C}(5)-\mathrm{C}(6)$ & $1.403(5)$ \\
$\mathrm{C}(6)-\mathrm{C}(7)$ & $1.376(6)$ \\
$\mathrm{C}(7)-\mathrm{C}(8)$ & $1.379(6)$ \\
$\mathrm{C}(8)-\mathrm{C}(9)$ & $1.395(5)$ \\
$\mathrm{C}(9)-\mathrm{N}(2)$ & $1.354(5)$ \\
$\mathrm{N}(1)-\mathrm{Bi}(1)-\mathrm{N}(3)$ & $135.74(10)$ \\
$\mathrm{N}(1)-\mathrm{Bi}(1)-\mathrm{N}(2)$ & $68.83(10)$ \\
$\mathrm{N}(2)-\mathrm{Bi}(1)-\mathrm{N}(3)$ & $68.57(10)$ \\
$\mathrm{N}(4)-\mathrm{Bi}(1)-\mathrm{N}(6)$ & $134.50(9)$ \\
$\mathrm{N}(4)-\mathrm{Bi}(1)-\mathrm{N}(5)$ & $69.20(10)$ \\
$\mathrm{N}(5)-\mathrm{Bi}(1)-\mathrm{N}(6)$ & $66.32(9)$
\end{tabular}




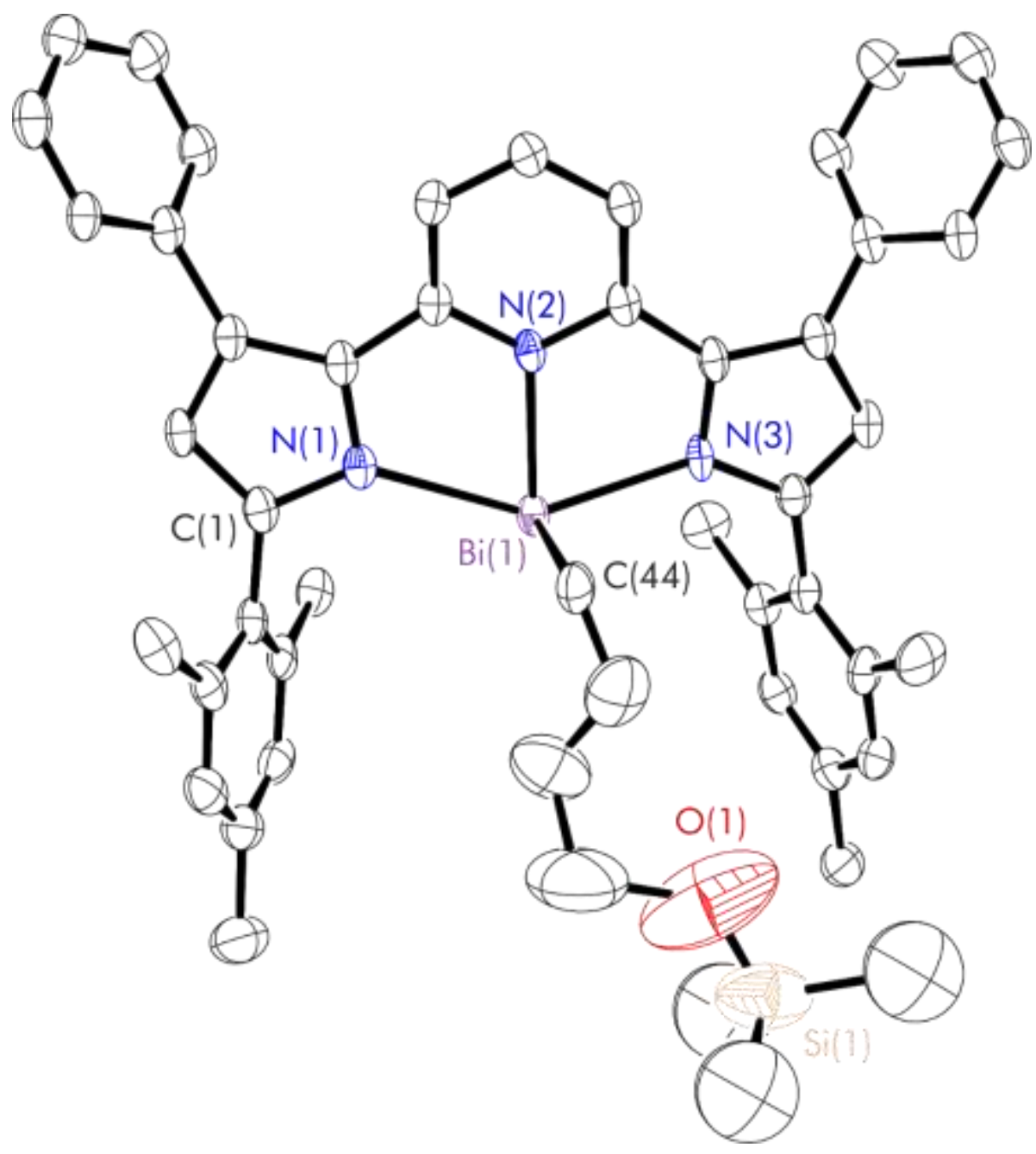

Figure S63: Thermal displacement ellipsoid drawing (30\% probability) of ( $\left.{ }^{\mathrm{Mes}, \mathrm{Ph}} \mathrm{L}\right) \mathrm{Bi}\left\{\left(\mathrm{CH}_{2}\right)_{4} \mathrm{OSiMe}_{3}\right\}$ (14). All hydrogen atoms have been omitted for clarity.

Table S16: Experimental metrical parameters (bond lengths in $\AA$ and angles in ${ }^{\circ}$ ) in ( $\left.{ }^{\mathrm{Mes}, \mathrm{Ph}} \mathrm{L}\right) \mathrm{Bi}\left\{\left(\mathrm{CH}_{2}\right)_{4} \mathrm{OSiMe}_{3}\right\}$ (14).

\begin{tabular}{cc}
\hline $\mathrm{Bi}(1)-\mathrm{N}(1)$ & $2.565(5)$ \\
$\mathrm{Bi}(1)-\mathrm{N}(2)$ & $2.311(5)$ \\
$\mathrm{Bi}(1)-\mathrm{N}(3)$ & $2.398(5)$ \\
$\mathrm{Bi}(1)-\mathrm{C}(44)$ & $2.175(8)$ \\
$\mathrm{C}(5)-\mathrm{N}(2)$ & $1.482(8)$ \\
$\mathrm{C}(5)-\mathrm{C}(6)$ & $1.398(9)$ \\
$\mathrm{C}(6)-\mathrm{C}(7)$ & $1.391(9)$ \\
$\mathrm{C}(7)-\mathrm{C}(8)$ & $1.495(10)$ \\
$\mathrm{C}(8)-\mathrm{C}(9)$ & $1.407(10)$ \\
$\mathrm{C}(9)-\mathrm{N}(2)$ & $1.398(8)$ \\
$\mathrm{N}(1)-\mathrm{Bi}(1)-\mathrm{N}(3)$ & $143.56(18)$ \\
$\mathrm{N}(1)-\mathrm{Bi}(1)-\mathrm{N}(2)$ & $67.55(17)$ \\
$\mathrm{N}(2)-\mathrm{Bi}(1)-\mathrm{N}(3)$ & $76.17(19)$ \\
$\mathrm{N}(1)-\mathrm{Bi}(1)-\mathrm{C}(44)$ & $90.2(2)$ \\
$\mathrm{N}(2)-\mathrm{Bi}(1)-\mathrm{C}(44)$ & $96.4(3)$ \\
$\mathrm{N}(3)-\mathrm{Bi}(1)-\mathrm{C}(44)$ & $90.5(2)$
\end{tabular}




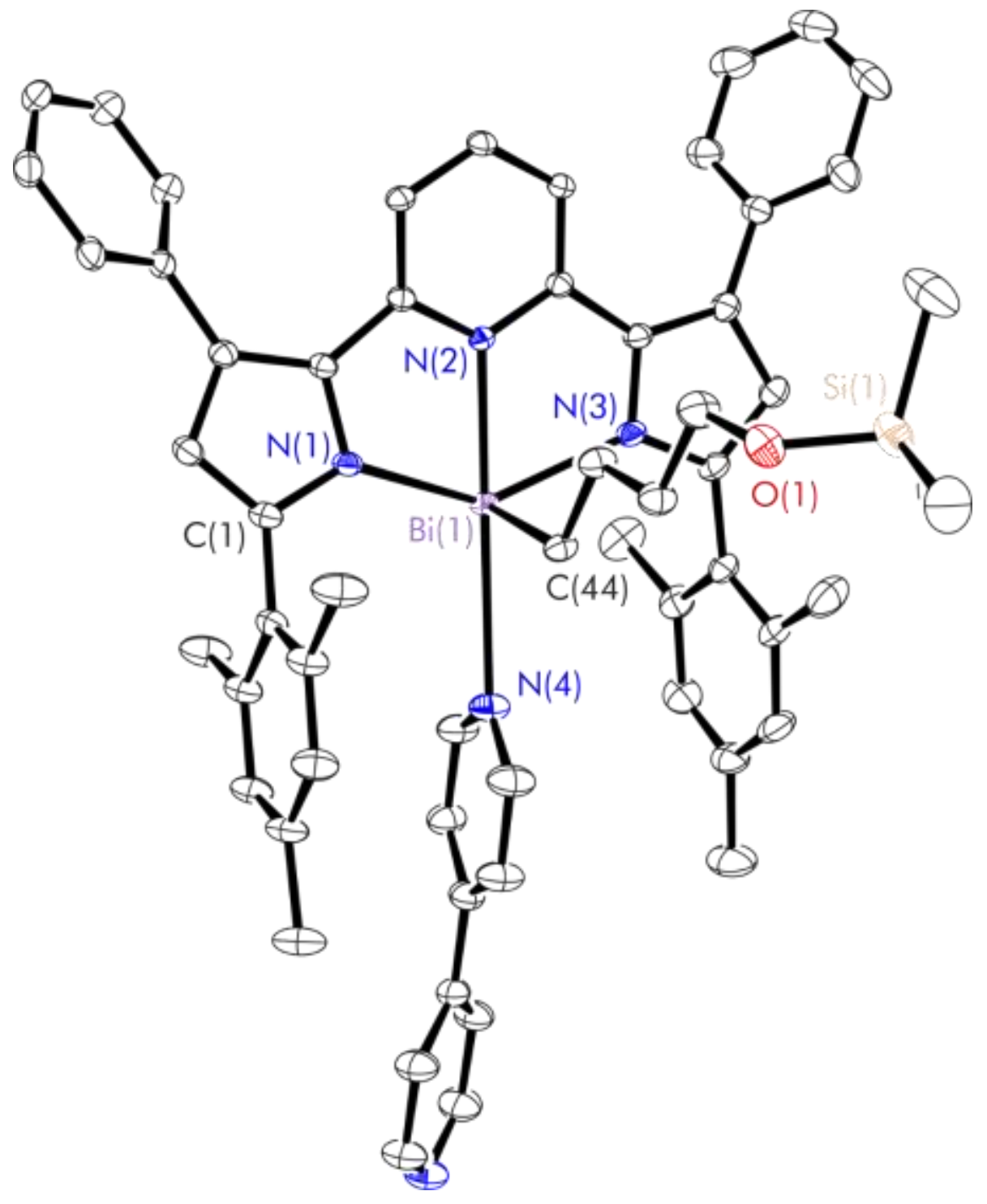

Figure S64: Thermal displacement ellipsoid drawing (30\% probability) of ( $\left.{ }^{\text {Mes,Ph }} \mathrm{L}\right) \mathrm{Bi}\left\{\left(\mathrm{CH}_{2}\right)_{4} \mathrm{OSiMe} 3\right\}\left(4,4^{\prime}\right.$-bipy) (14-4,4'-bipy). All hydrogen atoms have been omitted for clarity. 
Table S17: Experimental metrical parameters (bond lengths in $\AA$ and angles in ${ }^{\circ}$ ) in $\left({ }^{\mathrm{Mes}, \mathrm{Ph}} \mathrm{L}\right) \mathrm{Bi}\left\{\left(\mathrm{CH}_{2}\right)_{4} \mathrm{OSiMe}{ }_{3}\right\}(4,4$ '-bipy $)\left(\mathbf{1 4} \cdot \mathbf{4}, \mathbf{4}^{\prime}\right.$-'bipy $)$.

\begin{tabular}{cc}
\hline $\mathrm{Bi}(1)-\mathrm{N}(1)$ & $2.353(3)$ \\
$\mathrm{Bi}(1)-\mathrm{N}(2)$ & $2.325(3)$ \\
$\mathrm{Bi}(1)-\mathrm{N}(3)$ & $2.349(3)$ \\
$\mathrm{Bi}(1)-\mathrm{N}(4)$ & $2.8767(1)$ \\
$\mathrm{Bi}(1)-\mathrm{C}(44)$ & $2.218(4)$ \\
$\mathrm{C}(5)-\mathrm{N}(2)$ & $1.358(5)$ \\
$\mathrm{C}(5)-\mathrm{C}(6)$ & $1.393(5)$ \\
$\mathrm{C}(6)-\mathrm{C}(7)$ & $1.381(5)$ \\
$\mathrm{C}(7)-\mathrm{C}(8)$ & $1.380(5)$ \\
$\mathrm{C}(8)-\mathrm{C}(9)$ & $1.386(5)$ \\
$\mathrm{C}(9)-\mathrm{N}(2)$ & $1.364(5)$ \\
$\mathrm{C}(51)-\mathrm{C}(52)$ & $1.392(7)$ \\
$\mathrm{C}(52)-\mathrm{N}(4)$ & $1.330(6)$ \\
$\mathrm{C}(53)-\mathrm{N}(4)$ & $1.331(6)$ \\
$\mathrm{C}(53)-\mathrm{C}(54)$ & $1.384(6)$ \\
$\mathrm{C}(55)-\mathrm{C}(51)$ & $1.386(7)$ \\
$\mathrm{N}(1)-\mathrm{Bi}(1)-\mathrm{N}(3)$ & $137.36(11)$ \\
$\mathrm{N}(1)-\mathrm{Bi}(1)-\mathrm{N}(2)$ & $69.57(10)$ \\
$\mathrm{N}(2)-\mathrm{Bi}(1)-\mathrm{N}(3)$ & $69.72(11)$ \\
$\mathrm{N}(1)-\mathrm{Bi}(1)-\mathrm{C}(44)$ & $87.48(13)$ \\
$\mathrm{N}(2)-\mathrm{Bi}(1)-\mathrm{C}(44)$ & $96.31(13)$ \\
$\mathrm{N}(3)-\mathrm{Bi}(1)-\mathrm{C}(44)$ & $84.87(14)$ \\
&
\end{tabular}




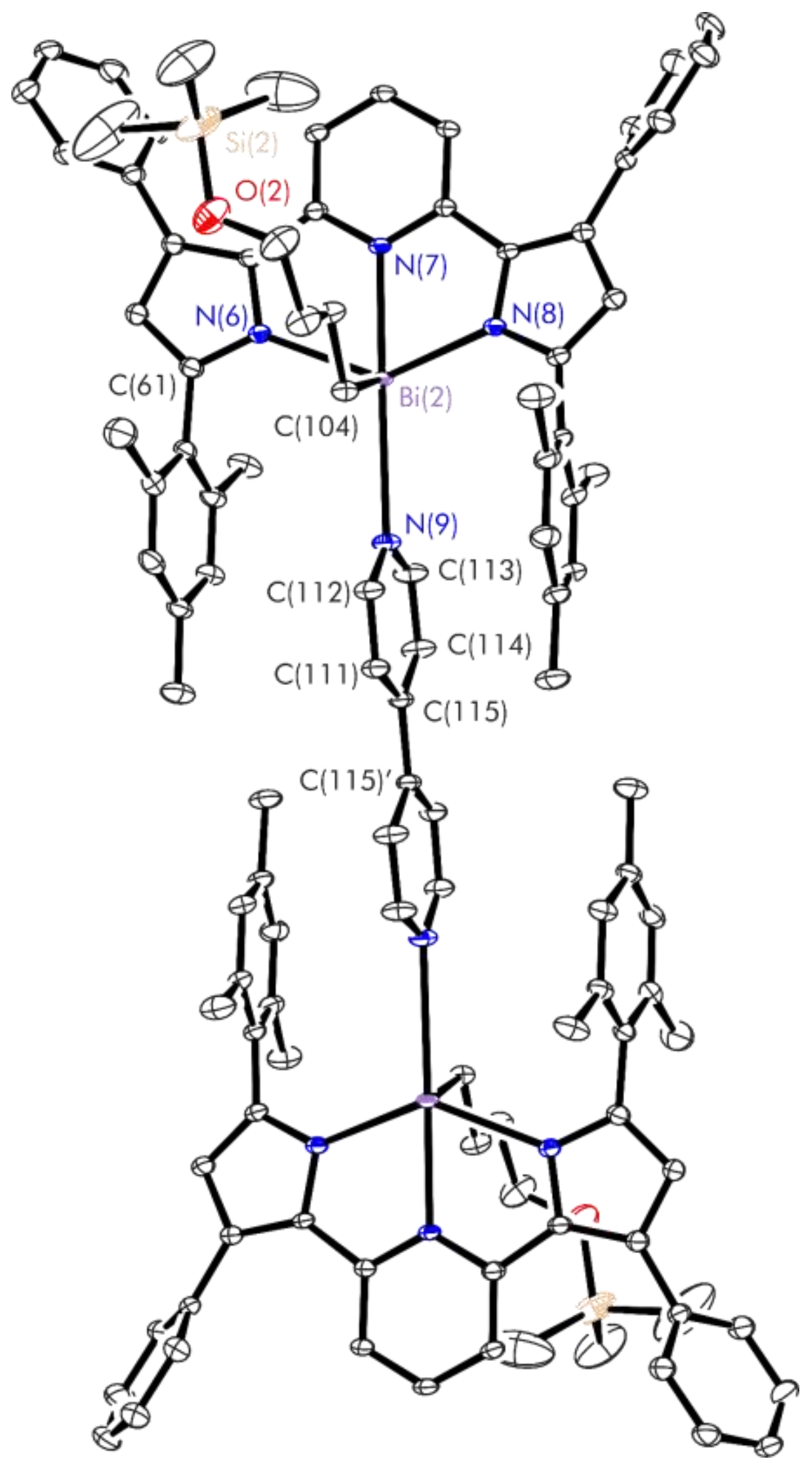

Figure S65: Thermal displacement ellipsoid drawing (30\% probability) of $\left[\left({ }^{\mathrm{Mes}, \mathrm{Ph}} \mathrm{L}\right) \mathrm{Bi}\left\{\left(\mathrm{CH}_{2}\right)_{4} \mathrm{OSiMe}{ }_{3}\right\}\right]_{2}(\boldsymbol{\mu}-4,4$ '-bipy $)\left(\mathbf{1 4}_{2}-\boldsymbol{\mu}-\mathbf{4}, \mathbf{4}\right.$ '-bipy $)$. All hydrogen atoms have been omitted for clarity. The full molecule has been generated by applying the symmetry operation $-\mathrm{x},-\mathrm{y}+1,-\mathrm{z}+1$. 
Table S18: Experimental metrical parameters (bond lengths in $\AA$ and angles in ${ }^{\circ}$ ) in $\left[\left({ }^{\mathrm{Mes}, \mathrm{Ph}} \mathrm{L}\right) \mathrm{Bi}\left\{\left(\mathrm{CH}_{2}\right)_{4} \mathrm{OSiMe}_{3}\right\}\right]_{2}(\boldsymbol{\mu}-4,4$ '-bipy $)\left(\mathbf{1 4}_{2}-\boldsymbol{\mu}-\mathbf{4}, \mathbf{4}\right.$ '-bipy $)$.

\begin{tabular}{cc}
\hline $\mathrm{Bi}(2)-\mathrm{N}(6)$ & $2.346(3)$ \\
$\mathrm{Bi}(2)-\mathrm{N}(7)$ & $2.350(3)$ \\
$\mathrm{Bi}(2)-\mathrm{N}(8)$ & $2.348(3)$ \\
$\mathrm{Bi}(2)-\mathrm{N}(9)$ & $2.9034(1)$ \\
$\mathrm{Bi}(2)-\mathrm{C}(104)$ & $2.224(4)$ \\
$\mathrm{C}(65)-\mathrm{N}(7)$ & $1.366(5)$ \\
$\mathrm{C}(65)-\mathrm{C}(66)$ & $1.401(5)$ \\
$\mathrm{C}(66)-\mathrm{C}(67)$ & $1.381(6)$ \\
$\mathrm{C}(67)-\mathrm{C}(68)$ & $1.377(6)$ \\
$\mathrm{C}(68)-\mathrm{C}(69)$ & $1.403(5)$ \\
$\mathrm{C}(69)-\mathrm{N}(7)$ & $1.353(5)$ \\
$\mathrm{C}(111)-\mathrm{C}(112)$ & $1.387(5)$ \\
$\mathrm{C}(112)-\mathrm{N}(9)$ & $1.330(6)$ \\
$\mathrm{N}(9)-\mathrm{C}(113)$ & $1.327(6)$ \\
$\mathrm{C}(113)-\mathrm{C}(114)$ & $1.384(6)$ \\
$\mathrm{C}(114)-\mathrm{C}(115)$ & $1.390(6)$ \\
$\mathrm{C}(115)-\mathrm{C}(111)$ & $1.393(6)$
\end{tabular}




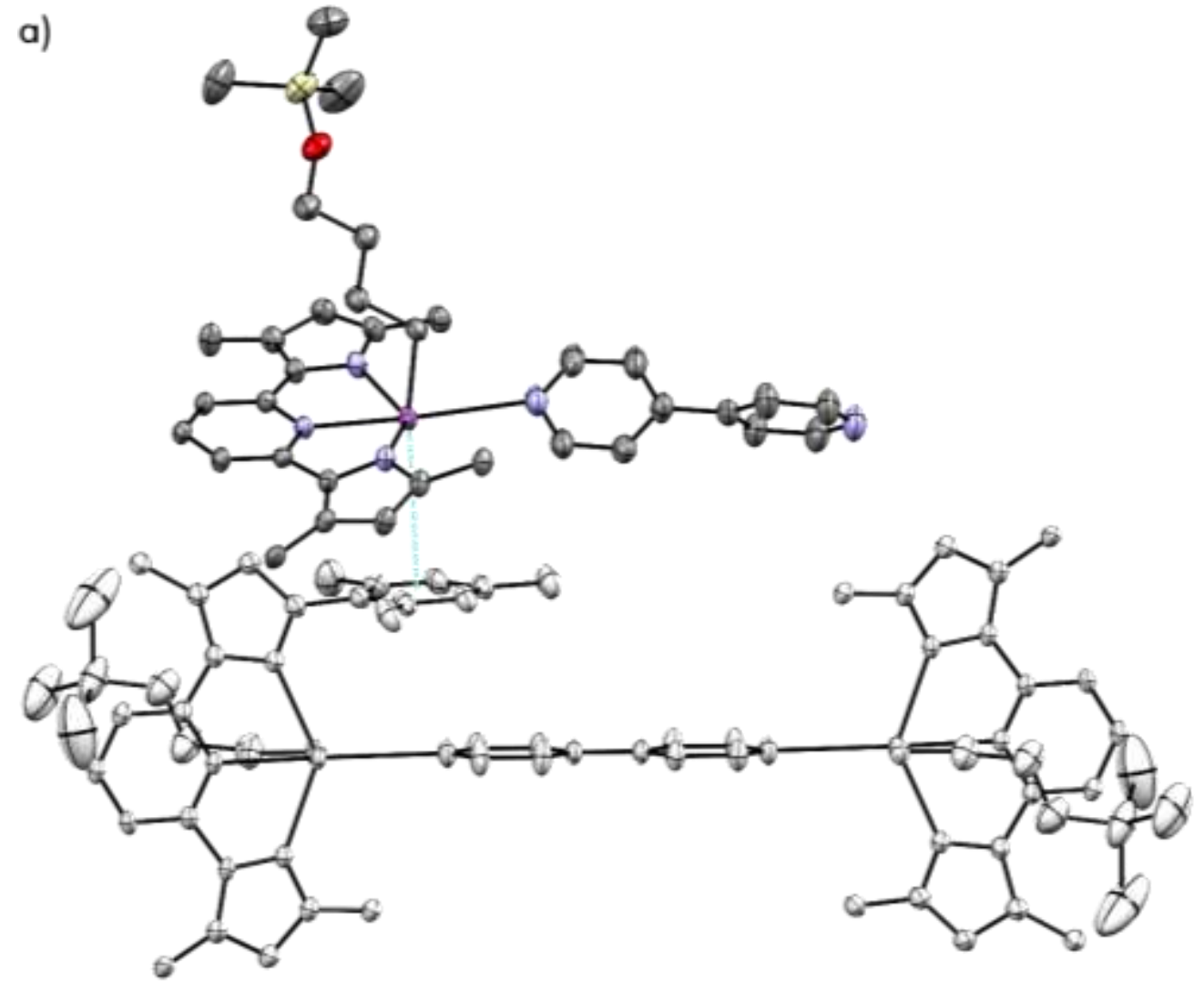

b)

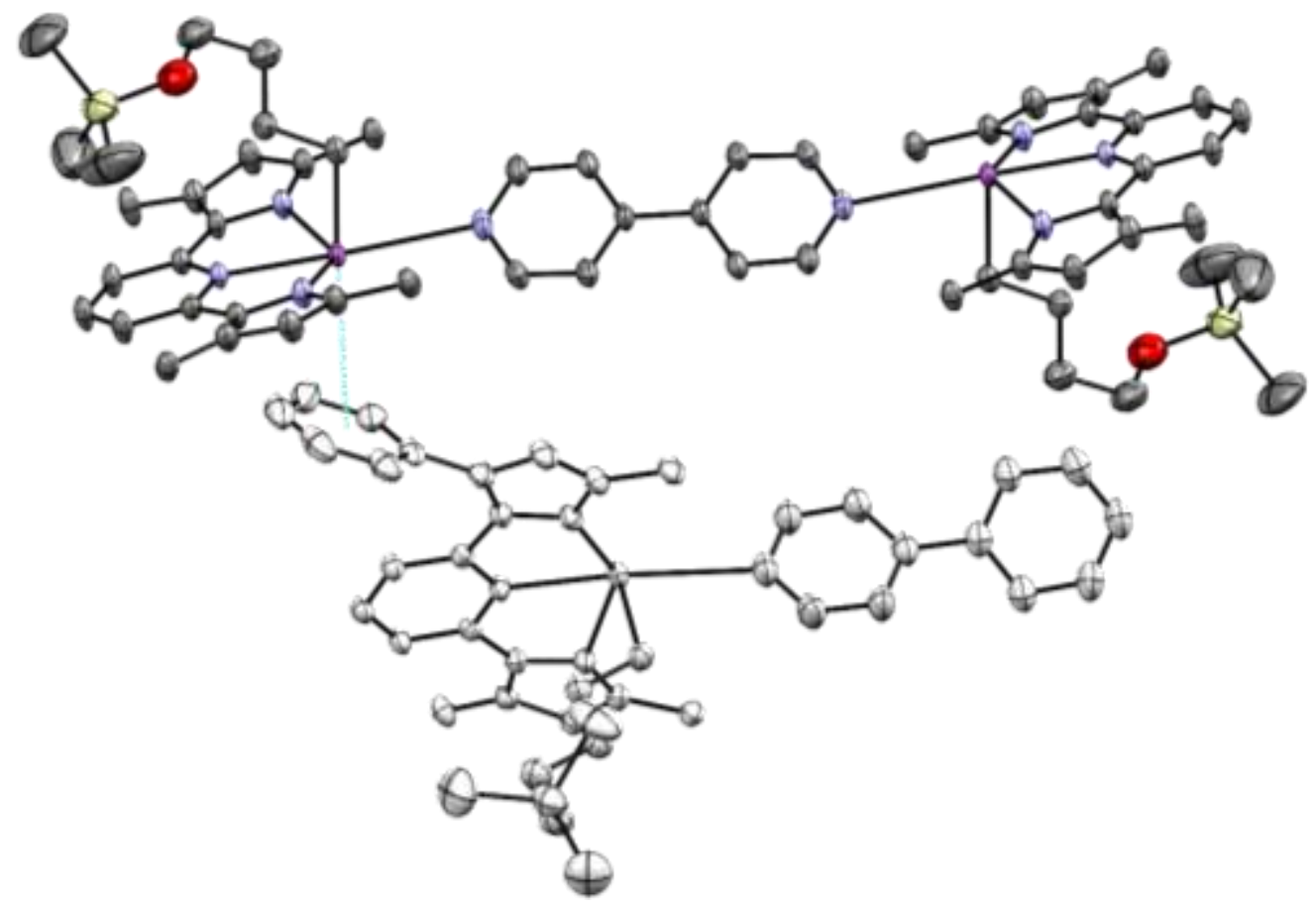

Figure S66: Representations of the co-crystallised ( $\left.{ }^{\mathrm{Mes}, \mathrm{Ph}} \mathrm{L}\right) \mathrm{Bi}\left\{\left(\mathrm{CH}_{2}\right)_{4} \mathrm{OSiMe}_{3}\right\}(4,4$ '-bipy) and $\left[\left({ }^{\text {Mes, }, \mathrm{Ph}} \mathrm{L}\right) \mathrm{Bi}\left\{\left(\mathrm{CH}_{2}\right)_{4} \mathrm{OSiMe}{ }_{3}\right\}\right]_{2}\left(4,4^{\prime}\right.$-bipy $)$ units, showing with a dashed cyan line that the position trans to the alkyl chain in both a) monomer and b) dimer is shielded by an aryl group of the adjacent dimer and monomer respectively. 


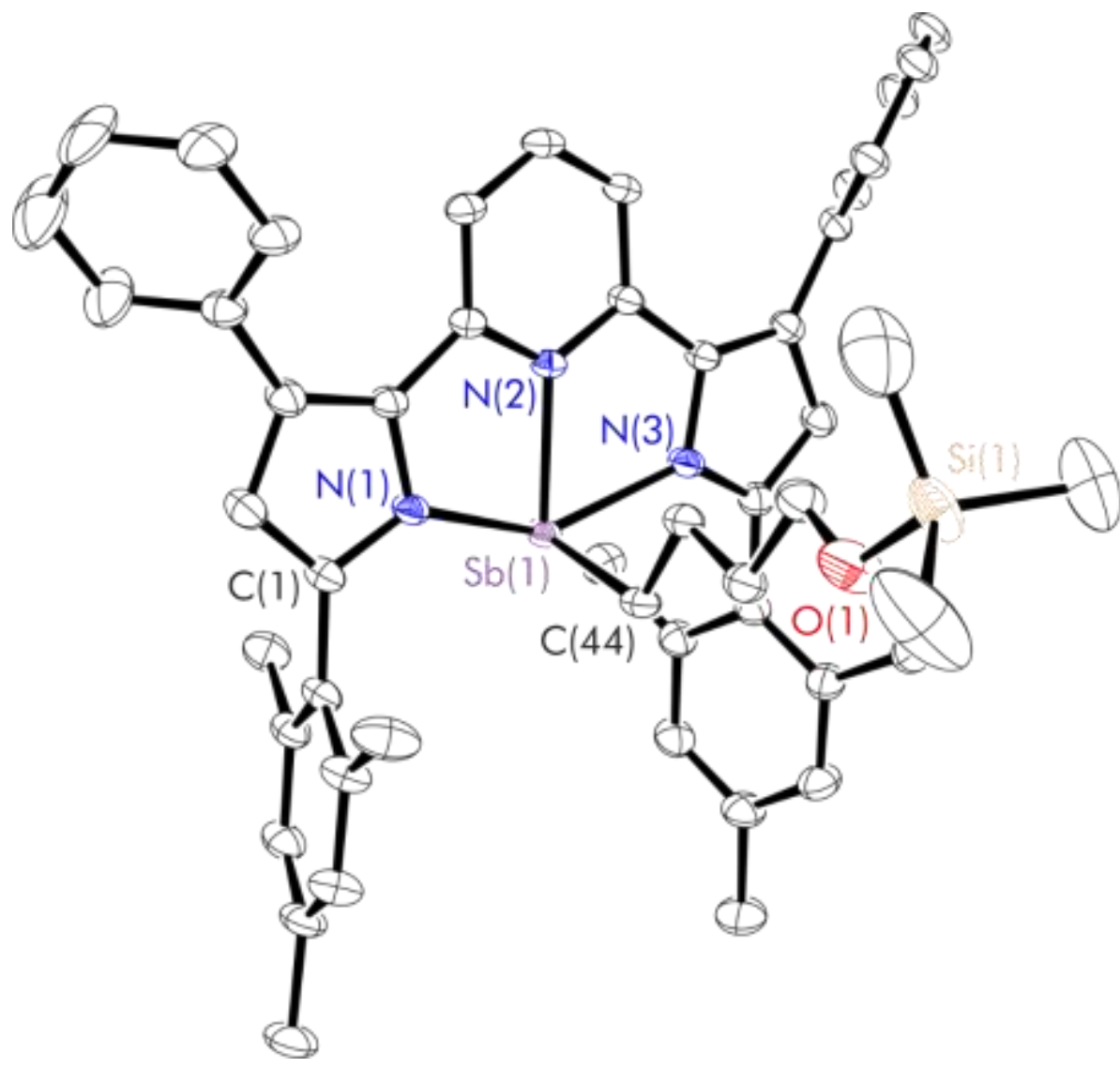

Figure S67: Thermal displacement ellipsoid drawings (30\% probability) of ( $\left.{ }^{\mathrm{Mes}, \mathrm{Ph}} \mathrm{L}\right) \mathrm{Sb}\left\{\left(\mathrm{CH}_{2}\right)_{4} \mathrm{OSiMe}_{3}\right\}$ (15). All hydrogen atoms have been omitted for clarity.

Table S19: Experimental metrical parameters (bond lengths in $\AA$ and angles in ${ }^{\circ}$ ) in $\left({ }^{\mathrm{Mes}, \mathrm{Ph}} \mathrm{L}\right) \mathrm{Sb}\left\{\left(\mathrm{CH}_{2}\right)_{4} \mathrm{OSiMe}{ }_{3}\right\}(\mathbf{1 5})$.

\begin{tabular}{cc}
\hline $\mathrm{Sb}(1)-\mathrm{N}(1)$ & $2.2070(18)$ \\
$\mathrm{Sb}(1)-\mathrm{N}(2)$ & $2.1820(17)$ \\
$\mathrm{Sb}(1)-\mathrm{N}(3)$ & $2.2023(17)$ \\
$\mathrm{Sb}(1)-\mathrm{C}(44)$ & $2.139(2)$ \\
$\mathrm{C}(5)-\mathrm{N}(2)$ & $1.368(3)$ \\
$\mathrm{C}(5)-\mathrm{C}(6)$ & $1.398(3)$ \\
$\mathrm{C}(6)-\mathrm{C}(7)$ & $1.384(3)$ \\
$\mathrm{C}(7)-\mathrm{C}(8)$ & $1.377(3)$ \\
$\mathrm{C}(8)-\mathrm{C}(9)$ & $1.395(3)$ \\
$\mathrm{C}(9)-\mathrm{N}(2)$ & $1.370(3)$ \\
$\mathrm{N}(1)-\mathrm{Sb}(1)-\mathrm{N}(3)$ & $144.38(6)$ \\
$\mathrm{N}(1)-\mathrm{Sb}(1)-\mathrm{N}(2)$ & $72.76(6)$ \\
$\mathrm{N}(2)-\mathrm{Sb}(1)-\mathrm{N}(3)$ & $72.52(6)$ \\
$\mathrm{N}(1)-\mathrm{Sb}(1)-\mathrm{C}(44)$ & $87.55(8)$ \\
$\mathrm{N}(2)-\mathrm{Sb}(1)-\mathrm{C}(44)$ & $98.63(7)$ \\
$\mathrm{N}(3)-\mathrm{Sb}(1)-\mathrm{C}(44)$ & $89.98(8)$
\end{tabular}




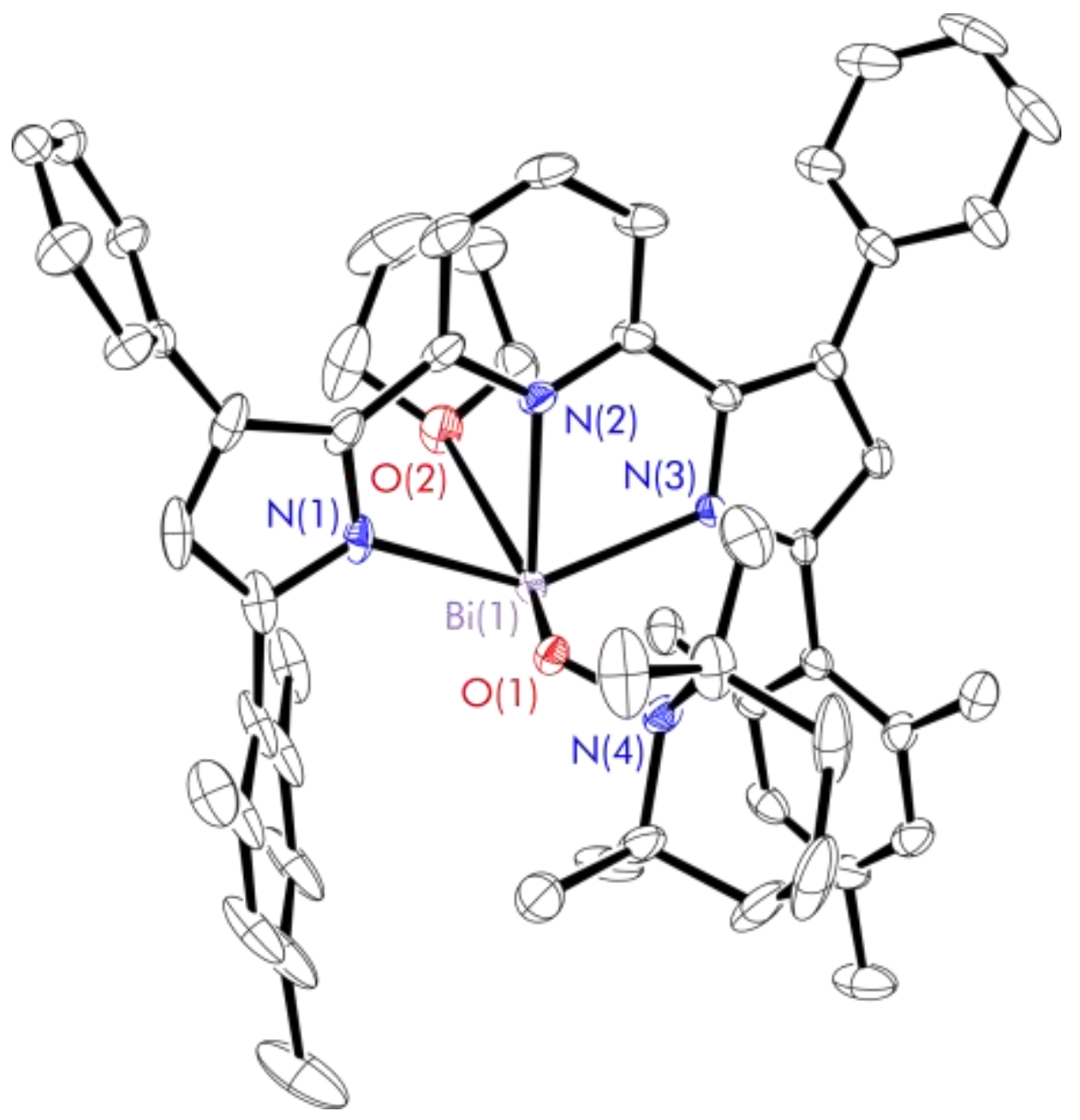

Figure S68: Thermal displacement ellipsoid drawings (30\% probability) of ( $\left.{ }^{\mathrm{Mes}, \mathrm{Ph}} \mathrm{L}\right) \mathrm{Bi}(\mathrm{OTEMP})(\mathrm{thf})$ (16·thf). All hydrogen atoms have been omitted for clarity.

Table S20: Experimental metrical parameters (bond lengths in $\AA$ and angles in ${ }^{\circ}$ ) in $\left({ }^{\mathrm{Mes}, \mathrm{Ph}} \mathrm{L}\right) \mathrm{Bi}(\mathrm{OTEMP})($ thf $)(\mathbf{1 6} \cdot$ thf $)$.

\begin{tabular}{cc}
\hline $\mathrm{Bi}(1)-\mathrm{N}(1)$ & $2.269(3)$ \\
$\mathrm{Bi}(1)-\mathrm{N}(2)$ & $2.314(3)$ \\
$\mathrm{Bi}(1)-\mathrm{N}(3)$ & $2.342(3)$ \\
$\mathrm{Bi}(1)-\mathrm{O}(1)$ & $2.086(2)$ \\
$\mathrm{Bi}(1)-\mathrm{O}(2)$ & $3.0919(1)$ \\
$\mathrm{C}(5)-\mathrm{N}(2)$ & $1.369(4)$ \\
$\mathrm{C}(5)-\mathrm{C}(6)$ & $1.390(6)$ \\
$\mathrm{C}(6)-\mathrm{C}(7)$ & $1.377(7)$ \\
$\mathrm{C}(7)-\mathrm{C}(8)$ & $1.380(6)$ \\
$\mathrm{C}(8)-\mathrm{C}(9)$ & $1.407(5)$ \\
$\mathrm{C}(9)-\mathrm{N}(2)$ & $1.347(5)$ \\
$\mathrm{N}(1)-\mathrm{Bi}(1)-\mathrm{N}(3)$ & $140.31(11)$ \\
$\mathrm{N}(1)-\mathrm{Bi}(1)-\mathrm{N}(2)$ & $70.39(11)$ \\
$\mathrm{N}(2)-\mathrm{Bi}(1)-\mathrm{N}(3)$ & $69.96(10)$ \\
$\mathrm{N}(1)-\mathrm{Bi}(1)-\mathrm{O}(1)$ & $78.93(10)$ \\
$\mathrm{N}(2)-\mathrm{Bi}(1)-\mathrm{O}(1)$ & $92.65(10)$ \\
$\mathrm{N}(3)-\mathrm{Bi}(1)-\mathrm{O}(1)$ & $100.94(10)$
\end{tabular}




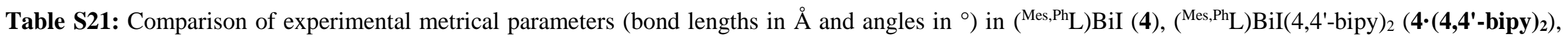
$\left\{\left({ }^{\mathrm{Mes}, \mathrm{Ph}} \mathrm{L}\right) \mathrm{Bi}\right\}_{2}(\mu-\mathrm{Br}) \mathrm{Br}\left(\mathrm{Et}_{2} \mathrm{O}\right)\left(\mathbf{5}_{2} \cdot \mathbf{E t}_{2} \mathbf{O}\right),\left\{\left({ }^{\mathrm{Mes}, \mathrm{Ph}} \mathrm{L}\right) \mathrm{Bi}\right\}_{2}(\mu-\mathrm{Cl}) \mathrm{Cl}(\mathrm{thf})\left(\mathbf{6}_{2} \cdot \mathbf{t h f}\right),\left({ }^{\mathrm{Mes}, \mathrm{Ph}} \mathrm{L}\right) \mathrm{SbI}(\mathbf{7})$ and $\left({ }^{\mathrm{Mes}, \mathrm{Ph}} \mathrm{L}\right) \mathrm{SbI}\left(\mathrm{Et}_{2} \mathrm{O}\right)\left(\mathbf{7} \cdot \mathbf{E t}_{2} \mathbf{O}\right)$.

\begin{tabular}{|c|c|c|c|c|c|c|}
\hline & 4 & $4 \cdot\left(4,4^{\prime}-\text { bipy }\right)_{2}$ & $5_{2} \cdot \mathbf{E t}_{2} \mathrm{O}$ & $66_{2} \cdot$ thf & 7 & $7 \cdot \mathrm{Et}_{2} \mathrm{O}$ \\
\hline $\mathrm{M}(1)-\mathrm{X}(1)$ & $2.8128(2)$ & $2.8824(2)$ & $2.6363(5)$ & $2.494(2)$ & $2.7413(3)$ & $2.7742(5)$ \\
\hline $\mathrm{M}(1)-\mathrm{X}(2)$ & - & - & $3.3236(1)$ & $3.1729(1)$ & - & \\
\hline $\mathrm{M}(2)-\mathrm{X}(2)$ & - & - & $2.6976(6)$ & $2.584(3)$ & - & \\
\hline $\mathrm{M}(1)-\mathrm{N}(1)$ & $2.3018(19)$ & $2.3204(19)$ & $2.294(3)$ & $2.274(6)$ & $2.216(2)$ & $2.217(4)$ \\
\hline $\mathrm{M}(1)-\mathrm{N}(2)$ & $2.3017(19)$ & $2.3257(19)$ & $2.301(3)$ & $2.302(5)$ & $2.177(2)$ & $2.179(4)$ \\
\hline $\mathrm{M}(1)-\mathrm{N}(3)$ & $2.3039(19)$ & $2.3343(19)$ & $2.281(3)$ & $2.279(5)$ & $2.207(3)$ & $2.208(4)$ \\
\hline $\mathrm{M}(1 / 2)-\mathrm{O}(1)$ & - & - & $2.705(6)$ & $2.553(10)$ & - & $2.9146(1)$ \\
\hline $\mathrm{M}(2)-\mathrm{N}(4)$ & - & $2.9096(1)^{\mathrm{a}}$ & $2.294(5)$ & $2.295(8)$ & - & \\
\hline $\mathrm{M}(2)-\mathrm{N}(5)$ & - & - & $2.297(4)$ & $2.279(7)$ & - & \\
\hline $\mathrm{M}(2)-\mathrm{N}(6)$ & - & $2.769(2)$ & $2.300(4)$ & $2.301(7)$ & - & \\
\hline $\mathrm{C}(5)-\mathrm{N}(2)$ & $1.362(3)$ & $1.365(3)$ & $1.366(5)$ & $1.364(9)$ & $1.370(4)$ & $1.355(7)$ \\
\hline$C(5)-C(6)$ & $1.397(3)$ & $1.399(3)$ & $1.400(6)$ & $1.411(9)$ & $1.398(4)$ & $1.403(7)$ \\
\hline$C(6)-C(7)$ & $1.383(3)$ & $1.387(4)$ & $1.381(6)$ & $1.371(10)$ & $1.380(4)$ & $1.379(7)$ \\
\hline$C(7)-C(8)$ & $1.386(3)$ & $1.381(3)$ & $1.395(6)$ & $1.387(11)$ & $1.393(4)$ & $1.383(8)$ \\
\hline $\mathrm{C}(8)-\mathrm{C}(9)$ & $1.395(3)$ & $1.404(3)$ & $1.400(6)$ & $1.392(10)$ & $1.390(4)$ & $1.388(7)$ \\
\hline $\mathrm{C}(9)-\mathrm{N}(2)$ & $1.365(3)$ & $1.359(3)$ & $1.366(5)$ & $1.373(9)$ & $1.369(4)$ & $1.370(7)$ \\
\hline $\mathrm{X}(1)-\mathrm{M}(1)-\mathrm{X}(2)$ & - & & $171.89(1)$ & $175.38(1)$ & - & \\
\hline $\mathrm{X}(2)-\mathrm{M}(2)-\mathrm{O}(1)$ & - & & $176.87(13)$ & $175.9(2)$ & - & \\
\hline $\mathrm{N}(1)-\mathrm{M}(1)-\mathrm{N}(3)$ & $140.78(7)$ & $139.89(7)$ & $139.92(12)$ & $139.7(2)$ & $145.45(9)$ & $145.69(16)$ \\
\hline $\mathrm{N}(1)-\mathrm{M}(1)-\mathrm{N}(2)$ & $70.67(7)$ & $69.96(7)$ & $70.11(12)$ & $70.0(2)$ & 73.07(9) & $72.78(15)$ \\
\hline $\mathrm{N}(2)-\mathrm{M}(1)-\mathrm{N}(3)$ & $70.20(7)$ & $70.08(7)$ & $69.97(12)$ & $70.0(2)$ & $72.81(9)$ & $73.12(16)$ \\
\hline $\mathrm{N}(1)-\mathrm{Bi}(1)-\mathrm{X}(1)$ & $92.55(5)$ & $87.61(5)$ & $90.56(9)$ & $88.09(16)$ & $91.14(6)$ & $90.56(12)$ \\
\hline $\mathrm{N}(2)-\mathrm{Bi}(1)-\mathrm{X}(1)$ & $99.93(5)$ & $91.26(5)$ & $92.33(9)$ & $88.91(16)$ & $101.11(6)$ & $93.31(12)$ \\
\hline $\mathrm{N}(3)-\mathrm{Bi}(1)-\mathrm{X}(1)$ & $91.65(5)$ & $89.93(5)$ & 87.62(9) & $92.19(16)$ & $90.17(7)$ & $87.71(15)$ \\
\hline $\mathrm{N}(4)-\mathrm{Bi}(2)-\mathrm{N}(6)$ & - & - & $140.50(15)$ & $139.9(3)$ & - & - \\
\hline $\mathrm{N}(4)-\mathrm{Bi}(2)-\mathrm{N}(5)$ & - & - & $70.32(15)$ & $70.6(3)$ & - & - \\
\hline $\mathrm{N}(5)-\mathrm{Bi}(2)-\mathrm{N}(6)$ & - & - & 70.61(16) & $70.4(3)$ & - & - \\
\hline
\end{tabular}


Table S22: Comparison of experimental metrical parameters (bond lengths in $\AA$ and angles in $\left.{ }^{\circ}\right)$ in $\left({ }^{\mathrm{Mes}, \mathrm{Ph}} \mathrm{L}\right) \mathrm{BiI}(\mathbf{4}),\left({ }^{\mathrm{Mes}, \mathrm{Ph}} \mathrm{L}\right) \mathrm{SbI}(\mathbf{7}),\left({ }^{\mathrm{Mes}, \mathrm{Ph}} \mathrm{L}\right) \mathrm{Bi}\left\{\left(\mathrm{CH}_{2}\right)_{4} \mathrm{OSiMe}{ }_{3}\right\}$

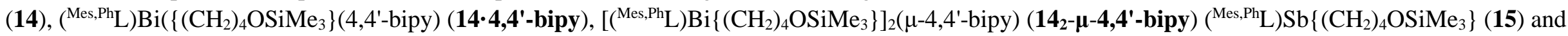
(Mes,Ph $\mathrm{L}) \mathrm{Bi}(\mathrm{OTEMP})(\mathrm{thf})(\mathbf{1 6} \cdot \mathbf{t h f})$.

\begin{tabular}{|c|c|c|c|c|c|c|c|}
\hline & 4 & 7 & 14 & 14·4,4'-bipy & 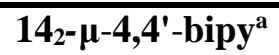 & 15 & $16 \cdot$ thf \\
\hline $\mathrm{M}(1)-\mathrm{X}(1)$ & $2.8128(2)$ & $2.7413(3)$ & - & - & & & \\
\hline $\mathrm{M}(1)-\mathrm{C}(44)$ & & & $2.175(8)$ & $2.218(4)$ & $2.224(4)^{\mathrm{a}}$ & $2.139(2)$ & \\
\hline $\mathrm{M}(1)-\mathrm{N}(1)$ & $2.3018(19)$ & $2.216(2)$ & $2.565(5)$ & $2.353(3)$ & $2.346(3)$ & $2.2070(18)$ & $2.269(3)$ \\
\hline $\mathrm{M}(1)-\mathrm{N}(2)$ & $2.3017(19)$ & $2.177(2)$ & $2.311(5)$ & $2.325(3)$ & $2.350(3)$ & $2.1820(17)$ & $2.314(3)$ \\
\hline $\mathrm{M}(1)-\mathrm{N}(3)$ & $2.3039(19)$ & $2.207(3)$ & $2.398(5)$ & $2.349(3)$ & $2.348(3)$ & $2.2023(17)$ & $2.342(3)$ \\
\hline $\mathrm{M}(1)-\mathrm{N}(4)$ & - & - & - & $2.8767(1)$ & $2.9034(1)$ & - & - \\
\hline $\mathrm{M}(1)-\mathrm{O}(1)$ & - & - & - & - & - & - & $2.086(2)$ \\
\hline $\mathrm{M}(1)-\mathrm{O}(2)$ & - & - & - & - & - & - & $3.0919(1)$ \\
\hline $\mathrm{C}(5)-\mathrm{N}(2)$ & $1.362(3)$ & $1.370(4)$ & $1.482(8)$ & $1.358(5)$ & $1.366(5)$ & $1.368(3)$ & $1.369(4)$ \\
\hline$C(5)-C(6)$ & $1.397(3)$ & $1.398(4)$ & $1.398(9)$ & $1.393(5)$ & $1.401(5)$ & $1.398(3)$ & $1.390(6)$ \\
\hline$C(6)-C(7)$ & $1.383(3)$ & $1.380(4)$ & $1.391(9)$ & $1.381(5)$ & $1.381(6)$ & $1.384(3)$ & $1.377(7)$ \\
\hline$C(7)-C(8)$ & $1.386(3)$ & $1.393(4)$ & $1.495(10)$ & $1.380(5)$ & $1.377(6)$ & $1.377(3)$ & $1.380(6)$ \\
\hline $\mathrm{C}(8)-\mathrm{C}(9)$ & $1.395(3)$ & $1.390(4)$ & $1.407(10)$ & $1.386(5)$ & $1.403(5)$ & $1.395(3)$ & $1.407(5)$ \\
\hline $\mathrm{C}(9)-\mathrm{N}(2)$ & $1.365(3)$ & $1.369(4)$ & $1.398(8)$ & $1.364(5)$ & $1.353(5)$ & $1.370(3)$ & $1.347(5)$ \\
\hline $\mathrm{N}(1)-\mathrm{M}(1)-\mathrm{N}(3)$ & $140.78(7)$ & $145.45(9)$ & $143.56(18)$ & & $135.46(11)$ & $144.38(6)$ & $140.31(11)$ \\
\hline $\mathrm{N}(1)-\mathrm{M}(1)-\mathrm{N}(2)$ & $70.67(7)$ & 73.07(9) & $67.55(17)$ & & $68.73(11)$ & $72.76(6)$ & $70.39(11)$ \\
\hline $\mathrm{N}(2)-\mathrm{M}(1)-\mathrm{N}(3)$ & $70.20(7)$ & $72.81(9)$ & $76.17(19)$ & & $69.23(11)$ & $72.52(6)$ & $69.96(10)$ \\
\hline $\mathrm{N}(1)-\mathrm{M}(1)-\mathrm{X}(1)$ & $92.55(5)$ & $91.14(6)$ & - & - & - & - & - \\
\hline $\mathrm{N}(2)-\mathrm{M}(1)-\mathrm{X}(1)$ & $99.93(5)$ & 101.11(6) & - & - & - & - & - \\
\hline $\mathrm{N}(3)-\mathrm{M}(1)-\mathrm{X}(1)$ & $91.65(5)$ & $90.17(7)$ & - & - & - & - & - \\
\hline $\mathrm{N}(1)-\mathrm{M}(1)-\mathrm{C}(44)$ & - & - & $90.2(2)$ & $87.48(13)$ & $86.16(14)$ & $87.55(8)$ & - \\
\hline $\mathrm{N}(2)-\mathrm{M}(1)-\mathrm{C}(44)$ & - & - & $96.4(3)$ & $96.31(13)$ & $98.21(12)$ & $98.63(7)$ & - \\
\hline $\mathrm{N}(3)-\mathrm{M}(1)-\mathrm{C}(44)$ & - & - & $90.5(2)$ & $84.87(14)$ & $85.86(14)$ & $89.98(8)$ & - \\
\hline
\end{tabular}

${ }^{a}$ See Figure S65 and Table S18 for true atom labels. Equivalent distances and angles have been represented here for the sake of comparison. 


\section{Electrochemical data}

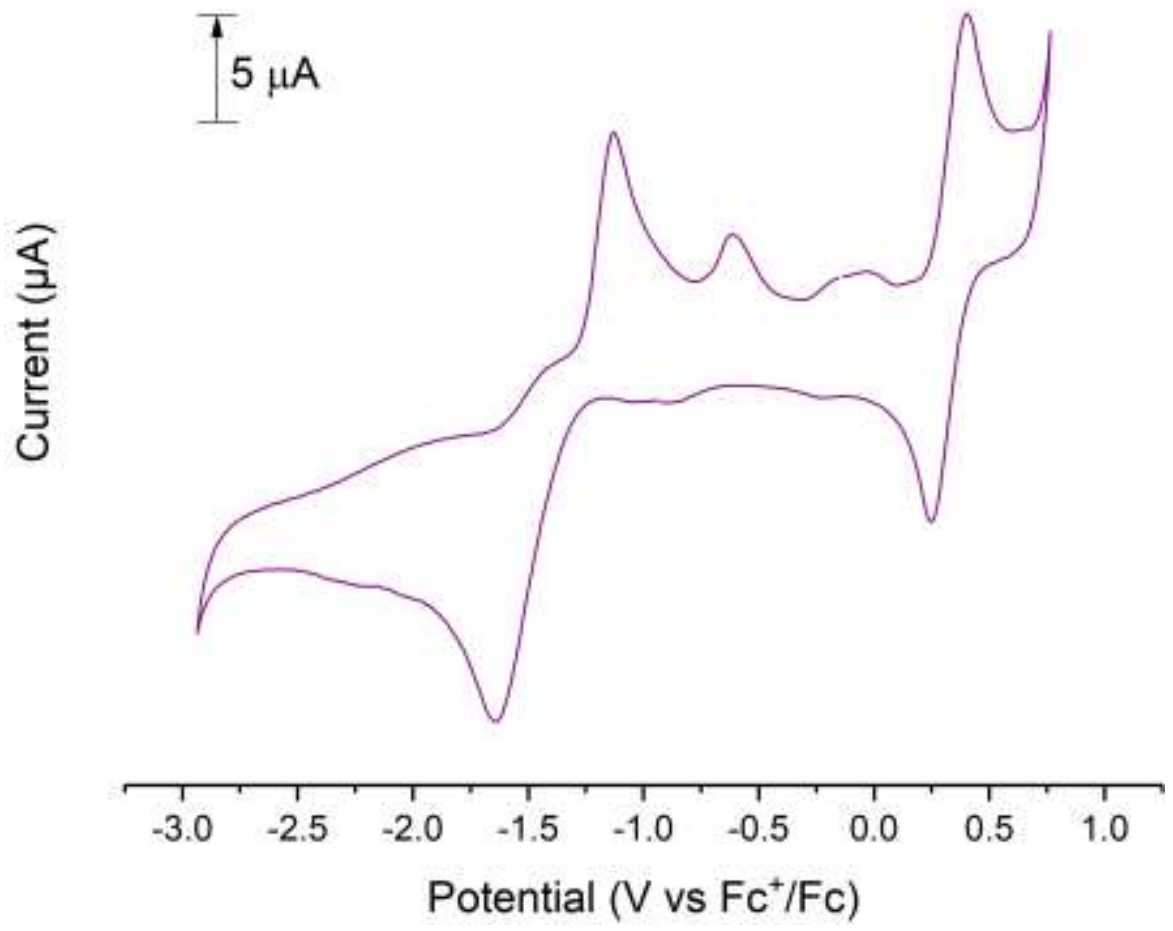

Figure S69: Cyclic voltammogram of $\left({ }^{\mathrm{Mes}, \mathrm{Ph}} \mathrm{L}\right) \mathrm{BiI}(\mathrm{thf})_{2}\left(\mathbf{4} \cdot \mathbf{t h f}_{\mathbf{2}}\right)$ in thf scanned in the range $-2.9-0.8 \mathrm{~V}$ (glassy carbon working electrode, $0.1 \mathrm{M}\left[{ }^{\mathrm{n}} \mathrm{Bu}_{4} \mathrm{~N}\right]\left[\mathrm{PF}_{6}\right]$, scan rate $100 \mathrm{mVs}^{-1}, 298 \mathrm{~K}$ ).

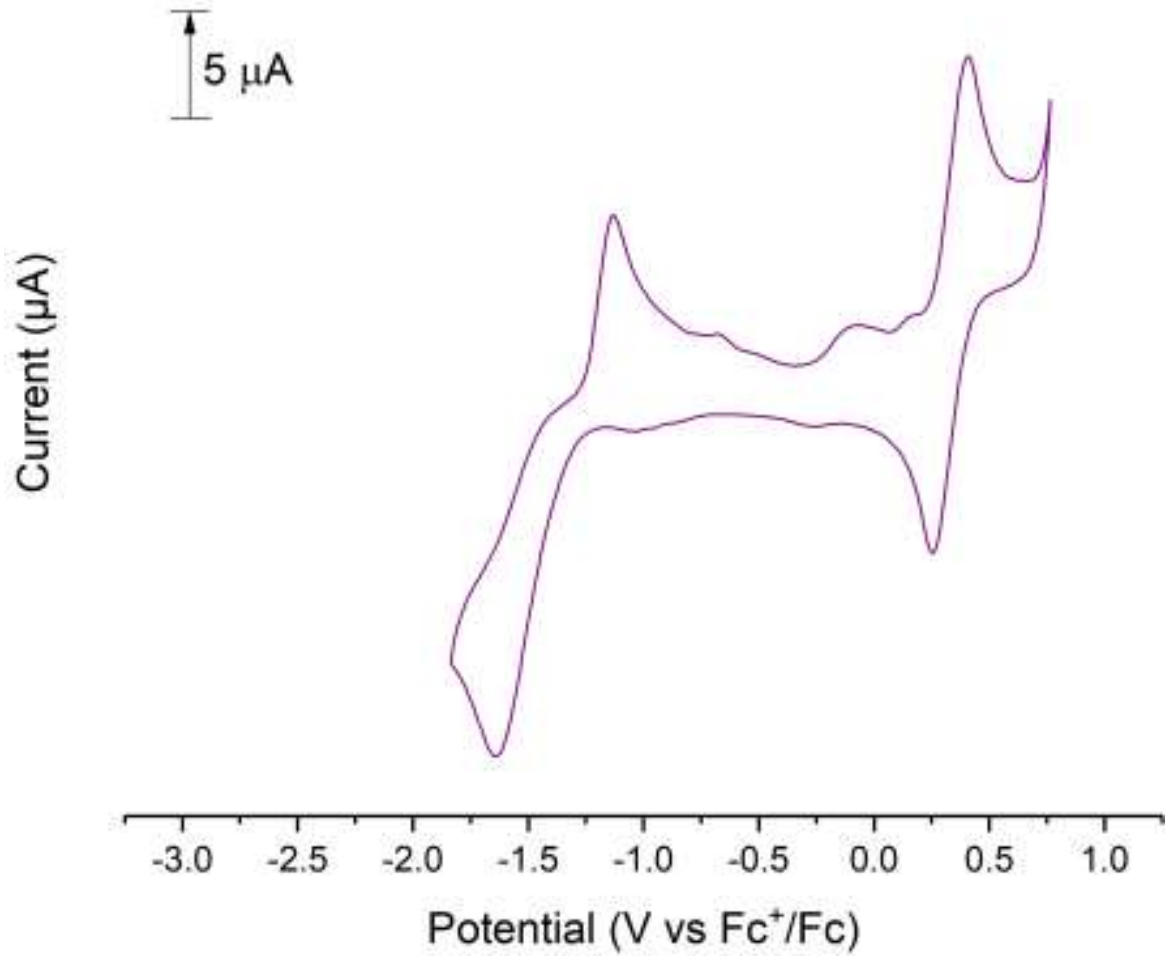

Figure S70: Cyclic voltammogram of $\left({ }^{\mathrm{Mes}, \mathrm{Ph}} \mathrm{L}\right) \mathrm{BiI}(\mathrm{thf})_{2}\left(\mathbf{4} \cdot \mathbf{t h f} \mathbf{f}_{2}\right)$ in thf scanned in the range $-1.8-0.8 \mathrm{~V}$ (glassy carbon working electrode, $0.1 \mathrm{M}\left[{ }^{\mathrm{n}} \mathrm{Bu}_{4} \mathrm{~N}\right]\left[\mathrm{PF}_{6}\right]$, scan rate $100 \mathrm{mVs}^{-1}, 298 \mathrm{~K}$ ). 


\section{References}

1. Abebayehu, A.; Dutta, R.; Lee, C.-H., Synthesis, Characterization and Properties of Expanded Pyriporphyrins: A New Family of Alkylidenyl Porphyrin Homologues Bearing meso-Exocyclic Double Bonds. Chem. Eur. J. 2016, 22 (39), 13850-13856.

2. Kaim, W., Effects of cyclic $8 \pi$-electron conjugation in reductively silylated nitrogen heterocycles. J. Am. Chem. Soc. 1983, 105 (4), 707-713.

3. Bonyhady, S. J.; Jones, C.; Nembenna, S.; Stasch, A.; Edwards, A. J.; McIntyre, G. J., $\beta-$ Diketiminate-Stabilized Magnesium(I) Dimers and Magnesium(II) Hydride Complexes: Synthesis, Characterization, Adduct Formation, and Reactivity Studies. Chem. Eur. J. 2010, 16 (3), 938-955.

4. Zhang, Y.; Petersen, J. L.; Milsmann, C., A Luminescent Zirconium(IV) Complex as a Molecular Photosensitizer for Visible Light Photoredox Catalysis. J. Am. Chem. Soc. 2016, 138 (40), 13115-13118.

5. Klappa, J. J.; Rich, A. E.; McNeill, K., One-Step Synthesis of 3,5-Disubstituted-2-pyridylpyrroles from the Condensation of 1,3-Diones and 2-(Aminomethyl)pyridine. Org. Lett. 2002, 4 (3), 435-437.

6. $\quad$ Alan Jones, R.; Karatza, M.; Voro, T. N.; Civeir, P. U.; Franck, A.; Ozturk, O.; Seaman, J. P.; Whitmore, A. P.; Williamson, D. J., Extended heterocyclic systems 1. The synthesis and characterisation of pyrrolylpyridines, alternating pyrrole: Pyridine oligomers and polymers, and related systems. Tetrahedron 1996, 52 (26), 8707-8724.

7. Hakey, B. M.; Darmon, J. M.; Zhang, Y.; Petersen, J. L.; Milsmann, C., Synthesis and Electronic Structure of Neutral Square-Planar High-Spin Iron(II) Complexes Supported by a Dianionic Pincer Ligand. Inorg. Chem. 2019, 58 (2), 1252-1266.

8. Cosier, J.; Glazer, A. M., A nitrogen-gas-stream cryostat for general X-ray diffraction studies. J. Appl. Crystallogr. 1986, 19 (2), 105-107.

9. CrysAlisPRO, Oxford Diffraction /Agilent Technologies UK Ltd, Yarnton, England.

10. Altomare, A.; Cascarano, G.; Giacovazzo, C.; Guagliardi, A., Completion and refinement of crystal structures with SIR92. J. Appl. Crystallogr. 1993, 26 (3), 343-350.

11. Palatinus, L.; Chapuis, G., SUPERFLIP - a computer program for the solution of crystal structures by charge flipping in arbitrary dimensions. J. Appl. Crystallogr. 2007, 40 (4), 786-790.

12. Farrugia, L., WinGX suite for small-molecule single-crystal crystallography. J. Appl. Crystallogr. 1999, $32(4), 837-838$. 